\title{
Summary Information and Data Sets for the HBCU Solar Measurements Network
}

William Marion

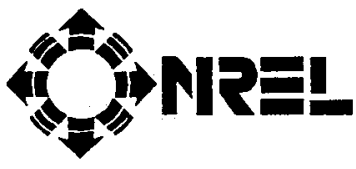

National Renewable Energy Laboratory 1617 Cole Boulevard Golden, Colorado 80401-3393

A national laboratory of the U.S. Department of Energy Managed by Midwest Research Institute under contract No. DE-AC36-83CH10093

Prepared under Tașk No. RA411040

August 1994 


\section{NOTICE}

This report was prepared as an account of work sponsored by an agency of the United States government. Neither the United States government nor any agency thereof, nor any of their employees, makes any warranty, express or implied, or assumes any legal liability or responsibility for the accuracy, completeness. or usefulness of any information, apparatus, product, or process disclosed, or represents that its use would not infringe privately owned rights. Reference herein to any specific commercial product, process, or service by trade name, trademark, manufacturer, or otherwise does not necessarily constitute or imply its endorsement, recommendation, or favoring by the United States government or any agency thereof. The views and opinions of authors expressed herein do not necessarily state or reflect those of the United States government or any agency thereof.

Available to DOE and DOE contractors from:

Office of Scientific and Technical Information (OSTI)

P.O. Box 62

Oak Ridge, TN 37831

Prices available by calling (615) 576-8401

Available to the public from:

National Technical Information Service (NTIS)

U.S. Department of Commerce

5285 Port Royal Road

Springfield, VA 22161

(703) 487-4650 


\section{Preface}

This work was performed under the National Renewable Energy Laboratory's (NREL's) Solar Radiation Resource Assessment Project Task No. RA411040. It provides summary information and describes hourly data sets for solar radiation elements measured from 1985 to 1993 by the Historically Black Colleges and Universities (HBCU) solar measurements network.

The HBCU stations are maintained and operated by university faculty and students, whose efforts made this report possible. These participants include Dr. Theodore Nicholson, Lucius Mims, Fred Arku, Donald Bullen, Martin Kinyungu, and Derrick Jackson from Bethune-Cookman College; Dr. C. Lewis Foster, Richard Lackey, Roger Cole, and Lee Haye from Bluefield State College; Dr. Sultana Khan, Eric Overton, Pam Faber, Eddie Vinson, and Roland Wiborg from Elizabeth City State University; Dr. J. Singh from Mississippi Valley State University; Dr. Tom C. Whitney and James Malloy from South Carolina State University; and Kendall W. Hill from Savannah State College.

NREL initiated the network to provide better regional coverage of the solar resource and to comply with President Reagan's Executive Order directing all federal agencies to implement programs to strengthen the nation's HBCUs. NREL staff involved in the network include Tom Stoffel (project manager and technical monitor), Gene Maxwell (conceptual designer), Martin Rymes (data processing software developer), Steve Wilcox (data acquisition and processing software developer), Toula Ismailidis (data manager), and Brian Rieper (contract administrator). The author would also like to acknowledge NREL staff members Carol Riordan, Dave Renné, and Tom Stoffel for their contributions to this report and their review.

\section{Approved for the National Renewable Energy Laboratory}

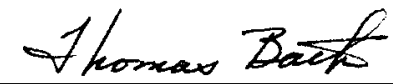

Thomas Bath, Director

Analytic Studies Division

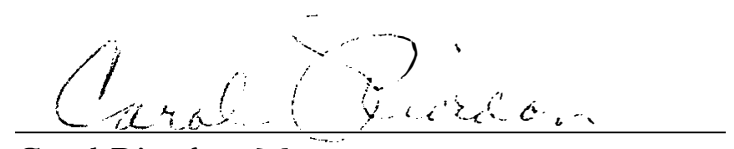

Carol Riordan, Manager

Technology \& Resource Assessment Branch

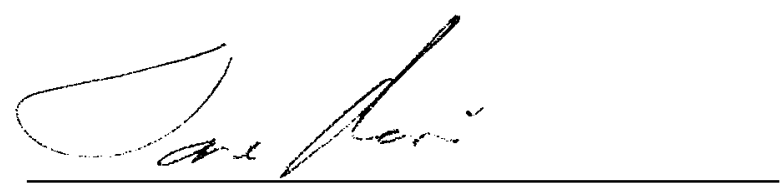

Dave Renné, Project Manager

Solar Radiation Resource Assessment Project 


\section{Table of Contents}

$\underline{\text { Section }} \quad \underline{\text { Page }}$

Preface $\quad \ldots \ldots \ldots \ldots \ldots \ldots \ldots \ldots \ldots \ldots \ldots \ldots \ldots \ldots \ldots \ldots \ldots \ldots$ iii

$1.0 \quad$ Introduction $\ldots \ldots \ldots \ldots \ldots \ldots \ldots \ldots \ldots \ldots \ldots \ldots \ldots \ldots$

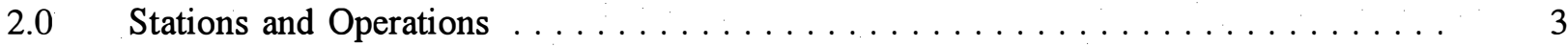

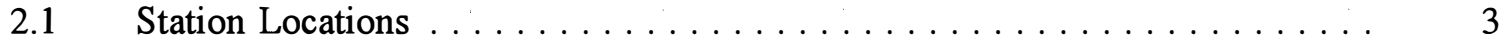

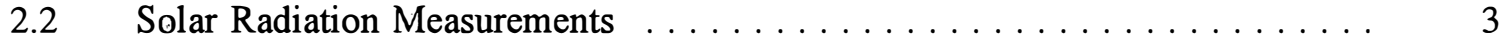

2.3 Maintenance and Instrument Calibration $\ldots \ldots \ldots \ldots \ldots \ldots \ldots \ldots$

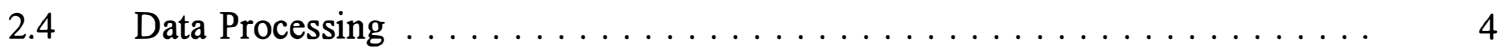

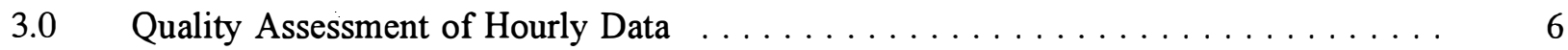

3.1 Quality Assessment for Solar Radiation Data . . . . . . . . . . . . . . . . 6

$4.0 \quad$ Hourly Data Files $\ldots \ldots \ldots \ldots \ldots \ldots \ldots \ldots \ldots \ldots \ldots \ldots \ldots \ldots$

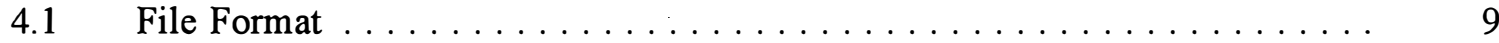

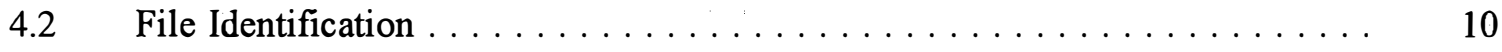

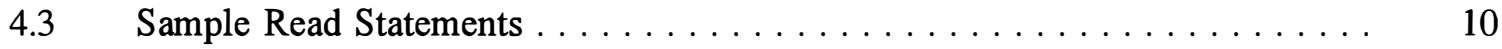

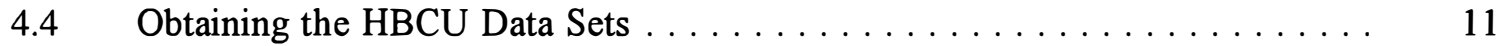

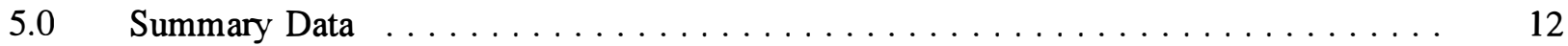

$5.1 \quad$ Missing Data and Quality Assessed Data Summaries . . . . . . . . . . . 12

5.2 Influence of Missing Data on Summary Data Uncertainties . . . . . . . . . . . 13

$5.3 \quad$ Average Daily Radiation . . . . . . . . . . . . . . . . . . . . . . . . . . . 15

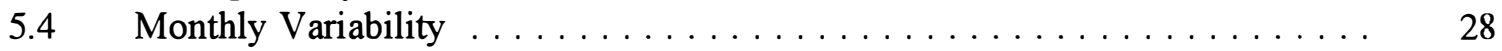

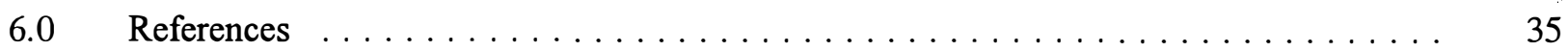

Appendix $\quad$ Instrument and Quality Assessment Summaries $\ldots \ldots \ldots \ldots \ldots \ldots$ 


\section{List of Figures}

Figure

Page

1-1. The six-station HBCU network provides additional coverage between measurement stations operated by the National Weather Service for the National Oceanic and

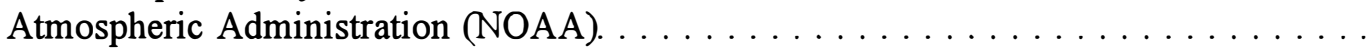

2-1 Student Eddie Vinson examines the instrument-mounting platform for measuring diffuse horizontal, global horizontal, and direct normal radiation at Elizabeth City State University . . . . . . . . . . . . . . . . . . . . . . . .

2-2 Log used to record maintenance of instruments and data acquisition system . . . . . 5

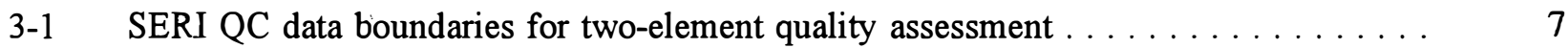

4-1 Data for Mississippi Valley State University for July 4, $1985 \ldots \ldots \ldots \ldots$

5-1 Monthly variability of global horizontal radiation for Bethune-Cookman College . . . 29

5-2 Monthly variability of direct normal radiation for Bethune-Cookman College . . . . . 29

5-3 Monthly variability of global horizontal radiation for Bluefield State College . . . . 30

5-4 Monthly variability of direct normal radiation for Bluefield State College 30

5-5 Monthly variability of global horizontal radiation for Elizabeth City

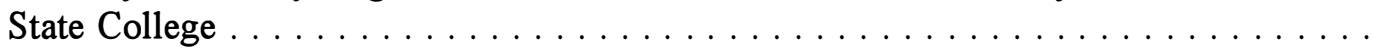

5-6 Monthly variability of direct normal radiation for Elizabeth City

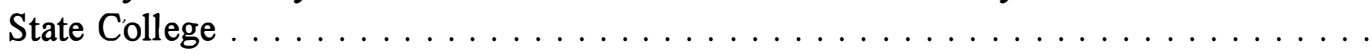

5-7 Monthly variability of global horizontal radiation for Mississippi Valley

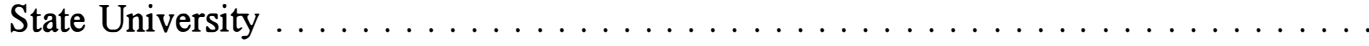

5-8 Monthly variability of direct normal radiation for Mississippi Valley

State University . . . . . . . . . . . . . . . . . . . . .

5-9 Monthly variability of global horizontal radiation for South Carolina

State University . . . . . . . . . . . . . . . . . . . . . . . .

5-10 Monthly variability of direct normal radiation for South Carolina

State University . . . . . . . . . . . . . . . . . . . . . . . . . . . . . . .

5-11 Monthly variability of global horizontal radiation for Savannah State College . . . . 34

5-12 Monthly variability of direct normal radiation for Savannah State College . . . . . . 34 


\section{List of Tables}

$\underline{\text { Table }}$

$\underline{\text { Page }}$

2-1 List of HBCU Stations $\ldots \ldots \ldots \ldots \ldots \ldots \ldots \ldots \ldots \ldots \ldots \ldots$

3-1 Flagging Convention for Global Horizontal, Diffuse Horizontal, and Direct

Normal Solar Radiation . . . . . . . . . . . . . . . . . . . . . . . . . 8

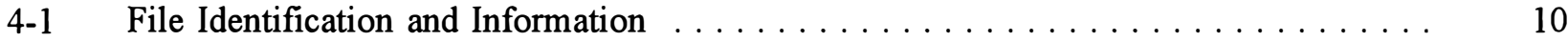

5-1 Daily Minimum and Maximum Global Horizontal Solar Radiation $\left(\mathrm{kWh} / \mathrm{m}^{2}\right) \ldots \ldots$

5-2 Daily Minimum and Maximum Direct Normal Solar Radiation $\left(\mathrm{kWh} / \mathrm{m}^{2}\right) \ldots 14$

5-3 Bethune-Cookman College Global Horizontal Solar Radiation $\left(\mathrm{kWh} / \mathrm{m}^{2}\right) \ldots \ldots$

5-4 Bethune-Cookman College Direct Normal Solar Radiation $\left(\mathrm{kWh} / \mathrm{m}^{2}\right) \ldots \ldots$

5-5 Bluefield State College Global Horizontal Solar Radiation $\left(\mathrm{kWh} / \mathrm{m}^{2}\right) \ldots \ldots \ldots$

5-6 Bluefield State College Direct Normal Solar Radiation $\left(\mathrm{kWh} / \mathrm{m}^{2}\right) \ldots \ldots \ldots$

5-7 Elizabeth City State University Global Horizontal Solar Radiation $\left(\mathrm{kWh} / \mathrm{m}^{2}\right) \ldots \ldots$

5-8 Elizabeth City State University Direct Normal Solar Radiation $\left(\mathrm{kWh} / \mathrm{m}^{2}\right) \ldots \ldots 21$

5-9 Mississippi Valley State University Global Horizontal Solar Radiation $\left(\mathrm{kWh} / \mathrm{m}^{2}\right) \ldots 22$

5-10 Mississippi Valley State University Direct Normal Solar Radiation $\left(\mathrm{kWh} / \mathrm{m}^{2}\right) \ldots 23$

5-11 South Carolina State University Global Horizontal Solar Radiation $\left(\mathrm{kWh} / \mathrm{m}^{2}\right) \ldots \ldots$

5-12 South Carolina State University Direct Normal Solar Radiation $\left(\mathrm{kWh} / \mathrm{m}^{2}\right) \ldots 25$

5-13 Savannah State College Global Horizontal Solar Radiation $\left(\mathrm{kWh} / \mathrm{m}^{2}\right) \ldots \ldots$

5-14 Savannah State College Direct Normal Solar Radiation $\left(\mathrm{kWh} / \mathrm{m}^{2}\right) \ldots \ldots \ldots \ldots$ 


\subsection{Introduction}

Since 1985, the National Renewable Energy Laboratory (NREL), formerly the Solar Energy Research Institute (SERI), has operated a solar radiation measurement network of six stations located at Historically Black Colleges and Universities (HBCUs) in the southeastern United States. NREL initiated this network to provide better regional coverage (Figure 1-1) and to comply with President Reagan's Executive Order 12320, dated September 15, 1981, directing all federal agencies to implement programs to strengthen the nation's HBCUs. Funding for the HBCU network has been provided by the Department of Energy's (DOE's) Resource Assessment Program, Photovoltaic Program, and Solar Thermal Program, and it is currently funded by the Solar Radiation Resource Assessment Project.

The objectives (Hulstrom et al. 1988) of the HBCU network are

- To significantly improve the assessment of solar radiation resources in the southeastern United States

- To enlist the help of the HBCUs in collecting high-quality solar radiation data

- To encourage the distribution of solar radiation resource information and the development of solar energy applications in the Southeast

- To encourage the development of academic and research programs in solar energy at HBCUs.

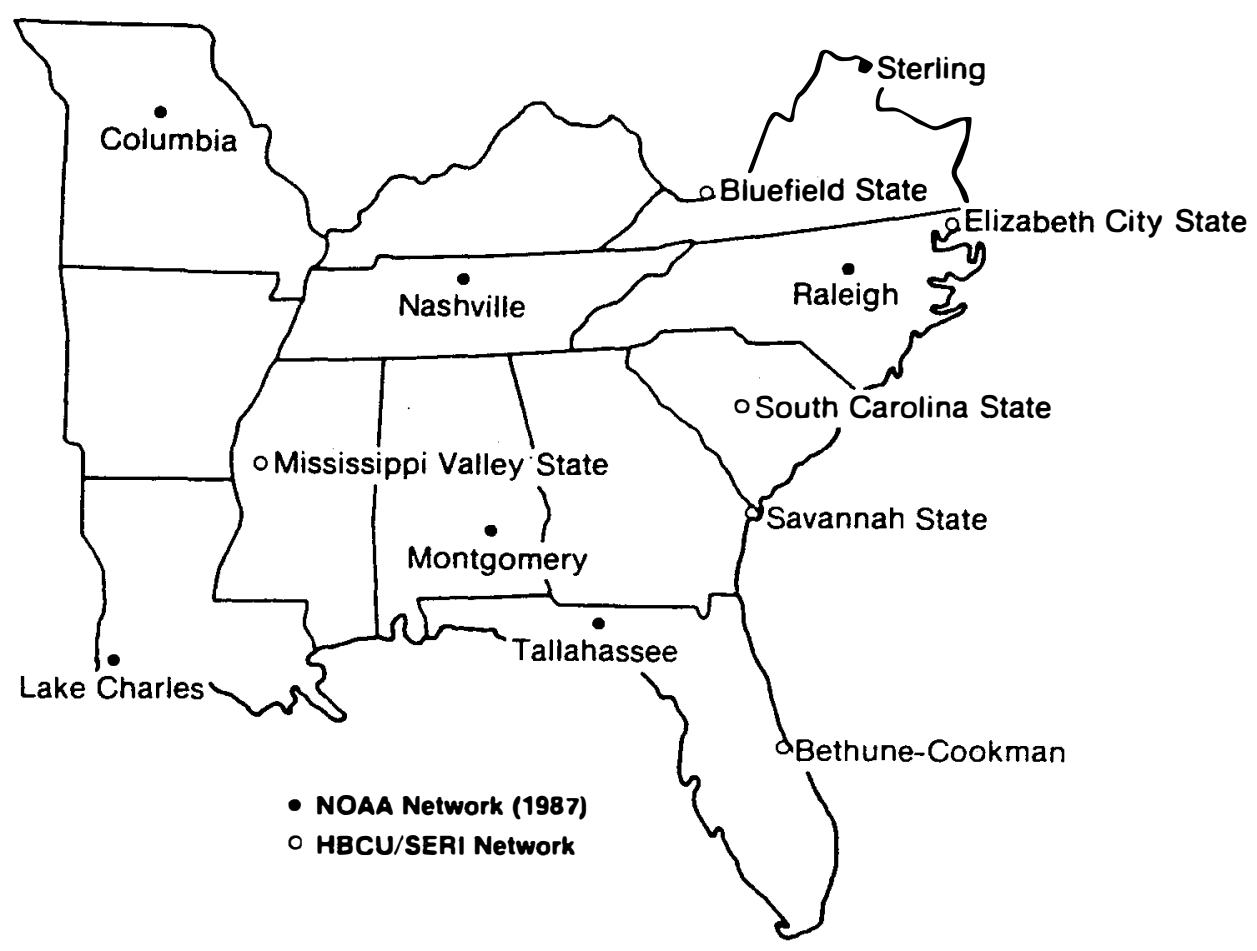

Figure 1-1. The six-station HBCU network provides additional coverage between measurement stations operated by the National Weather Service for the National Oceanic and Atmospheric Administration (NOAA). 
This report presents results for the HBCUs pertaining to the objective of significantly improving solar radiation resource information for the southeastern United States. It provides summary information, such as long-term averages and monthly variability for solar radiation elements, and describes the hourly data sets for the period 1985-1993. The first part of this report describes the HBCU stations, the measurement equipment, and how the data were collected and processed into hourly values. Second, procedures used for quality assessment of the hourly values are presented. Third, the positions of the solar radiation elements in the hourly data sets are defined and sample read statements are given. Fourth, summary information, such as long-term averages and monthly variability, is presented. An appendix is also included to show for each element and station when equipment and calibration factors were changed, and to show the percentage of data that were collected and that passed quality assessment. 


\subsection{Stations and Operations}

This section describes station locations, the solar radiation elements measured, instrument calibration methods, maintenance intervals and procedures, and how the hourly data values were generated.

\subsection{Station Locations}

The HBCU network consists of the six stations shown in Table 2-1. They began collecting data in 1985.

Table 2-1. List of HBCU Stations

\begin{tabular}{|l|l|c|c|c|l|}
\hline Station & Location & $\begin{array}{c}\text { Long. } \\
\left({ }^{\circ} \mathrm{W}\right)\end{array}$ & $\begin{array}{c}\text { Lat. } \\
\left({ }^{\circ} \mathrm{N}\right)\end{array}$ & $\begin{array}{c}\text { Elev. } \\
(\mathrm{m})\end{array}$ & $\begin{array}{c}\text { Time } \\
\text { Zone }\end{array}$ \\
\hline Bethune-Cookman College & Daytona Beach, FL & 81.02 & 29.18 & 20 & Eastern \\
Bluefield State College & Bluefield, WV & 81.24 & 37.26 & 803 & Eastern \\
Elizabeth City State University & Elizabeth City, NC & 76.25 & 36.30 & 4 & Eastern \\
Mississippi Valley State University & Itta Bena, MS & 90.33 & 33.50 & 52 & Central \\
South Carolina State University & Orangeburg, SC & 80.85 & 33.45 & 96 & Eastern \\
Savannah State College & Savannah, GA & 81.07 & 32.03 & 11 & Eastern \\
\hline
\end{tabular}

\subsection{Solar Radiation Measurements}

All stations measure global horizontal radiation and diffuse horizontal radiation. Three stations also have direct normal measurements, but only one of them, Bluefield State College, has made direct normal measurements for the complete period of record. The other two stations with direct normal measurements are Elizabeth City State University, which began making direct normal measurements in March, 1990, and Mississippi Valley State University, which began making direct normal measurements in January, 1993.

Instruments used for measuring solar radiation are an Eppley Laboratory model PSP pyranometer for global horizontal radiation, an Eppley Laboratory model PSP pyranometer with a shadowband for diffuse horizontal radiation, and an Eppley Laboratory model NIP pyrheliometer mounted on a LI-COR model LI-200 sun-following tracker for direct normal radiation. NREL designed a special instrument-mounting platform (see Figure 2-1) for mounting the instruments on building roofs at HBCU stations.

\subsection{Maintenance and Instrument Calibration}

HBCU personnel perform daily -maintenance of the instruments and the data acquisition system. The maintenance includes cleaning the sensors of dirt, moisture, ice, or snow, checking and adjusting the shadowband for proper declination, checking the alignment of the sun trackers and making adjustments (if so equipped), making weather observations, and checking that the sensor outputs are reasonable for the prevailing conditions. A standard log form (Figure 2-2) is used to record maintenance activities. 


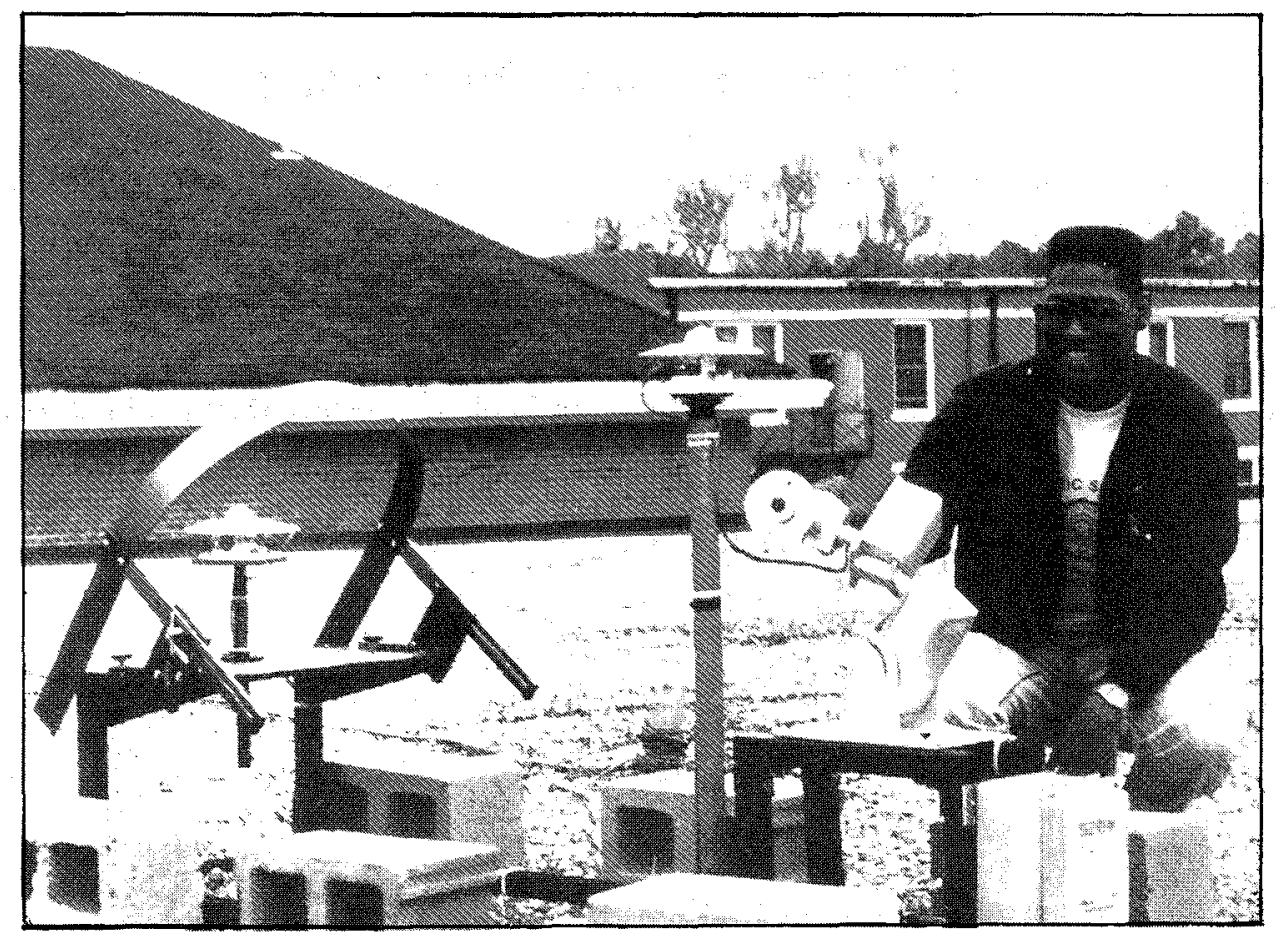

Figure 2-1. Student Eddie Vinson examines the instrument-mounting platform for measuring (left to right) diffuse horizontal, global horizontal, and direct normal radiation at Elizabeth City State University.

Instruments were exchanged with recently calibrated instruments at 1- to 3-year intervals. The Appendix gives the dates for changes of instruments and calibration factors for each solar radiation element and station. Pyranometers and pyrheliometers are calibrated with an absolute cavity radiometer, traceable to the World Radiometric Reference, at NREL's Solar Radiation Research Laboratory. The component summation technique (ASTM 1986) is used to calibrate pyranometers. Pyrheliometers are calibrated by directly comparing their output with that of the absolute cavity radiometer.

\subsection{Data Processing}

The HBCU data acquisition systems scan the solar radiation elements at 10 -second intervals to determine 5-minute averages, which are then saved. Prior to 1993, Campbell Scientific model CR21 datal oggers stored the 5-minute averages on audiocassette tapes, and the tapes were submitted monthly to NREL. In 1993, new Campbell Scientific model CR10 dataloggers with modems were installed. This eliminated the use of the audiocassette tapes, permitting NREL to access the data via telecommunications.

Once at NREL, the data are checked for quality, and diffuse horizontal radiation data are corrected for the presence of the shadowband by methods developed by Drummond for anisotropic skies (Iqbal 1983). Data are then stored on NREL's VAX computer for future use and analysis.

To create the hourly data sets, the 5-minute data values were averaged over the preceding hour. If more than 10 minutes of data in an hour were missing, then the data element was assigned a value of 9900 to indicate missing data. Quality assessment procedures assigned each hourly data value a flag ranging from 0 to 99 , inciicating whether or not the hourly data were reasonable. These procedures are described in the next section. 
Solar Monitoring Station: MAINTENANCE CHECKLIST \& MEATHER LOG

Station Name: Mississippivalleystate For the Period: $11 / 16 / 95$ to 41228

KEY: $\boldsymbol{V}=$ found in $G O O D$ condition $A=$ adjusted

DATE \& TIME

Day of Year

Day of Week

Month/Day/Year

Standard Time

Observer (Initials)

GLOBAL HORIZONTAL

Dome Condition

Sensor Level

Desiccant

DIFFUSE HORIZONTAL

Dome Condition

Sensor Level

Desiccant

Shading Band

DIRECT NORMAL

NIP Window Condition

Tracker Alignment

Signal Cable

DATA ACQUISITION

Time Display

Battery Voltage

Recorder Counter

Tape Change? ( $Y=$ yes)

Printer Status

WEATHER OBSERVATION

Cloud Amount

Temperature

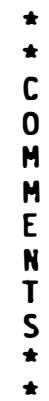

NIP Target:

$\star$
$\star$
$C$
0
$M$
$M$
$E$
$N$
$T$
$S$
$\star$
$X=$ found in $B A D$ condition $0=$ corrected

Clouds: $O=$ Clear $\quad($ Amount $<1 / 10)$

$\Phi=$ Scattered $(1 / 10 \leq$ Amount $<5 / 10)$

(1) $=$ Broken $\quad(5 / 10 \leq$ Amount $<9 / 10)$

$\bigoplus=0$ vercast (Amount $\geq 9 / 10$ )

ơ

\begin{tabular}{l|l|l|l|l|l|l|}
\hline $5 A$ & 50 & Mi & TU & WE & +N & FR \\
\hline 16 & 17 & 18 & 19 & 20 & 21 & 22 \\
\hline $6: 50$ & $7: 20$ & $7: 01$ & $6: 40$ & $7: 35$ & $6: 57$ & $6: 30$ \\
\hline $5 E$ & $5 E$ & $S E$ & 51 & $5 F$ & 51 & $5 F$ \\
\hline
\end{tabular}
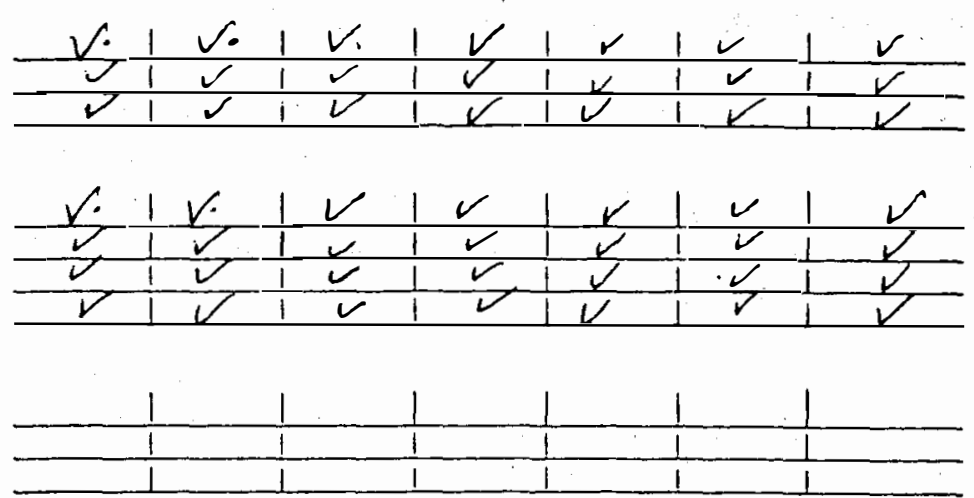

产点

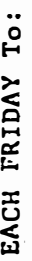

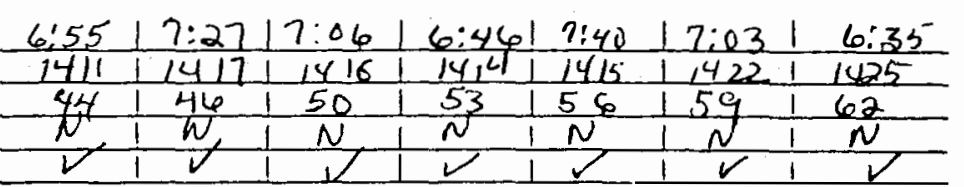

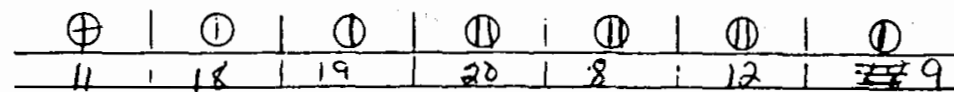

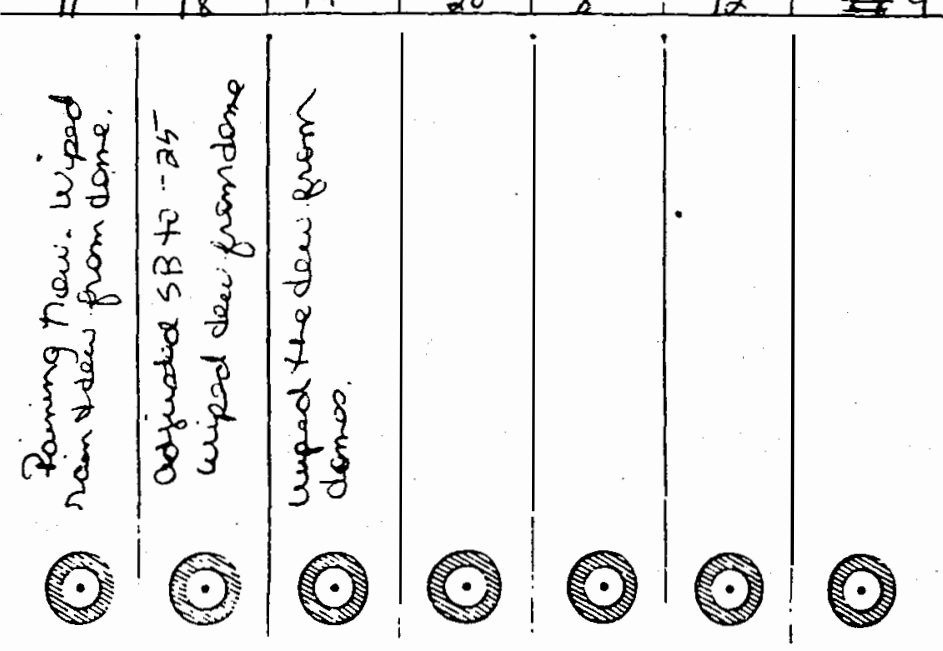

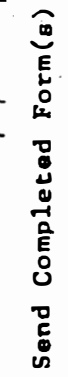

Figure 2-2. Log used to record maintenance of instruments and data acquisition system 


\subsection{Quality Assessment of Hourly Data}

After data are collected, quality assessment can be performed to indicate whether a data value is reasonable, too small, too large, or missing. It is not used to change data values, and should not be confused with quality control and quality assurance. Quality control and quality assurance occur before and during data collection and include procedures such as the proper selection and installation of instruments and data acquisition equipment, as well as regular maintenance and calibration. Quality assessment cannot replace quality control and quality assurance because it will not detect small changes caused by dirty or unleveled sensors or changes in calibration factors.

Quality assessment procedures for the solar radiation data assign a flag ranging from 0 to 99 to each of the hourly data elements. To select data for analysis purposes, the flags may be used to screen the data files for data meeting user-defined acceptance criteria.

\subsection{Quality Assessment for Solar Radiation Data}

The three solar radiation data elements—global horizontal, diffuse horizontal, and direct normal—are quality assessed using SERI QC, a procedural and software package developed by NREL (1993). SERI QC defines ranges of acceptable data, depending on whether one, two, or all three hourly data elements are present. Ranges are defined based on dimensionless parameters normalized with respect to extraterrestrial radiation, where

$$
\begin{aligned}
\mathrm{K}_{\mathrm{t}} & =\text { Clearness index or global horizontal transmittance } \\
& =\text { Global horizontal radiation } \div \text { extraterrestrial horizontal radiation } \\
\mathrm{K}_{\mathrm{d}} & =\text { Diffuse horizontal transmittance } \\
& =\text { Diffuse horizontal radiation } \div \text { extraterrestrial horizontal radiation } \\
\mathrm{K}_{\mathrm{n}} & =\text { Direct normal transmittance } \\
& =\text { Direct normal radiation } \div \text { extraterrestrial direct normal radiation. }
\end{aligned}
$$

Depending on the circumstances, SERI QC performs one-element, two-element, or three-element tests. First, it performs a one-element test by defining a range of acceptable values between minimum and maximum values of $\mathrm{K}_{\mathrm{t}}, \mathrm{K}_{\mathrm{d}}$, or $\mathrm{K}_{\mathrm{n}}$, depending on the element being tested, based on three air mass regimes and the month of the year.

Second, if the zenith angle (at the middle of the hour) is less than or equal to $80^{\circ}$, and all three of the elements are present, SERI QC performs a three-element test by defining a range of acceptable values so that the equation $\mathrm{K}_{t}=\mathrm{K}_{\mathrm{d}}+\mathrm{K}_{\mathrm{n}}$ is satisfied within an arbitrary error limit of \pm 0.03 , which accounts for measurement uncertainties.

Third, if the data pass the three-element test (or only two elements passed the one-element test), SERI QC performs a two-element test by defining a range of acceptable values within boundaries such as those shown in Figure 3-1. The boundaries in the figure are previously determined empirically for three different air mass regimes for each month using data collected at the site. In Figure 3-1, the direct normal transmittance is plotted against global horizontal transmittance. The lower boundary illustrates that with increasing cloud cover, direct normal radiation decreases more rapidly than global horizontal radiation. Consequently, data for cloudy skies are grouped toward the lower boundary. ' Clear sky data reside near the upper boundary. 


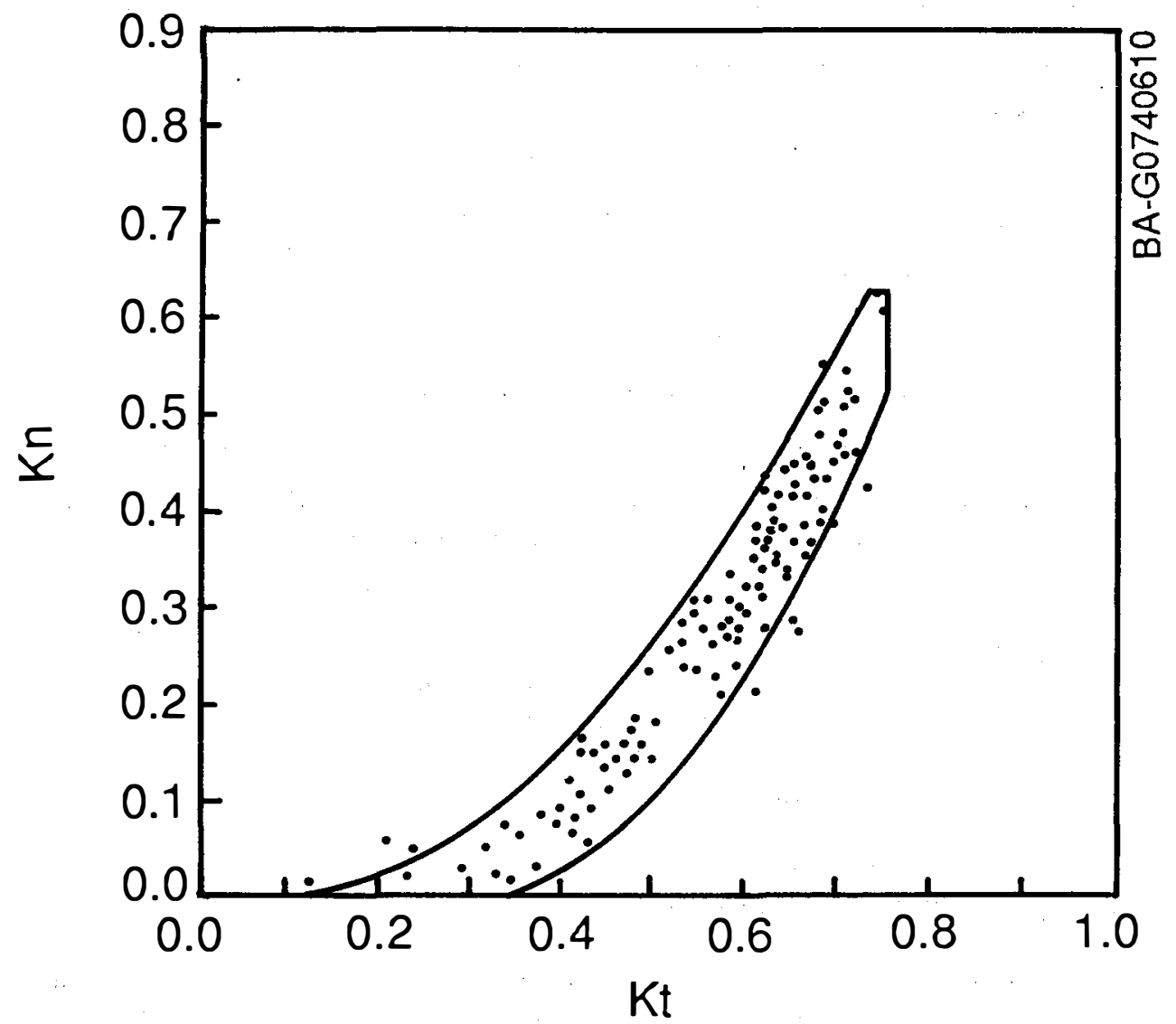

Figure 3-1. SERI QC data boundaries for two-element quality assessment

For some seldom occurring conditions, data depicting real conditions may reside outside the boundaries and be flagged as bad data. For example, if the sun is near the edge of a cloud, some of the sun's rays can be reflected off the edge of the cloud and increase the global horizontal radiation and $\mathrm{K}_{\mathrm{t}}$ without affecting the direct normal radiation and $K_{n}$. This shifts the data point to the right in Figure 3-1, and it may be to the right of the lower boundary if $\mathrm{K}_{\mathrm{t}}$ is large enough.

Bad data can also be flagged good by SERI QC, as was seen for some diffuse horizontal data for Bethune-Cookman College (see Section 5). For overcast sky conditions, SERI QC does not detect an improperly adjusted shadowband because $\mathrm{K}_{\mathrm{n}}=0$ and $\mathrm{K}_{\mathrm{t}}=\mathrm{K}_{\mathrm{d}}$ within its arbitrary error limit.

After all SERI QC tests are completed, flags are assigned to the data according to the convention listed in Table 3-1. 
Table 3-1. Flagging Convention for Global Horizontal, Diffuse Horizontal, and Direct Normal Solar Radiation

\begin{tabular}{|c|c|c|}
\hline Flag & \multicolumn{2}{|l|}{ Description } \\
\hline 0 & \multicolumn{2}{|c|}{ Untested data } \\
\hline 1 & \multicolumn{2}{|c|}{ Passed one-element test; data within minimum-maximum limits of $\mathrm{K}_{\mathrm{t}}, \mathrm{K}_{\mathrm{d}}$, or $\mathrm{K}_{\mathrm{n}}$} \\
\hline 2 & \multicolumn{2}{|c|}{ Passed two-element test; data within \pm 0.03 of boundaries } \\
\hline 3 & \multicolumn{2}{|c|}{ Passed three-element test; data within \pm 0.03 of satisfying $K_{t}=K_{d}+K_{n}$} \\
\hline 7 & \multicolumn{2}{|c|}{ Failed one-element test; data below allowed minimum } \\
\hline 8 & \multicolumn{2}{|c|}{ Failed one-element test; data above allowed maximum } \\
\hline 9 & \multicolumn{2}{|c|}{ Passed three-element test but failed two-element test by greater than 0.05} \\
\hline \multirow[t]{7}{*}{$10-93$} & \multicolumn{2}{|c|}{$\begin{array}{l}\text { Failed two- or three-element test in one of four ways: To determine the test failed } \\
\text { and the manner of the failure (high or low), examine the remainder of the } \\
\text { calculation }(\text { flag }+2) / 4 \text {. }\end{array}$} \\
\hline & Remainder & Failure \\
\hline & & Element too low by three-element test \\
\hline & & Element too high by three-element test \\
\hline & & Element too low by two-element test \\
\hline & & Element too high by two-element test \\
\hline & \multicolumn{2}{|c|}{$\begin{array}{l}\text { The magnitude of the test failure (distance in K-units) is determined by the } \\
\text { calculation: } d=[\operatorname{INT}(\text { flag }+2) / 4] / 100\end{array}$} \\
\hline $94-97$ & \multicolumn{2}{|c|}{$\begin{array}{l}\text { Data fall into a physically impossible region where } K_{n}>K_{t} \text { by } K \text {-space distances } \\
\text { of } 0.05 \text { to } 0.10 \text { (94), } 0.10 \text { to } 0.15 \text { (95), } 0.15 \text { to } 0.20(96) \text {, and } \geq 0.20 \text { (97) }\end{array}$} \\
\hline 99 & \multicolumn{2}{|c|}{ Missing data } \\
\hline
\end{tabular}




\subsection{Hourly Data Files}

The HBCU hourly data set consists of six data files, one for each station. This section describes the data format, identifies the location of each element in a line of data, provides sample read statements for three computer languages, and informs how the hourly data sets may be obtained.

\subsection{File Format}

Each line of the data file contains the time and the data elements with their respective quality assessment (QA) flags. There is one line of data for each hour of the day. Hour values are from 1 to 24 (local standard time) and they correspond to data collected for the preceding hour. For example, an hour value of 16 is used for data averaged over the hour from 3 p.m. to 4 p.m.

Within a file, data are presented for each hour and day from the beginning to the end of the file. If an element value was missing, a data value of 9900 and a QA flag value of 99 were assigned. For sites where direct normal radiation was not measured, the missing data value of 9900 and a QA flag value of 99 were assigned to preserve a common format for all HBCU stations.

Figure 4-1 shows a sample printout of a portion of a data file. The first line in Figure 4-1 is for illustrative purposes and is not a part of the data file. The time and data elements for each line of data are presented in the following order: year (YR), month (MO), day (DY), hour (HR), global horizontal radiation (GH), diffuse horizontal radiation (DIF), and direct normal radiation (DN). Quality assessment flags (FL) follow each of the data elements. The data values and flags are expressed as whole numbers and the units for the solar radiation elements are $\mathrm{Wh} / \mathrm{m}^{2}$.

$\begin{array}{rrrrrrrrrr}\text { YR } & \text { MO } & \text { DY } & \text { HR } & \text { GH } & \text { FL } & \text { DIF } & \text { FL } & \text { DN } & \text { FL } \\ 85 & 7 & 4 & 1 & -1 & 1 & -1 & 1 & 9900 & 99 \\ 85 & 7 & 4 & 2 & -1 & 1 & -1 & 1 & 9900 & 99 \\ 85 & 7 & 4 & 3 & -1 & 1 & -2 & 1 & 9900 & 99 \\ 85 & 7 & 4 & 4 & -2 & 1 & -2 & 1 & 9900 & 99 \\ 85 & 7 & 4 & 5 & 0 & 1 & -1 & 1 & 9900 & 99 \\ 85 & 7 & 4 & 6 & 53 & 1 & 43 & 1 & 9900 & 99 \\ 85 & 7 & 4 & 7 & 97 & 2 & 98 & 2 & 9900 & 99 \\ 85 & 7 & 4 & 8 & 350 & 2 & 271 & 2 & 9900 & 99 \\ 85 & 7 & 4 & 9 & 435 & 2 & 302 & 2 & 9900 & 99 \\ 85 & 7 & 4 & 10 & 537 & 2 & 405 & 2 & 9900 & 99 \\ 85 & 7 & 4 & 11 & 730 & 2 & 375 & 2 & 9900 & 99 \\ 85 & 7 & 4 & 12 & 942 & 2 & 244 & 2 & 9900 & 99 \\ 85 & 7 & 4 & 13 & 924 & 2 & 257 & 2 & 9900 & 99 \\ 85 & 7 & 4 & 14 & 920 & 2 & 185 & 2 & 9900 & 99 \\ 85 & 7 & 4 & 15 & 657 & 2 & 224 & 2 & 9900 & 99 \\ 85 & 7 & 4 & 16 & 430 & 2 & 274 & 2 & 9900 & 99 \\ 85 & 7 & 4 & 17 & 371 & 2 & 187 & 2 & 9900 & 99 \\ 85 & 7 & 4 & 18 & 232 & 2 & 146 & 2 & 9900 & 99 \\ 85 & 7 & 4 & 19 & 50 & 1 & 43 & 1 & 9900 & 99 \\ 85 & 7 & 4 & 20 & 3 & 1 & 2 & 1 & 9900 & 99 \\ 85 & 7 & 4 & 21 & -2 & 1 & -3 & 1 & 9900 & 99 \\ 85 & 7 & 4 & 22 & -2 & 1 & -2 & 1 & 9900 & 99 \\ 85 & 7 & 4 & 23 & -2 & 1 & -2 & 1 & 9900 & 99 \\ 85 & 7 & 4 & 24 & -2 & 1 & -2 & 1 & 9900 & 99\end{array}$

Figure 4-1. Data for Mississippi Valley State University for July 4, 1985 


\subsection{File Identification}

Table 4-1 presents file names assigned to each station's data file, the period of time associated with the data file, and the date of the first recorded measurement for each of the solar radiation elements. The data files contain complete months of data; consequently, the first month of each file may contain missing data records until the day when measurements began.

Table 4-1. File Identification and Information

\begin{tabular}{|l|r|r|r|r|c|}
\hline Station & File Name & Period & \multicolumn{3}{|c|}{ First Recorded Measuremen } \\
\cline { 4 - 6 } & & & Global & Diffuse & Direct \\
\hline Bethune-Cookman College & BC.DAT & $8 / 85-12 / 93$ & $8 / 2 / 85$ & $8 / 2 / 85$ & None \\
Bluefield State College & BS.DAT & $11 / 85-12 / 93$ & $11 / 6 / 85$ & $11 / 6 / 85$ & $11 / 6 / 85$ \\
Elizabeth City State University & EC.DAT & $9 / 85-12 / 93$ & $9 / 3 / 85$ & $9 / 3 / 85$ & $3 / 1 / 90$ \\
Mississippi Valley State University & MV.DAT & $7 / 85-12 / 93$ & $7 / 1 / 85$ & $7 / 1 / 85$ & $1 / 1 / 93$ \\
South Carolina State College & SC.DAT & $8 / 85-12 / 93$ & $8 / 1 / 85$ & $8 / 1 / 85$ & None \\
Savannah State College & SS.DAT & $8 / 85-12 / 93$ & $8 / 29 / 85$ & $8 / 29 / 85$ & None \\
\hline
\end{tabular}

\subsection{Sample Read Statements}

The HBCU data files contain ASCII characters that are readable using various computer languages. Sample read statements for three computer languages are shown below.

\section{IBM BASIC}

100 INPUT \#1, YEAR, MON, DAY, HOUR

110 FOR I = 1 TO 3: INPUT \#1, X(I), Y(I): NEXT I

\section{FORTRAN}

$\begin{array}{ll}\text { READ }(16,100) \text { YEAR, MON, } \\ 100 & \text { FORMAT( 4I3, } 3(\text { F5.0, I3 ) ) }\end{array}$

C

fscanf ( fp_in, "\%d \%d \%d \%d", \&YEAR, \&MON, \&DAY, \&HOUR ); for ( $I=1 ; \mathrm{I}<=3$; I++ ) fscanf ( fp_in, "\%f \%d", \&X[I], \&Y[I] );

where:

$$
\begin{aligned}
\text { YEAR = } & \text { last two digits of year }(89=1989) \\
\text { MON }= & \text { month of year, } 1 \text { to } 12 \\
\text { DAY }= & \text { day of month, } 1 \text { to } 31 \\
\text { HOUR = } & \text { hour of day, } 1 \text { to } 24 \\
\mathrm{X}(\mathrm{I})= & \text { data value for Ith element, read as floating point number in FORTRAN and C } \\
\mathrm{Y}(\mathrm{I})= & \text { QA flag for Ith element, read as integer number in FORTRAN and C } \\
& (\mathrm{I}=1 \text { for global, } \mathrm{I}=2 \text { for diffuse, and I = } 3 \text { for direct }) .
\end{aligned}
$$




\subsection{Obtaining the HBCU Data Sets}

The HBCU hourly data sets may be obtained by contacting the NREL Technical Inquiry Service at 303/275-4099. The data sets are provided on three MS-DOS ${ }^{\circledR}$ formatted 1.44-MB floppy disks. The data files on the floppy disks are compressed to minimize their file size. Uncompressed, the six data files require a total of about $16.7 \mathrm{MB}$ of disk space. The necessary program to uncompress the data files is included on the floppy disks. A "readme" file describes the procedure for uncompressing the data files and installing them on a computer's hard disk. 


\subsection{Summary Data}

For each HBCU station, summary information pertaining to the amount of data missing and the amount passing quality criteria was determined. Using the quality checked data and procedures to address any missing data, monthly and annual solar radiation values for global horizontal and direct normal radiation were calculated and presented in tables and graphs.

\subsection{Missing Data and Quality Assessed Data Summaries}

Appendix A contains tables providing quality assessment summaries for daylight hours for each month of the period of record for each HBCU station. For measured values of global horizontal radiation, diffuse horizontal radiation, and direct normal radiation, the tables give two numbers for each month: (1) the percentage of possible data collected and (2) the percentage of data that passes their quality assessment. The percentages are based on the total daylight hours possible for each month.

The pass criteria for measured data were that it was within 0.05 of the boundaries during the SERI QC two-element test, or within \pm 0.05 of satisfying the equation $\mathrm{K}_{t}=\mathrm{K}_{\mathrm{d}}+\mathrm{K}_{\mathrm{n}}$ for the three-element test. These criteria correspond to SERI QC flags 1-3 and 10-21.

For stations that did not make direct normal measurements, or if the direct normal data are missing or failed their quality assessment, direct normal radiation may be calculated using global horizontal radiation and diffuse horizontal radiation measurements (if these two measurements pass their quality assessment). Therefore, besides the quality assessment table for measured direct normal data, the appendix includes two additional tables concerning direct normal radiation calculated from global horizontal and diffuse horizontal radiation measurements.

The first table indicates the percentage of direct normal data that is available either from direct normal measurements that pass the SERI QC criteria, or from calculating direct normal radiation by using measured values of global horizontal and diffuse horizontal radiation that pass SERI QC criteria.

The second table imposes an additional check to make sure that the shadowband was being routinely adjusted. In brief, the check examined diffuse horizontal SERI QC flags when global horizontal radiation was at or above thresholds that indicated direct normal radiation was present. If the diffuse horizontal SERI QC flag was a passing value, then the shadow band was assumed properly adjusted and the calculated direct normal value was given a passing mark. If the diffuse horizontal SERI QC flag was not a passing value, then the shadowband was assumed to be not properly adjusted and the calculated direct normal values were assumed invalid until such time that the test was passed, indicating that the shadowband was brought into adjustment.

For stations other than Bethune-Cookman College, both the first and second tables for calculated direct normal radiation have essentially the same numbers. For Bethune-Cookman College, the numbers are much different for 1991-1993. Using SERI QC alone to check the data permitted a large amount of data collected under cloudy skies and conditions of low direct beam radiation to be assigned passing flags, because the radiation detected by the shadowband pyranometer is not as sensitive to the alignment of the shadowband under these conditions. The additional shadowband check permitted better screening of the data. 


\subsection{Influence of Missing Data on Summary Data Uncertainties}

When calculating the average daily radiation for a period, both missing data and the measurement uncertainty of the instruments that measured the radiation create an uncertainty in the calculated value.

The total measurement uncertainties of pyranometers and pyrheliometers have previously been established by Stoffel et al. (1987) by using the method of Abernethy and Ringhiser (1985). This rootsum-square method defines an uncertainty interval $\pm U_{R s S}$ having a $95 \%$ confidence level.

$$
\mathrm{U}_{\mathrm{RSS}}=\left[(\mathrm{tR})^{2}+\mathrm{B}^{2}\right]^{1 / 2}
$$

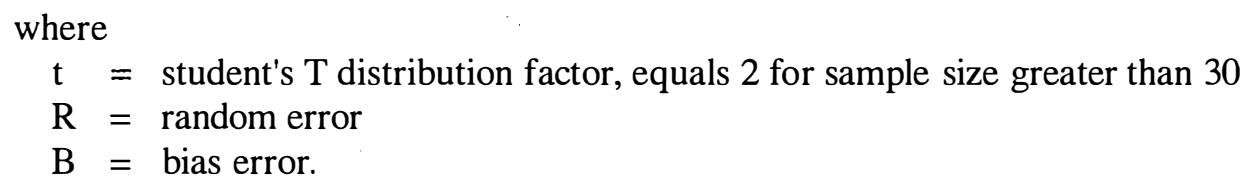

For pyranometers, the total uncertainty was determined to be $4.4 \%$ (3.1\% bias and $1.6 \%$ random), and for pyrheliometers the total uncertainty was determined to be $3.2 \%$ ( $1.8 \%$ bias and $1.3 \%$ random). These uncertainties apply to single measurements and include errors caused by temperature response, cosine and azimuth response, linearity, spectral response, installation, sensor cleanliness, and data acquisition equipment. For this work, when summing measurements to calculate the average daily radiation for a month, the large number of measurements (sample rate of once every 10 seconds) cause the random errors to cancel each other. Consequently, only the bias error remains (3.1\% for pyranometers and $1.8 \%$ for pyrheliometers).

Besides the instrument bias error, additional uncertainties in the calculation of the average daily radiation for a month exist if data are missing because of instrument or data acquisition system malfunction. For this analysis, data not meeting their quality assessment criteria are also considered missing. If data for a day are missing, we do not know what the data value should be, but we can expect it to be between a minimum and maximum value. For the HBCU network, these minimum and maximum values are shown for global horizontal and direct normal radiation in Tables 5-1 and 5-2, respectively. They were determined by calculating the solar radiation for each day during the period of record when data were available.

The effect of missing data on the uncertainty of the calculated average daily radiation for a month is addressed by: (1) assuming the radiation for a missing day is the same as the average daily radiation calculated using the available data for the month, (2) assigning a random error to the assumed radiation value that is equal to the absolute difference between the assumed radiation value and the minimum or maximum value, whichever is larger, from Tables 5-1 or 5-2, and (3) combining the random error resulting from missing data with the bias error for the instrument to determine the total uncertainty.

The random error resulting from missing data during a month can be evaluated by the method outlined by Holman (1971), where $F$ is a given function of independent variables $X_{1}, X_{2}, \ldots X_{N}$.

$$
F=F\left(X_{1}, X_{2}, \ldots X_{N}\right)
$$

Letting $R$ be the uncertainty in the result and $R_{1}, R_{2}, \ldots R_{N}$ be the uncertainty in the independent variables:

$$
\mathrm{R}=\left[\left(\mathrm{R}_{1} \partial \mathrm{F} / \partial \mathrm{X}_{1}\right)^{2}+\left(\mathrm{R}_{2} \partial \mathrm{F} / \partial \mathrm{X}_{2}\right)^{2}+\ldots\left(\mathrm{R}_{\mathrm{N}} \partial \mathrm{F} / \partial \mathrm{X}_{\mathrm{N}}\right)^{2}\right]^{1 / 2}
$$


Table 5-1. Daily Minimum and Maximum Global Horizontal Solar Radiation $\left(\mathrm{kWh} / \mathrm{m}^{2}\right)$

\begin{tabular}{|c|c|c|c|c|c|c|c|c|c|c|c|c|}
\hline \multirow[b]{2}{*}{ Month } & \multicolumn{2}{|c|}{$\begin{array}{c}\text { Bethune- } \\
\text { Cookman } \\
\text { College }\end{array}$} & \multicolumn{2}{|c|}{$\begin{array}{c}\text { Bluefield } \\
\text { State College }\end{array}$} & \multicolumn{2}{|c|}{$\begin{array}{l}\text { Elizabeth } \\
\text { City State } \\
\text { University } \\
\end{array}$} & \multicolumn{2}{|c|}{$\begin{array}{l}\text { Mississippi } \\
\text { Valley State } \\
\text { University }\end{array}$} & \multicolumn{2}{|c|}{$\begin{array}{c}\text { South } \\
\text { Carolina State } \\
\text { University }\end{array}$} & \multicolumn{2}{|c|}{$\begin{array}{c}\text { Savannah } \\
\text { State College }\end{array}$} \\
\hline & Min & $\operatorname{Max}$ & Min & Max & Min & Max & Min & Max & Min & Max & Min & Max \\
\hline Jan & 1.2 & 4.6 & 0.3 & 3.8 & 0.4 & 3.8 & 0.4 & 4.2 & 0.6 & 4.4 & 0.5 & 4.4 \\
\hline Feb & 1.4 & 5.9 & 0.5 & 5.2 & 0.4 & 5.4 & 0.7 & 5.4 & 0.9 & 5.4 & 1.0 & 5.7 \\
\hline Mar & 1.4 & 7.1 & 0.5 & 6.8 & 0.9 & 6.7 & 1.1 & 7.0 & 1.3 & 7.1 & 1.6 & 7.0 \\
\hline Apr & 3.2 & 7.8 & 0.7 & 8.1 & 1.2 & 7.9 & 0.9 & 8.0 & 1.8 & 8.2 & 2.2 & 8.1 \\
\hline May & 3.9 & 8.4 & 1.1 & 8.5 & 1.2 & 8.7 & 2.0 & 8.5 & 1.2 & 8.7 & 2.2 & 8.2 \\
\hline June & 2.3 & 8.3 & 1.8 & 8.6 & 2.3 & 8.5 & 2.3 & 8.7 & 1.2 & 8.6 & 1.6 & 8.4 \\
\hline July & 2.3 & 8.1 & 1.8 & 8.9 & 2.9 & 8.4 & 3.2 & 8.1 & 1.5 & 8.4 & 2.4 & 8.2 \\
\hline Aug & 2.0 & 7.6 & 1.1 & 7.5 & 1.1 & 7.6 & 2.7 & 7.7 & 0.7 & 7.8 & 2.4 & 7.2 \\
\hline Sept & 1.2 & 6.9 & 1.0 & 6.9 & 1.1 & 6.7 & 1.4 & 7.0 & 0.8 & 6.7 & 1.0 & 6.7 \\
\hline Oct & 1.8 & 6.2 & 0.7 & 5.3 & 0.7 & 5.6 & 0.7 & 5.9 & 0.8 & 6.0 & 1.2 & 6.1 \\
\hline Nov & 1.1 & 4.9 & 0.4 & 4.0 & 0.6 & 4.1 & 0.5 & 4.4 & 0.9 & 4.5 & 0.6 & 4.7 \\
\hline Dec & 0.9 & 4.0 & 0.3 & 3.2 & 0.3 & 3.3 & 0.6 & 3.7 & 0.6 & 3.6 & 0.7 & 3.7 \\
\hline
\end{tabular}

Table 5-2. Daily Minimum and Maximum Direct Normal Solar Radiation (kWh/m²)

\begin{tabular}{|c|c|c|c|c|c|c|c|c|c|c|c|c|}
\hline \multirow[b]{2}{*}{ Month } & \multicolumn{2}{|c|}{$\begin{array}{c}\text { Bethune- } \\
\text { Cookman } \\
\text { College } \\
\end{array}$} & \multicolumn{2}{|c|}{$\begin{array}{c}\text { Bluefield } \\
\text { State College }\end{array}$} & \multicolumn{2}{|c|}{$\begin{array}{l}\text { Elizabeth } \\
\text { City State } \\
\text { University } \\
\end{array}$} & \multicolumn{2}{|c|}{$\begin{array}{l}\text { Mississippi } \\
\text { Valley State } \\
\text { University }\end{array}$} & \multicolumn{2}{|c|}{$\begin{array}{c}\text { South } \\
\text { Carolina State } \\
\text { University }\end{array}$} & \multicolumn{2}{|c|}{$\begin{array}{c}\text { Savannah } \\
\text { State College }\end{array}$} \\
\hline & Min & Max & Min & Max & Min & Max & Min & $\operatorname{Max}$ & Min & $\operatorname{Max}$ & Min & Max \\
\hline Jan & 0.0 & 8.4 & 0.0 & 8.1 & 0.0 & 8.6 & 0.0 & 8.4 & 0.0 & 8.5 & 0.0 & 8.6 \\
\hline Feb & 0.0 & 9.6 & 0.0 & 9.4 & 0.0 & 9.6 & 0.0 & 9.3 & 0.0 & 9.2 & 0.0 & 9.3 \\
\hline Mar & 0.0 & 9.5 & 0.0 & 9.7 & 0.0 & 9.4 & 0.0 & 10.1 & 0.0 & 10.2 & 0.2 & 10.0 \\
\hline Apr & 0.8 & 9.9 & 0.0 & 10.4 & 0.0 & 9.7 & 0.0 & 10.6 & 0.0 & 10.2 & 0.0 & 10.1 \\
\hline May & 0.2 & 10.5 & 0.0 & 10.9 & 0.0 & 10.5 & 0.0 & 10.8 & 0.0 & 10.4 & 0.4 & 9.9 \\
\hline June & 0.2 & 9.7 & 0.0 & 10.6 & 0.0 & 9.4 & 0.0 & 11.2 & 0.0 & 10.1 & 0.0 & 8.7 \\
\hline July & 0.0 & 9.3 & 0.0 & 11.7 & 0.1 & 9.0 & 0.3 & 9.5 & 0.3 & 9.4 & 0.8 & 9.9 \\
\hline Aug & 0.6 & 8.9 & 0.0 & 9.5 & 0.0 & 8.9 & 0.0 & 9.3 & 0.0 & 9.0 & 0.0 & 8.0 \\
\hline Sept & 0.0 & 8.2 & 0.0 & 10.1 & 0.0 & 8.5 & 0.0 & 9.8 & 0.0 & 9.1 & 0.0 & 8.7 \\
\hline Oct & 0.0 & 8.9 & 0.0 & 9.1 & 0.0 & 8.8 & 0.0 & 9.3 & 0.0 & 9.5 & 0.0 & 9.5 \\
\hline Nov & 0.0 & 8.5 & 0.0 & 8.2 & 0.0 & 8.2 & 0.0 & 8.3 & 0.0 & 8.4 & 0.0 & 8.2 \\
\hline Dec & 0.0 & 8.2 & 0.0 & 7.8 & 0.0 & 7.7 & 0.0 & 8.0 & 0.0 & 7.9 & 0.0 & 7.9 \\
\hline
\end{tabular}


If the function $\mathrm{F}$ is defined as the average of $\mathrm{n}$ values, then the uncertainty in the average becomes:

$$
R=(1 / n)\left[\left(R_{1}\right)^{2}+\left(R_{2}\right)^{2}+\ldots\left(R_{N}\right)^{2}\right]^{1 / 2}
$$

As applied to our need to determine the random error created by missing data, Eq. 4 can be expressed as:

where:

$$
\mathrm{R}_{\mathrm{m}}=100\left(\mathrm{R}_{\mathrm{i}} / \mathrm{I}\right)[(1-\mathrm{x}) / \mathrm{m}]^{1 / 2}
$$

$$
\begin{aligned}
\mathrm{R}_{\mathrm{m}}= & \text { percentage of random error in calculated average daily radiation for the month } \\
\mathrm{I}= & \text { calculated average daily radiation for a month using data passing quality assessment } \\
\mathrm{R}_{\mathrm{i}}= & \text { random error associated with substituting the average daily radiation for the month } \\
& \text { for missing data. Equals the larger of ( } \mathrm{I}-\text { Min ) or (Max - I ). Min and Max from } \\
& \text { Table } 5-1 \text { or Table 5-2. } \\
\mathrm{x}= & \text { fraction of data during the month that passes quality assessment (from Appendix) } \\
\mathrm{m}= & \text { number of days during the month. }
\end{aligned}
$$

Expressing the total uncertainty $\mathrm{U}$ of the calculated average daily radiation for the month as a function of both the random error $\mathrm{R}_{\mathrm{m}}$ resulting from missing data and the instrument bias error $\mathrm{B}$ :

$$
\mathrm{U}=\left(\mathrm{R}_{\mathrm{m}}{ }^{2}+\mathrm{B}^{2}\right)^{1 / 2}
$$

This method appears to work well ( $95 \%$ confidence level) as long as no more than $30 \%$ of the data during the month are missing or fail their quality assessment (Marion 1993). When larger quantities of data are missing, the relationship is not favorable. Although we treat the missing data as a random error, solar radiation itself is not random and is influenced by season, geographical features, and climate. Consequently, when large segments of data are missing, the error may not always be random.

Equation 5 can also be used to stress the importance of maximum data recovery when measuring solar radiation to calculate monthly averages for resource assessment. With only 1 day of global horizontal radiation data missing, the error introduced in the monthly average because of missing data can be as large as the bias error of the instrument. For direct normal radiation, the error in the monthly average because of 1 missing day of data can be twice as large as the bias error of the instrument. Direct normal radiation is more variable, and the pyrheliometer measuring it has less instrument error than the pyranometer measuring global solar radiation.

\subsection{Average Daily Radiation}

The average daily radiation by month and year are shown in Tables 5-3 through 5-14 for global horizontal radiation and direct normal radiation. The percentage of uncertainty for each average was determined using Eqs. 5 and 6 and is shown in parenthesis below the average. For months with more than $30 \%$ of the data missing or failing their quality assessment, no average was calculated because insufficient data were present to adequately estimate an uncertainty. When direct normal radiation was calculated using global horizontal and diffuse horizontal radiation, the check for proper alignment of the shadowband was included as part of the quality assessment.

In the right column of the tables, annual averages were determined if all the monthly averages were present. The bottom row shows averages by month for the period of record. These were determined using values displayed in the rows above. The annual average in the bottom row was determined using the monthly averages in the bottom row. 
Table 5-3. Bethune-Cookman College Global Horizontal Solar Radiation $\left(\mathrm{kWh} / \mathrm{m}^{2}\right)$

(Percentage Uncertainty Shown in Parenthesis, Asterisks Denote Insufficient Data)

\begin{tabular}{|c|cccccccccccc|c|}
\hline Year & Jan & Feb & Mar & Apr & May & June & July & Aug & Sept & Oct & Nov & Dec & Ann \\
\hline \multirow{2}{*}{85} & & & & & & & & 4.74 & 4.91 & 4.28 & 3.46 & 3.04 & \\
& & & & & & & & $(5.9)$ & $(5.9)$ & $(3.8)$ & $(4.7)$ & $(3.6)$ & \\
86 & 3.31 & 3.86 & 5.22 & 6.87 & $* * * *$ & 5.59 & $* * * *$ & $* * * *$ & 4.92 & $* * * *$ & $* * * *$ & $* * * *$ & \\
& $(4.9)$ & $(6.4)$ & $(5.5)$ & $(3.7)$ & & $(5.9)$ & & & $(7.2)$ & & & \\
87 & $* * * *$ & $* * * *$ & $* * * *$ & $* * * *$ & $* * * *$ & $* * * *$ & 6.47 & $* * * *$ & $* * * *$ & 4.33 & $* * * *$ & $* * * *$ & \\
& & & & & & & $(4.4)$ & & & $(5.2)$ & & & \\
88 & $* * * *$ & $* * * *$ & $* * * *$ & 6.12 & 6.88 & 6.30 & 6.28 & 5.76 & 4.75 & 4.36 & $* * * *$ & $* * * *$ & \\
& & & & $(4.0)$ & $(3.9)$ & $(3.6)$ & $(4.9)$ & $(3.6)$ & $(4.8)$ & $(4.7)$ & & & \\
89 & 3.41 & 4.25 & 5.15 & 6.23 & 6.89 & 6.35 & 6.42 & 5.85 & 5.10 & 3.94 & 3.50 & 3.06 & 5.02 \\
& $(4.5)$ & $(4.1)$ & $(5.5)$ & $(4.2)$ & $(3.6)$ & $(4.0)$ & $(3.6)$ & $(4.0)$ & $(5.4)$ & $(4.1)$ & $(3.8)$ & $(6.0)$ & $(3.5)$ \\
90 & 3.41 & 3.92 & 5.51 & 6.48 & 6.85 & 6.30 & 6.36 & 5.93 & 5.29 & 4.53 & 3.63 & 3.17 & 5.12 \\
& $(3.6)$ & $(5.8)$ & $(4.1)$ & $(3.9)$ & $(3.7)$ & $(3.8)$ & $(4.5)$ & $(4.8)$ & $(3.9)$ & $(3.7)$ & $(4.1)$ & $(6.8)$ & $(3.5)$ \\
91 & $* * * *$ & $* * * *$ & $* * * *$ & 5.43 & 6.32 & 6.14 & 5.73 & 5.86 & 5.42 & 4.18 & 3.22 & 2.77 & \\
& & & & $(4.5)$ & $(3.9)$ & $(4.3)$ & $(4.7)$ & $(4.3)$ & $(4.0)$ & $(4.2)$ & $(4.0)$ & $(3.6)$ & \\
92 & $* * * *$ & 3.41 & 4.83 & 5.85 & 6.76 & 5.85 & $* * * *$ & $* * * *$ & 4.71 & 4.16 & 2.84 & 2.62 & \\
& & $(5.4)$ & $(4.4)$ & $(3.9)$ & $(4.0)$ & $(4.4)$ & & & $(5.5)$ & $(4.9)$ & $(4.9)$ & $(4.6)$ & \\
93 & 2.79 & 3.80 & 4.74 & 6.40 & 5.86 & $* * * *$ & 5.74 & 5.41 & 5.09 & 3.45 & 2.70 & 2.78 & \\
& $(5.2)$ & $(5.1)$ & $(4.9)$ & $(4.3)$ & $(5.3)$ & & $(6.5)$ & $(5.7)$ & $(7.7)$ & $(8.2)$ & $(7.3)$ & $(6.8)$ & \\
\hline \multirow{4}{*}{ Ave } & 3.23 & 3.85 & 5.09 & 6.20 & 6.59 & 6.09 & 6.17 & 5.59 & 5.02 & 4.15 & 3.23 & 2.91 & 4.85 \\
& $(3.7)$ & $(3.9)$ & $(3.8)$ & $(3.5)$ & $(3.5)$ & $(3.6)$ & $(3.7)$ & $(3.6)$ & $(3.8)$ & $(3.6)$ & $(3.7)$ & $(3.8)$ & $(3.4)$ \\
& & & & & & & & & & & & & \\
\hline
\end{tabular}


Table 5-4. Bethune-Cookman College Direct Normal Solar Radiation $\left(\mathbf{k W h} / \mathrm{m}^{2}\right)$ (Percentage Uncertainty Shown in Parenthesis, Asterisks Denote Insufficient Data)

\begin{tabular}{|c|ccccccccccccc|c|}
\hline Year & Jan & Feb & Mar & Apr & May & June & July & Aug & Sept & Oct & Nov & Dec & Ann \\
\hline 85 & & & & & & & & 3.12 & 4.49 & 4.51 & 4.48 & 4.31 & \\
& & & & & & & & $(15.4)$ & $(8.1)$ & $(4.8)$ & $(6.8)$ & $(4.4)$ \\
86 & 4.80 & 4.13 & 5.35 & 7.67 & $* * * *$ & $* * * *$ & $* * * *$ & $* * * *$ & $* * * *$ & $* * * *$ & $* * * *$ & $* * * *$ \\
& $(7.2)$ & $(11.7)$ & $(7.0)$ & $(4.3)$ & & & & & & & & \\
87 & $* * * *$ & $* * * *$ & $* * * *$ & $* * * *$ & $* * * *$ & $* * * *$ & 5.31 & $* * * *$ & $* * * *$ & $* * * *$ & $* * * *$ & $* * * *$ \\
& & & & & & & $(6.0)$ & & & & & \\
88 & $* * * *$ & $* * * *$ & $* * * *$ & 5.42 & 5.96 & 4.33 & 5.09 & 4.26 & $* * * *$ & 4.70 & $* * * *$ & $* * * *$ & \\
& & & & $(5.2)$ & $(5.6)$ & $(4.3)$ & $(7.0)$ & $(6.6)$ & & $(6.7)$ & & & \\
89 & 4.90 & 5.70 & 4.84 & 6.40 & 6.07 & 5.11 & 5.31 & 4.60 & 4.73 & 3.90 & 4.38 & 4.61 & 5.04 \\
& $(7.0)$ & $(6.7)$ & $(8.4)$ & $(8.9)$ & $(4.4)$ & $(4.7)$ & $(4.0)$ & $(6.7)$ & $(8.1)$ & $(7.5)$ & $(4.4)$ & $(7.8)$ & $(4.0)$ \\
90 & 4.57 & 4.73 & 6.04 & 6.36 & 5.70 & 4.87 & 5.39 & 4.75 & 4.63 & 4.65 & 4.89 & 4.72 & 5.11 \\
& $(4.4)$ & $(9.7)$ & $(5.1)$ & $(4.8)$ & $(4.7)$ & $(4.4)$ & $(6.0)$ & $(5.7)$ & $(4.4)$ & $(4.8)$ & $(8.1)$ & $(9.0)$ & $(3.9)$ \\
91 & $* * * *$ & $* * * *$ & $* * * *$ & $* * * *$ & $* * * *$ & 4.91 & $* * * *$ & $* * * *$ & $* * * *$ & $* * * *$ & $* * * *$ & $* * * *$ & \\
& & & & & & $(5.7)$ & & & & & & & \\
92 & $* * * *$ & $* * * *$ & $* * * *$ & $* * * *$ & $* * * *$ & $* * * *$ & $* * * *$ & $* * * *$ & $* * * *$ & $* * * *$ & $* * * *$ & $* * * *$ & \\
& & & & & & & & & & & & & \\
93 & $* * * *$ & $* * * *$ & $* * * *$ & $* * * *$ & $* * * *$ & $* * * *$ & $* * * *$ & $* * * *$ & $* * * *$ & $* * * *$ & $* * * *$ & $* * * *$ & \\
& & & & & & & & & & & & & \\
\hline \multirow{5}{*}{ Ave } & 4.76 & 4.85 & 5.41 & 6.46 & 5.91 & 4.81 & 5.27 & 4.18 & 4.62 & 4.44 & 4.58 & 4.55 & 4.99 \\
& $(4.7)$ & $(6.0)$ & $(4.9)$ & $(4.3)$ & $(4.1)$ & $(3.9)$ & $(4.3)$ & $(5.2)$ & $(5.1)$ & $(4.3)$ & $(4.9)$ & $(5.2)$ & $(3.7)$ \\
\hline
\end{tabular}


Table 5-5. Bluefield State College Global Horizontal Solar Radiation ( $\left.k W h / m^{2}\right)$

(Percentage Uncertainty Shown in Parenthesis, Asterisks Denote Insufficient Data)

\begin{tabular}{|c|c|c|c|c|c|c|c|c|c|c|c|c|c|}
\hline Year & Jan & Feb & Mar & Apr & May & June & July & Aug & Sept & Oct & Nov & Dec & Ann \\
\hline 85 & & & & & & & & & & & $\begin{array}{r}1.66 \\
(14.0)\end{array}$ & $\begin{array}{l}1.60 \\
(8.0)\end{array}$ & \\
\hline 86 & $\begin{array}{r}2.20 \\
(4.9)\end{array}$ & $\begin{array}{c}1.81 \\
(12.1)\end{array}$ & $* * * *$ & $* * * *$ & $\begin{array}{r}4.75 \\
(5.1)\end{array}$ & $\begin{array}{r}6.28 \\
(3.9)\end{array}$ & $\begin{array}{r}5.67 \\
(4.0)\end{array}$ & $\begin{array}{r}4.69 \\
(3.9)\end{array}$ & $\begin{array}{r}4.10 \\
(4.4)\end{array}$ & $\begin{array}{l}3.14 \\
(5.9)\end{array}$ & $\begin{array}{r}1.41 \\
(10.2)\end{array}$ & $\begin{array}{l}1.55 \\
(7.0)\end{array}$ & \\
\hline 87 & $\begin{array}{l}1.43 \\
(8.5)\end{array}$ & $\begin{array}{r}2.36 \\
(8.2)\end{array}$ & $\begin{array}{r}4.02 \\
(4.4)\end{array}$ & $* * * *$ & $\begin{array}{r}5.60 \\
(4.7)\end{array}$ & $\begin{array}{r}5.79 \\
(4.2)\end{array}$ & $* * * *$ & $\begin{array}{r}5.30 \\
(6.1)\end{array}$ & $\begin{array}{r}3.82 \\
(4.3)\end{array}$ & $\begin{array}{r}3.54 \\
(5.3)\end{array}$ & $\begin{array}{r}2.25 \\
(6.0)\end{array}$ & $\begin{array}{l}1.57 \\
(6.0)\end{array}$ & \\
\hline 88 & $\begin{array}{l}2.08 \\
(5.1)\end{array}$ & $\begin{array}{l}3.13 \\
(5.6)\end{array}$ & $\begin{array}{r}4.24 \\
(4.1)\end{array}$ & $\begin{array}{l}5.21 \\
(4.9)\end{array}$ & $* * * *$ & $\begin{array}{r}6.90 \\
(4.8)\end{array}$ & $\begin{array}{r}5.91 \\
(3.8)\end{array}$ & $\begin{array}{r}5.35 \\
(3.9)\end{array}$ & $\begin{array}{r}4.26 \\
(4.2)\end{array}$ & $\begin{array}{r}3.54 \\
(4.7)\end{array}$ & $\begin{array}{r}2.38 \\
(4.8)\end{array}$ & $\begin{array}{l}2.08 \\
(5.3)\end{array}$ & \\
\hline 89 & $\begin{array}{l}2.21 \\
(4.3)\end{array}$ & $\begin{array}{r}2.10 \\
(8.1)\end{array}$ & $\begin{array}{r}3.78 \\
(5.1)\end{array}$ & $\begin{array}{r}4.77 \\
(4.8)\end{array}$ & $\begin{array}{r}4.82 \\
(4.8)\end{array}$ & $\begin{array}{r}5.28 \\
(3.6)\end{array}$ & $\begin{array}{r}5.00 \\
(4.2)\end{array}$ & $\begin{array}{r}4.95 \\
(3.9)\end{array}$ & $\begin{array}{r}3.49 \\
(7.8)\end{array}$ & $\begin{array}{l}3.48 \\
(6.5)\end{array}$ & $\begin{array}{c}2.11 \\
(7.5)\end{array}$ & $\begin{array}{l}1.61 \\
(7.7)\end{array}$ & $\begin{array}{r}3.64 \\
(3.6)\end{array}$ \\
\hline 90 & $\begin{array}{l}1.87 \\
(5.0)\end{array}$ & $\begin{array}{r}2.92 \\
(4.6)\end{array}$ & $\begin{array}{l}3.79 \\
(6.0)\end{array}$ & $\begin{array}{r}4.49 \\
(5.7)\end{array}$ & $\begin{array}{l}5.05 \\
(4.2)\end{array}$ & $\begin{array}{r}6.49 \\
(4.1)\end{array}$ & $\begin{array}{r}5.83 \\
(3.8)\end{array}$ & $\begin{array}{l}5.01 \\
(3.7)\end{array}$ & $\begin{array}{r}4.26 \\
(4.2)\end{array}$ & $\begin{array}{r}3.36 \\
(5.3)\end{array}$ & $\begin{array}{r}2.87 \\
(4.4)\end{array}$ & $\begin{array}{l}1.68 \\
(4.7)\end{array}$ & $\begin{array}{r}3.97 \\
(3.5)\end{array}$ \\
\hline 91 & $\begin{array}{l}1.70 \\
(6.0)\end{array}$ & $\begin{array}{c}2.63 \\
(4.6)\end{array}$ & $\begin{array}{c}3.58 \\
(5.5)\end{array}$ & $\begin{array}{r}4.76 \\
(4.3)\end{array}$ & $\begin{array}{r}5.63 \\
(4.0)\end{array}$ & $\begin{array}{r}5.39 \\
(4.0)\end{array}$ & $\begin{array}{r}5.41 \\
(3.6)\end{array}$ & $\begin{array}{r}4.95 \\
(4.4)\end{array}$ & $\begin{array}{r}3.73 \\
(4.4)\end{array}$ & $\begin{array}{r}3.63 \\
(4.0)\end{array}$ & $\begin{array}{r}2.06 \\
(5.2)\end{array}$ & $\begin{array}{l}1.56 \\
(6.1)\end{array}$ & $\begin{array}{r}3.76 \\
(3.5)\end{array}$ \\
\hline 92 & $\begin{array}{l}2.05 \\
(3.7)\end{array}$ & $\begin{array}{r}2.56 \\
(4.4)\end{array}$ & $\begin{array}{r}3.29 \\
(5.5)\end{array}$ & $\begin{array}{l}4.21 \\
(4.8)\end{array}$ & $\begin{array}{r}4.53 \\
(4.7)\end{array}$ & $* * * *$ & $\begin{array}{r}5.54 \\
(5.2)\end{array}$ & $\begin{array}{r}4.69 \\
(4.4)\end{array}$ & $* * * *$ & $\begin{array}{r}3.52 \\
(4.9)\end{array}$ & $\begin{array}{l}1.78 \\
(5.3)\end{array}$ & $\begin{array}{r}1.30 \\
(11.8)\end{array}$ & \\
\hline 93 & $\begin{array}{r}2.02 \\
(4.9) \\
\end{array}$ & $\begin{array}{r}2.87 \\
(4.8) \\
\end{array}$ & $\begin{array}{l}2.71 \\
(8.0) \\
\end{array}$ & $\begin{array}{r}4.79 \\
(6.2) \\
\end{array}$ & $\begin{array}{r}5.56 \\
(6.2) \\
\end{array}$ & $\begin{array}{r}5.81 \\
(6.9) \\
\end{array}$ & $\begin{array}{r}5.83 \\
(3.8) \\
\end{array}$ & $\begin{array}{r}4.73 \\
(5.3) \\
\end{array}$ & $* * * *$ & $\begin{array}{r}2.46 \\
(11.2) \\
\end{array}$ & $\begin{array}{r}1.88 \\
(9.3) \\
\end{array}$ & $\begin{array}{r}1.28 \\
(9.6) \\
\end{array}$ & \\
\hline Ave & $\begin{array}{l}1.94 \\
(3.7)\end{array}$ & $\begin{array}{r}2.55 \\
(3.9)\end{array}$ & $\begin{array}{l}3.63 \\
(3.7)\end{array}$ & $\begin{array}{r}4.70 \\
(3.8)\end{array}$ & $\begin{array}{l}5.13 \\
(3.6)\end{array}$ & $\begin{array}{r}5.99 \\
(3.6)\end{array}$ & $\begin{array}{l}5.60 \\
(3.5)\end{array}$ & $\begin{array}{r}4.96 \\
(3.6)\end{array}$ & $\begin{array}{r}3.94 \\
(3.7)\end{array}$ & $\begin{array}{r}3.33 \\
(3.8)\end{array}$ & $\begin{array}{c}2.04 \\
(4.0)\end{array}$ & $\begin{array}{r}1.58 \\
(4.0)\end{array}$ & $\begin{array}{r}3.79 \\
(3.4)\end{array}$ \\
\hline
\end{tabular}


Table 5-6. Bluefield State College Direct Normal Solar Radiation $\left(\mathrm{kWh} / \mathrm{m}^{2}\right)$ (Percentage Uncertainty Shown in Parenthesis, Asterisks Denote Insufficient Data)

\begin{tabular}{|c|c|c|c|c|c|c|c|c|c|c|c|c|c|}
\hline Year & Jan & Feb & Mar & Apr & May & June & July & Aug & Sept & Oct & Nov & Dec & Ann \\
\hline 85 & & & & & & & & & & & $\begin{array}{r}1.62 \\
(38.0)\end{array}$ & $\begin{array}{r}1.90 \\
(21.9)\end{array}$ & \\
\hline 86 & $\begin{array}{r}3.49 \\
(4.5)\end{array}$ & $\begin{array}{c}1.21 \\
(36.2)\end{array}$ & $* * * *$ & $* * * *$ & $\begin{array}{r}3.16 \\
(10.9)\end{array}$ & $\begin{array}{r}4.67 \\
(3.7)\end{array}$ & $\begin{array}{r}3.34 \\
(4.8)\end{array}$ & $\begin{array}{r}3.30 \\
(5.1)\end{array}$ & $\begin{array}{l}3.09 \\
(8.5)\end{array}$ & $\begin{array}{r}3.21 \\
(11.0)\end{array}$ & $\begin{array}{r}1.16 \\
(27.3)\end{array}$ & $\begin{array}{r}2.17 \\
(13.4)\end{array}$ & \\
\hline 87 & $\begin{array}{r}1.49 \\
(17.9)\end{array}$ & $\begin{array}{r}2.34 \\
(17.2)\end{array}$ & $\begin{array}{r}4.30 \\
(3.7)\end{array}$ & $* * * *$ & $\begin{array}{r}4.20 \\
(6.0)\end{array}$ & $\begin{array}{l}3.85 \\
(5.8)\end{array}$ & $* * * *$ & $\begin{array}{r}3.70 \\
(10.3)\end{array}$ & $\begin{array}{r}3.16 \\
(7.2)\end{array}$ & $\begin{array}{r}5.04 \\
(4.8)\end{array}$ & $\begin{array}{r}2.97 \\
(9.9)\end{array}$ & $\begin{array}{r}2.17 \\
(10.7)\end{array}$ & \\
\hline 88 & $\begin{array}{r}2.58 \\
(7.9)\end{array}$ & $\begin{array}{r}3.67 \\
(7.5)\end{array}$ & $\begin{array}{r}4.24 \\
(2.9)\end{array}$ & $\begin{array}{r}4.69 \\
(4.2)\end{array}$ & $* * * *$ & $\begin{array}{r}5.59 \\
(4.4)\end{array}$ & $\begin{array}{r}4.12 \\
(5.0)\end{array}$ & $\begin{array}{r}4.07 \\
(3.0)\end{array}$ & $\begin{array}{r}3.96 \\
(4.4)\end{array}$ & $\begin{array}{r}4.42 \\
(3.2)\end{array}$ & $\begin{array}{c}3.28 \\
(5.1)\end{array}$ & $\begin{array}{c}3.48 \\
(5.4)\end{array}$ & \\
\hline 89 & $\begin{array}{l}3.19 \\
(3.3)\end{array}$ & $\begin{array}{r}2.32 \\
(13.0)\end{array}$ & $\begin{array}{r}3.81 \\
(5.9)\end{array}$ & $\begin{array}{r}3.66 \\
(5.1)\end{array}$ & $\begin{array}{l}3.16 \\
(7.8)\end{array}$ & $\begin{array}{l}3.06 \\
(4.8)\end{array}$ & $\begin{array}{r}2.51 \\
(9.4)\end{array}$ & $\begin{array}{r}2.99 \\
(4.3)\end{array}$ & $\begin{array}{r}2.42 \\
(21.8)\end{array}$ & $\begin{array}{r}4.57 \\
(6.8)\end{array}$ & $\begin{array}{c}2.98 \\
(12.2)\end{array}$ & $\begin{array}{r}1.94 \\
(19.0)\end{array}$ & $\begin{array}{l}3.06 \\
(3.2)\end{array}$ \\
\hline 90 & $\begin{array}{r}2.25 \\
(8.3)\end{array}$ & $\begin{array}{r}3.33 \\
(7.2)\end{array}$ & $\begin{array}{r}3.71 \\
(8.0)\end{array}$ & $\begin{array}{r}3.83 \\
(9.0)\end{array}$ & $\begin{array}{r}3.29 \\
(4.5)\end{array}$ & $\begin{array}{r}4.81 \\
(3.6)\end{array}$ & $\begin{array}{r}3.78 \\
(4.2)\end{array}$ & $\begin{array}{r}2.98 \\
(4.3)\end{array}$ & $\begin{array}{c}3.28 \\
(5.7)\end{array}$ & $\begin{array}{r}4.52 \\
(4.4)\end{array}$ & $\begin{array}{r}4.86 \\
(3.2)\end{array}$ & $\begin{array}{r}2.46 \\
(5.9)\end{array}$ & $\begin{array}{r}3.59 \\
(2.4)\end{array}$ \\
\hline 91 & $\begin{array}{r}2.05 \\
(7.7)\end{array}$ & $\begin{array}{r}3.23 \\
(5.4)\end{array}$ & $\begin{array}{r}3.30 \\
(6.3)\end{array}$ & $\begin{array}{r}4.20 \\
(4.2)\end{array}$ & $\begin{array}{r}4.03 \\
(4.7)\end{array}$ & $\begin{array}{r}3.02 \\
(6.7)\end{array}$ & $\begin{array}{r}2.91 \\
(1.8)\end{array}$ & $\begin{array}{l}3.24 \\
(7.2)\end{array}$ & $\begin{array}{r}2.70 \\
(7.3)\end{array}$ & $\begin{array}{r}4.07 \\
(2.8)\end{array}$ & $\begin{array}{r}2.35 \\
(8.1)\end{array}$ & $\begin{array}{r}2.02 \\
(10.5)\end{array}$ & $\begin{array}{l}3.09 \\
(2.4)\end{array}$ \\
\hline 92 & $\begin{array}{r}2.57 \\
(4.3)\end{array}$ & $\begin{array}{r}2.40 \\
(5.8)\end{array}$ & $\begin{array}{r}2.55 \\
(7.4)\end{array}$ & $\begin{array}{r}2.61 \\
(7.9)\end{array}$ & $\begin{array}{c}2.98 \\
(7.0)\end{array}$ & $* * * *$ & $\begin{array}{r}3.28 \\
(13.9)\end{array}$ & $\begin{array}{r}2.42 \\
(9.3)\end{array}$ & $* * *$ & $\begin{array}{r}4.30 \\
(4.8)\end{array}$ & $\begin{array}{l}1.99 \\
(8.3)\end{array}$ & $\begin{array}{r}0.96 \\
(50.0)\end{array}$ & \\
\hline 93 & $\begin{array}{r}2.32 \\
(9.1) \\
\end{array}$ & $\begin{array}{r}3.50 \\
(7.3) \\
\end{array}$ & $\begin{array}{r}1.79 \\
(17.9) \\
\end{array}$ & $\begin{array}{r}3.57 \\
(10.6) \\
\end{array}$ & $\begin{array}{r}3.72 \\
(12.1) \\
\end{array}$ & $\begin{array}{r}3.62 \\
(17.8) \\
\end{array}$ & $\begin{array}{r}3.87 \\
(6.2) \\
\end{array}$ & $\begin{array}{r}2.51 \\
(14.3) \\
\end{array}$ & $* * * *$ & $\begin{array}{r}2.20 \\
(29.7) \\
\end{array}$ & $\begin{array}{r}2.11 \\
(21.3) \\
\end{array}$ & $\begin{array}{r}1.21 \\
(34.2) \\
\end{array}$ & \\
\hline Ave & $\begin{array}{r}2.49 \\
(3.1)\end{array}$ & $\begin{array}{r}2.75 \\
(4.1)\end{array}$ & $\begin{array}{r}3.39 \\
(3.0)\end{array}$ & $\begin{array}{r}3.76 \\
(3.3)\end{array}$ & $\begin{array}{r}3.51 \\
(3.4)\end{array}$ & $\begin{array}{r}4.09 \\
(3.4)\end{array}$ & $\begin{array}{r}3.40 \\
(3.3)\end{array}$ & $\begin{array}{l}3.15 \\
(3.2)\end{array}$ & $\begin{array}{r}3.10 \\
(4.2)\end{array}$ & $\begin{array}{r}4.04 \\
(3.3)\end{array}$ & $\begin{array}{r}2.59 \\
(4.6)\end{array}$ & $\begin{array}{r}2.03 \\
(5.6)\end{array}$ & $\begin{array}{l}3.19 \\
(2.1)\end{array}$ \\
\hline
\end{tabular}


Table 5-7. Elizabeth City State University Global Horizontal Solar Radiation $\left(\mathrm{kWh} / \mathrm{m}^{2}\right)$ (Percentage Uncertainty Shown in Parenthesis, Asterisks Denote Insufficient Data)

\begin{tabular}{|c|c|c|c|c|c|c|c|c|c|c|c|c|c|}
\hline Year & Jan & Feb & Mar & Apr & May & June & July & Aug & Sept & Oct & Nov & Dec & Ann \\
\hline 85 & & & & & & & & & $\begin{array}{l}5.02 \\
(8.1)\end{array}$ & * & $* * * *$ & $* *=$ & \multirow{9}{*}{$\begin{array}{r}4.22 \\
(3.5)\end{array}$} \\
\hline 86 & $\begin{array}{c}2.54 \\
(7.1)\end{array}$ & $\begin{array}{c}2.47 \\
(8.2)\end{array}$ & $\begin{array}{r}4.51 \\
(4.4)\end{array}$ & $\begin{array}{r}5.13 \\
(3.9)\end{array}$ & $\begin{array}{r}5.86 \\
(5.8)\end{array}$ & $\begin{array}{r}6.50 \\
(4.0)\end{array}$ & $* * * *$ & $* * * *$ & $\begin{array}{r}4.61 \\
(7.1)\end{array}$ & $* * * *$ & $\begin{array}{l}1.95 \\
(6.6)\end{array}$ & $* * * *$ & \\
\hline 87 & $\begin{array}{r}2.36 \\
(6.2)\end{array}$ & $\begin{array}{r}3.00 \\
(4.4)\end{array}$ & $\begin{array}{r}4.01 \\
(4.2)\end{array}$ & $\begin{array}{r}4.43 \\
(4.2)\end{array}$ & $\begin{array}{c}5.98 \\
(4.2)\end{array}$ & $\begin{array}{r}6.08 \\
(3.6)\end{array}$ & $\begin{array}{l}6.15 \\
(3.5)\end{array}$ & $\begin{array}{r}5.36 \\
(4.7)\end{array}$ & $\begin{array}{r}4.24 \\
(3.7)\end{array}$ & $\begin{array}{r}4.29 \\
(7.5)\end{array}$ & $\begin{array}{c}2.65 \\
(5.3)\end{array}$ & $\begin{array}{c}2.03 \\
(4.8)\end{array}$ & \\
\hline 88 & $\begin{array}{c}2.15 \\
(5.0)\end{array}$ & $* * * *$ & $* * * *$ & $* * * *$ & $* * * *$ & $* * * *$ & $* * * *$ & $* * * *$ & $* * * *$ & $* * * *$ & $* * * *$ & $* * * *$ & \\
\hline 89 & $* * * *$ & $* * * *$ & $* * * *$ & $* * * *$ & $\begin{array}{c}6.64 \\
(5.6)\end{array}$ & $\begin{array}{r}6.47 \\
(3.8)\end{array}$ & $\begin{array}{r}5.80 \\
(3.7)\end{array}$ & $\begin{array}{r}5.13 \\
(4.9)\end{array}$ & $\begin{array}{r}4.04 \\
(4.5)\end{array}$ & $\begin{array}{r}3.67 \\
(4.0)\end{array}$ & $\begin{array}{l}2.79 \\
(4.0)\end{array}$ & $\begin{array}{c}2.23 \\
(6.1)\end{array}$ & \\
\hline 90 & $\begin{array}{l}2.48 \\
(4.0)\end{array}$ & $\begin{array}{r}3.42 \\
(5.0)\end{array}$ & $\begin{array}{r}4.24 \\
(4.8)\end{array}$ & $\begin{array}{r}5.80 \\
(4.2)\end{array}$ & $\begin{array}{r}5.80 \\
(4.6)\end{array}$ & $\begin{array}{l}7.32 \\
(7.5)\end{array}$ & $* * * *$ & $\begin{array}{l}5.31 \\
(5.8)\end{array}$ & $\begin{array}{r}4.84 \\
(4.9)\end{array}$ & $\begin{array}{r}4.01 \\
(5.4)\end{array}$ & $\begin{array}{r}2.99 \\
(5.4)\end{array}$ & $\begin{array}{l}1.97 \\
(4.0)\end{array}$ & \\
\hline 91 & $\begin{array}{r}2.08 \\
(4.8)\end{array}$ & $\begin{array}{r}2.93 \\
(4.7)\end{array}$ & $\begin{array}{c}3.91 \\
(4.6)\end{array}$ & $\begin{array}{c}5.31 \\
(6.1)\end{array}$ & $\begin{array}{r}6.06 \\
(6.4)\end{array}$ & $* * * *$ & $\begin{array}{c}5.78 \\
(4.3)\end{array}$ & $\begin{array}{l}5.05 \\
(5.4)\end{array}$ & $\begin{array}{r}4.91 \\
(4.0)\end{array}$ & $\begin{array}{c}3.62 \\
(4.9)\end{array}$ & $\begin{array}{l}2.72 \\
(4.0)\end{array}$ & $\begin{array}{l}2.31 \\
(4.0)\end{array}$ & \\
\hline 92 & $\begin{array}{c}2.24 \\
(5.0)\end{array}$ & $\begin{array}{c}2.94 \\
(5.2)\end{array}$ & $\begin{array}{r}4.34 \\
(3.9)\end{array}$ & $\begin{array}{r}5.43 \\
(3.7)\end{array}$ & $\begin{array}{r}4.95 \\
(3.9)\end{array}$ & $* * * *$ & $\begin{array}{r}6.00 \\
(3.5)\end{array}$ & $\begin{array}{r}4.92 \\
(7.4)\end{array}$ & $\begin{array}{c}4.39 \\
(3.9)\end{array}$ & $\begin{array}{c}3.58 \\
(4.0)\end{array}$ & $\begin{array}{r}2.42 \\
(4.2)\end{array}$ & $\begin{array}{l}1.65 \\
(4.2)\end{array}$ & \\
\hline 93 & $\begin{array}{r}2.10 \\
(4.2) \\
\end{array}$ & $\begin{array}{r}2.96 \\
(6.7) \\
\end{array}$ & $\begin{array}{r}3.72 \\
(7.5) \\
\end{array}$ & $\begin{array}{r}5.45 \\
(4.0) \\
\end{array}$ & $* * * *$ & $\begin{array}{r}6.12 \\
(4.7) \\
\end{array}$ & $\begin{array}{r}5.81 \\
(4.3) \\
\end{array}$ & $\begin{array}{r}5.14 \\
(5.3) \\
\end{array}$ & $\begin{array}{r}4.51 \\
(4.6) \\
\end{array}$ & $\begin{array}{r}2.87 \\
(6.5) \\
\end{array}$ & $\begin{array}{r}2.38 \\
(5.9) \\
\end{array}$ & $\begin{array}{r}1.77 \\
(6.1) \\
\end{array}$ & \\
\hline Ave & $\begin{array}{c}2.28 \\
(3.7)\end{array}$ & $\begin{array}{r}2.95 \\
(3.9)\end{array}$ & $\begin{array}{r}4.12 \\
(3.7)\end{array}$ & $\begin{array}{c}5.26 \\
(3.6)\end{array}$ & $\begin{array}{c}5.88 \\
(3.8)\end{array}$ & $\begin{array}{r}6.50 \\
(3.8)\end{array}$ & $\begin{array}{c}5.91 \\
(3.5)\end{array}$ & $\begin{array}{l}5.15 \\
(3.9)\end{array}$ & $\begin{array}{r}4.57 \\
(3.7)\end{array}$ & $\begin{array}{c}3.67 \\
(3.9)\end{array}$ & $\begin{array}{r}2.56 \\
(3.7)\end{array}$ & $\begin{array}{l}1.99 \\
(3.7)\end{array}$ & $\begin{array}{c}4.24 \\
(3.4)\end{array}$ \\
\hline
\end{tabular}


Table 5-8. Elizabeth City State University Direct Normal Solar Radiation (kWh/m²) (Percentage Uncertainty Shown in Parenthesis, Asterisks Denote Insufficient Data)

\begin{tabular}{|c|rrrrrrrrrrrr|r|r|}
\hline Year & Jan & Feb & Mar & Apr & May & June & July & Aug & Sept & Oct & Nov & Dec & Ann \\
\hline \multirow{2}{*}{85} & & & & & & & & & $* * * *$ & $* * * *$ & $* * * *$ & $* * * *$ & \\
& & & & & & & & & & & & & \\
86 & 4.13 & 2.11 & 4.84 & 4.84 & 4.27 & 4.78 & $* * * *$ & $* * * *$ & 4.14 & $* * * *$ & 1.94 & $* * * * *$ & \\
& $(8.8)$ & $(28.7)$ & $(5.1)$ & $(4.4)$ & $(9.4)$ & $(4.8)$ & & & $(9.2)$ & & $(17.0)$ & & \\
87 & 3.63 & 3.69 & 4.44 & 3.77 & 4.80 & 4.52 & 4.38 & 3.36 & 3.31 & 5.80 & 3.83 & 3.14 & 4.06 \\
& $(9.2)$ & $(6.4)$ & $(5.0)$ & $(6.2)$ & $(5.2)$ & $(4.5)$ & $(4.1)$ & $(7.6)$ & $(4.6)$ & $(8.8)$ & $(6.9)$ & $(6.9)$ & $(3.9)$ \\
88 & 3.01 & $* * * *$ & $* * * *$ & $* * * *$ & $* * * *$ & $* * * *$ & $* * * *$ & $* * * *$ & $* * * *$ & $* * * *$ & $* * * *$ & $* * * *$ & \\
& $(8.9)$ & & & & & & & & & & & & \\
89 & $* * * *$ & $* * * *$ & $* * * *$ & $* * * *$ & 5.85 & 4.70 & 3.40 & 3.25 & 2.91 & 4.65 & 4.31 & 3.85 & \\
& & & & & $(6.7)$ & $(4.4)$ & $(6.3)$ & $(8.5)$ & $(8.6)$ & $(4.4)$ & $(4.4)$ & $(7.0)$ & \\
90 & 4.14 & 4.46 & 4.11 & 5.17 & 4.22 & 5.79 & $* * * *$ & 3.10 & 3.83 & 4.84 & 4.73 & 2.70 & \\
& $(4.5)$ & $(6.8)$ & $(5.9)$ & $(3.6)$ & $(5.6)$ & $(9.8)$ & & $(10.3)$ & $(5.7)$ & $(5.1)$ & $(5.5)$ & $(3.8)$ & \\
91 & 2.82 & 3.44 & 3.61 & 4.17 & 4.38 & $* * * *$ & 3.02 & 2.78 & 4.17 & 3.89 & 3.42 & 3.34 & \\
& $(6.6)$ & $(7.0)$ & $(6.0)$ & $(8.3)$ & $(9.6)$ & & $(8.9)$ & $(10.6)$ & $(3.2)$ & $(5.4)$ & $(4.0)$ & $(3.8)$ & \\
92 & 2.56 & 2.85 & 3.60 & 4.07 & 2.88 & $* * * *$ & 3.79 & 3.17 & 3.42 & 3.93 & 2.59 & 1.70 & \\
& $(10.5)$ & $(11.1)$ & $(4.4)$ & $(3.1)$ & $(5.1)$ & & $(1.8)$ & $(15.0)$ & $(3.2)$ & $(2.9)$ & $(4.3)$ & $(6.6)$ & \\
93 & 2.35 & 3.32 & 2.87 & 4.98 & $* * * *$ & 4.21 & 3.63 & 3.52 & 3.98 & 2.76 & 3.04 & 2.32 & \\
& $(5.1)$ & $(13.0)$ & $(19.1)$ & $(2.6)$ & & $(6.6)$ & $(7.8)$ & $(8.0)$ & $(4.9)$ & $(12.0)$ & $(10.0)$ & $(12.6)$ & \\
\hline \multirow{4}{*}{ Ave } & 3.23 & 3.31 & 3.91 & 4.50 & 4.40 & 4.80 & 3.64 & 3.20 & 3.68 & 4.31 & 3.41 & 2.84 & 3.77 \\
& $(4.0)$ & $(5.2)$ & $(3.8)$ & $(2.9)$ & $(3.8)$ & $(4.0)$ & $(3.4)$ & $(4.7)$ & $(3.3)$ & $(3.5)$ & $(3.6)$ & $(3.6)$ & $(2.7)$ \\
& & & & & & & & & & & & & \\
\hline
\end{tabular}


Table 5-9. Mississippi Valley State University Global Horizontal Solar Radiation $\left(\mathrm{kWh} / \mathrm{m}^{2}\right)$ (Percentage Uncertainty Shown in Parenthesis, Asterisks Denote Insufficient Data)

\begin{tabular}{|c|cccccccccccc|c|}
\hline Year & Jan & Feb & Mar & Apr & May & June & July & Aug & Sept & Oct & Nov & Dec & Ann \\
\hline \multirow{5}{*}{85} & & & & & & & 6.39 & 5.79 & 5.03 & 3.33 & 2.41 & 2.66 & \\
& & & & & & & $(3.5)$ & $(3.7)$ & $(3.6)$ & $(4.4)$ & $(4.0)$ & $(5.0)$ & \\
86 & 3.01 & 3.23 & 5.34 & 5.83 & 5.56 & 6.27 & 6.67 & 6.04 & 4.88 & 3.62 & 1.95 & 1.84 & 4.53 \\
& $(3.7)$ & $(5.1)$ & $(4.2)$ & $(3.7)$ & $(4.3)$ & $(3.6)$ & $(3.5)$ & $(3.5)$ & $(3.4)$ & $(4.9)$ & $(7.7)$ & $(6.9)$ & $(3.5)$ \\
87 & 2.59 & 2.97 & 4.86 & 6.26 & 6.01 & 6.60 & 6.49 & 5.93 & 5.21 & 4.52 & 3.07 & 2.35 & 4.75 \\
& $(5.9)$ & $(7.0)$ & $(4.8)$ & $(3.7)$ & $(4.5)$ & $(3.8)$ & $(3.6)$ & $(3.7)$ & $(3.7)$ & $(3.4)$ & $(4.3)$ & $(5.0)$ & $(3.5)$ \\
88 & 2.64 & $* * * *$ & 4.90 & 6.28 & 7.14 & 7.06 & 6.00 & 5.95 & 4.72 & 3.72 & 2.95 & 2.63 & \\
& $(4.8)$ & & $(5.7)$ & $(4.6)$ & $(4.1)$ & $(3.4)$ & $(3.8)$ & $(3.5)$ & $(4.6)$ & $(4.5)$ & $(4.0)$ & $(4.8)$ & \\
89 & 2.60 & 2.77 & 3.76 & 5.80 & 6.07 & 5.46 & 5.78 & 5.91 & 4.52 & 4.04 & $* * * *$ & 2.70 & \\
& $(6.9)$ & $(7.6)$ & $(6.2)$ & $(4.6)$ & $(4.8)$ & $(4.0)$ & $(3.5)$ & $(3.4)$ & $(4.8)$ & $(4.3)$ & & $(6.3)$ & \\
90 & 2.73 & 3.31 & 4.18 & 5.84 & 6.00 & 6.91 & 6.47 & 6.30 & 5.15 & 4.21 & 3.16 & 2.10 & 4.70 \\
& $(6.6)$ & $(4.5)$ & $(5.7)$ & $(5.3)$ & $(4.8)$ & $(4.2)$ & $(3.5)$ & $(3.7)$ & $(3.7)$ & $(4.0)$ & $(4.3)$ & $(6.3)$ & $(3.5)$ \\
91 & 2.18 & 3.27 & 4.41 & 4.87 & 5.42 & 6.15 & 6.63 & 5.90 & 5.40 & 4.05 & 2.88 & 2.14 & 4.45 \\
& $(6.7)$ & $(6.1)$ & $(5.5)$ & $(6.7)$ & $(4.4)$ & $(3.6)$ & $(3.7)$ & $(3.7)$ & $(3.9)$ & $(3.7)$ & $(4.0)$ & $(5.5)$ & $(3.5)$ \\
92 & 2.71 & 3.62 & 4.32 & 5.92 & 6.26 & 5.80 & 6.23 & 5.70 & $* * * *$ & 4.64 & 2.71 & 1.74 & \\
& $(5.7)$ & $(5.8)$ & $(4.5)$ & $(3.7)$ & $(3.8)$ & $(5.4)$ & $(5.1)$ & $(3.5)$ & & $(7.6)$ & $(5.2)$ & $(10.9)$ & \\
93 & 2.12 & 2.96 & 3.73 & 4.83 & 6.09 & 6.14 & 6.65 & 5.63 & 5.18 & 3.53 & 2.46 & 2.10 & 4.29 \\
& $(6.8)$ & $(4.4)$ & $(6.3)$ & $(4.8)$ & $(3.8)$ & $(3.6)$ & $(3.5)$ & $(3.7)$ & $(3.9)$ & $(4.2)$ & $(4.9)$ & $(6.0)$ & $(3.5)$ \\
\hline \multirow{5}{*}{ Ave } & 2.57 & 3.16 & 4.44 & 5.70 & 6.07 & 6.30 & 6.37 & 5.91 & 5.01 & 3.96 & 2.70 & 2.25 & 4.54 \\
& $(3.8)$ & $(3.8)$ & $(3.7)$ & $(3.6)$ & $(3.5)$ & $(3.5)$ & $(3.4)$ & $(3.4)$ & $(3.5)$ & $(3.6)$ & $(3.6)$ & $(3.8)$ & $(3.4)$ \\
& & & & & & & & & & & & & \\
\hline
\end{tabular}


Table 5-10. Mississippi Valley State University Direct Normal Solar Radiation $\left(\mathrm{kWh} / \mathrm{m}^{2}\right)$ (Percentage Uncertainty Shown in Parenthesis, Asterisks Denote Insufficient Data)

\begin{tabular}{|c|cccccccccccc|c|}
\hline Year & Jan & Feb & Mar & Apr & May & June & July & Aug & Sept & Oct & Nov & Dec & Ann \\
\hline \multirow{4}{*}{85} & & & & & & & 4.92 & 4.62 & 5.07 & 3.42 & 2.45 & 4.39 & \\
& & & & & & & $(4.3)$ & $(4.8)$ & $(4.0)$ & $(7.8)$ & $(7.1)$ & $(6.0)$ & \\
86 & 4.68 & 3.66 & 6.32 & 5.27 & 3.71 & 4.66 & 5.35 & 5.17 & 4.29 & 4.30 & 1.38 & 1.95 & 4.24 \\
& $(4.0)$ & $(10.3)$ & $(4.8)$ & $(4.1)$ & $(8.4)$ & $(5.1)$ & $(4.0)$ & $(4.0)$ & $(3.6)$ & $(6.6)$ & $(27.5)$ & $(19.6)$ & $(3.9)$ \\
87 & 3.50 & 3.16 & 6.53 & 6.51 & 4.04 & 5.28 & 4.50 & 4.42 & 5.29 & 6.70 & 4.60 & 3.35 & 4.83 \\
& $(10.5)$ & $(17.5)$ & $(8.4)$ & $(4.0)$ & $(8.1)$ & $(4.6)$ & $(4.6)$ & $(5.0)$ & $(5.7)$ & $(5.7)$ & $(5.7)$ & $(7.9)$ & $(4.0)$ \\
88 & 3.87 & $* * * *$ & 6.18 & 6.37 & 7.04 & 6.00 & 4.08 & 4.84 & 4.17 & 4.96 & 4.12 & 4.64 & \\
& $(9.2)$ & & $(8.4)$ & $(5.4)$ & $(5.1)$ & $(4.0)$ & $(6.4)$ & $(4.4)$ & $(8.9)$ & $(7.0)$ & $(5.5)$ & $(7.0)$ & \\
89 & 3.82 & $* * * *$ & $* * * *$ & 6.00 & 5.31 & 3.38 & 3.70 & 4.54 & 4.34 & 5.26 & $* * * *$ & 4.51 & \\
& $(11.0)$ & & & $(8.1)$ & $(9.0)$ & $(13.9)$ & $(6.7)$ & $(4.5)$ & $(10.4)$ & $(6.2)$ & & $(8.0)$ & \\
90 & 4.02 & 3.75 & 3.97 & 5.42 & 4.50 & 6.15 & 4.85 & 5.07 & 4.62 & 5.73 & 5.17 & 2.64 & 4.66 \\
& $(8.2)$ & $(6.6)$ & $(10.3)$ & $(6.0)$ & $(7.9)$ & $(5.1)$ & $(4.0)$ & $(4.4)$ & $(5.1)$ & $(5.1)$ & $(5.7)$ & $(14.6)$ & $(3.9)$ \\
91 & 2.55 & 4.08 & 4.35 & 3.90 & 3.45 & 4.28 & 5.38 & 4.30 & 5.18 & 4.49 & 3.10 & 2.30 & 3.94 \\
& $(14.8)$ & $(9.4)$ & $(9.3)$ & $(13.5)$ & $(10.0)$ & $(4.7)$ & $(4.3)$ & $(4.6)$ & $(4.4)$ & $(4.1)$ & $(5.6)$ & $(15.2)$ & $(4.2)$ \\
92 & 3.31 & 4.26 & 4.04 & 4.82 & 4.39 & 3.78 & 4.27 & 3.80 & $* * * *$ & 5.71 & 3.56 & $* * * *$ & \\
& $(9.1)$ & $(9.1)$ & $(9.7)$ & $(4.8)$ & $(5.1)$ & $(13.9)$ & $(10.2)$ & $(4.4)$ & & $(8.8)$ & $(9.4)$ & & \\
93 & 2.36 & 2.90 & 3.05 & 3.87 & 4.65 & 4.20 & 5.38 & 3.77 & 5.56 & 3.89 & 3.18 & 3.19 & 3.84 \\
& $(15.5)$ & $(6.1)$ & $(13.3)$ & $(4.9)$ & $(3.0)$ & $(3.5)$ & $(2.5)$ & $(3.2)$ & $(3.1)$ & $(4.7)$ & $(6.8)$ & $(9.6)$ & $(2.4)$ \\
\hline \multirow{4}{*}{ Ave } & 3.51 & 3.63 & 4.92 & 5.27 & 4.64 & 4.72 & 4.71 & 4.50 & 4.82 & 4.94 & 3.45 & 3.37 & 4.38 \\
& $(4.7)$ & $(5.1)$ & $(4.5)$ & $(3.9)$ & $(4.0)$ & $(4.0)$ & $(3.7)$ & $(3.5)$ & $(3.8)$ & $(3.9)$ & $(4.2)$ & $(4.7)$ & $(3.4)$ \\
& & & & & & & & & & & & & \\
\end{tabular}


Table 5-11. South Carolina State University Global Horizontal Solar Radiation $\left(\mathbf{k W h} / \mathrm{m}^{2}\right)$ (Percentage Uncertainty Shown in Parenthesis, Asterisks Denote Insufficient Data)

\begin{tabular}{|c|cccccccccccc|c|}
\hline Year & Jan & Feb & Mar & Apr & May & June & July & Aug & Sept & Oct & Nov & Dec & Ann \\
\hline \multirow{2}{*}{85} & & & & & & & & 5.23 & 5.39 & 3.26 & $* * * *$ & 2.87 & \\
& & & & & & & & $(4.8)$ & $(3.4)$ & $(4.3)$ & & $(7.9)$ & \\
86 & 2.88 & 3.36 & 4.78 & 6.65 & 6.29 & 6.21 & 6.37 & 4.90 & 4.49 & $* * * *$ & 2.19 & 2.36 & \\
& $(4.9)$ & $(4.6)$ & $(3.9)$ & $(5.3)$ & $(5.1)$ & $(4.0)$ & $(4.1)$ & $(3.7)$ & $(4.8)$ & & $(10.4)$ & $(4.3)$ & \\
87 & 2.63 & 3.09 & 4.46 & 6.12 & 6.46 & 6.06 & $* * * *$ & 5.50 & 4.43 & 4.67 & 3.07 & 2.43 & \\
& $(4.8)$ & $(6.0)$ & $(5.1)$ & $(4.3)$ & $(5.7)$ & $(5.4)$ & & $(7.3)$ & $(4.3)$ & $(4.0)$ & $(4.1)$ & $(4.3)$ & \\
88 & $* * * *$ & $* * * *$ & $* * * *$ & 5.70 & 6.64 & 6.97 & 6.06 & 5.26 & 4.13 & 4.29 & 3.02 & 2.68 & \\
& & & & $(4.0)$ & $(3.7)$ & $(4.3)$ & $(3.7)$ & $(4.3)$ & $(4.2)$ & $(4.0)$ & $(3.6)$ & $(3.9)$ & \\
89 & 2.45 & 3.11 & 3.83 & 5.69 & 6.44 & 6.19 & 5.67 & 4.82 & 3.87 & 3.89 & 2.99 & 2.49 & 4.29 \\
& $(4.4)$ & $(4.8)$ & $(5.7)$ & $(4.6)$ & $(3.4)$ & $(4.0)$ & $(4.3)$ & $(3.7)$ & $(4.5)$ & $(3.7)$ & $(4.2)$ & $(5.3)$ & $(3.5)$ \\
90 & 2.91 & 3.25 & 4.23 & 6.12 & 6.26 & 6.79 & 6.20 & 5.31 & 5.14 & $* * * *$ & 3.41 & 2.19 & \\
& $(3.7)$ & $(5.9)$ & $(4.4)$ & $(3.9)$ & $(4.0)$ & $(4.0)$ & $(3.4)$ & $(3.7)$ & $(3.7)$ & & $(4.3)$ & $(4.8)$ & \\
91 & 2.11 & 3.48 & $* * * *$ & $* * * *$ & 5.12 & 6.27 & 5.49 & 4.86 & 5.14 & 4.05 & 3.04 & 2.55 & \\
& $(7.0)$ & $(4.4)$ & & & $(4.1)$ & $(3.7)$ & $(5.5)$ & $(3.7)$ & $(3.7)$ & $(3.7)$ & $(4.4)$ & $(4.4)$ & \\
92 & 2.48 & 3.10 & 4.62 & 5.40 & 5.88 & 5.52 & 6.22 & 4.82 & 4.56 & 3.85 & 2.72 & 2.14 & 4.28 \\
& $(4.2)$ & $(4.6)$ & $(4.5)$ & $(4.0)$ & $(3.7)$ & $(5.8)$ & $(4.1)$ & $(3.7)$ & $(3.7)$ & $(3.7)$ & $(4.8)$ & $(4.6)$ & $(3.5)$ \\
93 & 2.22 & 3.21 & 4.32 & 6.07 & 6.33 & 6.35 & 6.60 & 5.79 & 4.94 & 3.20 & 3.08 & 2.59 & 4.56 \\
& $(6.0)$ & $(4.4)$ & $(5.6)$ & $(4.3)$ & $(5.7)$ & $(4.3)$ & $(4.2)$ & $(4.3)$ & $(4.8)$ & $(4.4)$ & $(4.3)$ & $(4.6)$ & $(3.5)$ \\
\hline \multirow{4}{*}{ Ave } & 2.53 & 3.23 & 4.37 & 5.96 & 6.18 & 6.30 & 6.09 & 5.17 & 4.68 & 3.89 & 2.94 & 2.48 & 4.49 \\
& $(3.7)$ & $(3.7)$ & $(3.7)$ & $(3.6)$ & $(3.6)$ & $(3.5)$ & $(3.5)$ & $(3.6)$ & $(3.5)$ & $(3.5)$ & $(3.6)$ & $(3.6)$ & $(3.4)$ \\
& & & & & & & & & & & & & \\
\hline
\end{tabular}


Table 5-12. South Carolina State University Direct Normal Solar Radiation $\left(\mathrm{kWh} / \mathrm{m}^{2}\right)$ (Percentage Uncertainty Shown in Parenthesis, Asterisks Denote Insufficient Data)

\begin{tabular}{|c|rccccccccccc|c|}
\hline Year & Jan & Feb & Mar & Apr & May & June & July & Aug & Sept & Oct & Nov & Dec & Ann \\
\hline \multirow{5}{*}{85} & & & & & & & & 3.69 & 5.58 & 2.94 & $* * * *$ & 5.04 & \\
& & & & & & & & $(6.8)$ & $(3.6)$ & $(8.8)$ & & $(9.8)$ & \\
86 & 4.61 & 3.49 & 5.20 & 7.27 & 4.91 & 4.13 & 4.46 & 3.16 & 3.75 & $* * * *$ & $* * * *$ & 3.08 & \\
& $(5.7)$ & $(9.9)$ & $(4.4)$ & $(6.6)$ & $(6.5)$ & $(5.2)$ & $(5.0)$ & $(4.9)$ & $(10.8)$ & & & $(6.7)$ & \\
87 & 4.00 & $* * * *$ & $* * * *$ & 5.71 & 4.95 & 3.91 & $* * * *$ & 3.59 & 3.67 & 6.56 & 4.45 & 3.72 & \\
& $(6.7)$ & & & $(7.5)$ & $(8.3)$ & $(11.7)$ & & $(12.8)$ & $(9.7)$ & $(5.4)$ & $(6.0)$ & $(7.3)$ & \\
88 & $* * * *$ & $* * * *$ & $* * * *$ & 5.75 & 5.52 & 5.61 & 4.02 & 3.99 & $* * * *$ & 5.60 & 4.25 & 4.45 & \\
& & & & $(7.7)$ & $(5.4)$ & $(6.0)$ & $(7.7)$ & $(9.9)$ & & $(5.4)$ & $(6.0)$ & $(4.8)$ & \\
89 & 3.77 & 4.18 & $* * * *$ & $* * * *$ & 5.51 & 4.44 & 3.72 & 3.39 & $* * * *$ & 5.03 & 4.26 & 4.33 & \\
& $(10.6)$ & $(12.3)$ & & & $(6.0)$ & $(7.1)$ & $(10.6)$ & $(14.6)$ & & $(7.4)$ & $(6.8)$ & $(8.4)$ & \\
90 & 4.52 & 4.23 & $* * * *$ & 5.85 & 5.10 & 5.09 & 4.36 & 3.23 & 4.67 & $* * * *$ & 5.80 & 2.65 & \\
& $(4.0)$ & $(12.4)$ & & $(10.3)$ & $(9.9)$ & $(4.4)$ & $(3.6)$ & $(4.8)$ & $(4.4)$ & & $(5.4)$ & $(10.1)$ & \\
91 & 2.48 & 4.96 & $* * * *$ & $* * * *$ & 2.76 & 4.35 & 3.22 & 2.93 & 4.84 & 4.61 & 3.96 & 3.73 & \\
& $(14.2)$ & $(8.8)$ & & & $(15.4)$ & $(4.3)$ & $(12.0)$ & $(5.2)$ & $(5.4)$ & $(4.9)$ & $(5.8)$ & $(5.4)$ & \\
92 & 2.91 & 2.76 & 4.45 & 4.49 & 4.48 & 3.02 & 4.13 & 2.81 & 3.58 & 4.11 & $* * * *$ & 2.46 & \\
& $(9.1)$ & $(19.5)$ & $(9.6)$ & $(11.5)$ & $(8.7)$ & $(16.3)$ & $(5.4)$ & $(5.3)$ & $(4.6)$ & $(4.9)$ & & $(10.4)$ & \\
93 & 2.12 & 3.23 & $* * * *$ & 5.98 & $* * * *$ & $* * * *$ & 4.72 & 3.74 & $* * * *$ & $* * * *$ & 4.23 & 3.94 & \\
& $(18.2)$ & $(17.1)$ & & $(8.9)$ & & & $(7.2)$ & $(14.0)$ & & & $(7.9)$ & $(6.3)$ & \\
\hline \multirow{4}{*}{ Ave } & 3.49 & 3.81 & 4.82 & 5.84 & 4.75 & 4.36 & 4.09 & 3.39 & 4.35 & 4.81 & 4.49 & 3.71 & 4.33 \\
& $(4.7)$ & $(6.2)$ & $(5.6)$ & $(4.8)$ & $(4.5)$ & $(4.4)$ & $(4.3)$ & $(4.8)$ & $(4.2)$ & $(4.1)$ & $(4.2)$ & $(4.3)$ & $(3.7)$ \\
& & & & & & & & & & & & & \\
\hline
\end{tabular}


Table 5-13. Savannah State College Global Horizontal Solar Radiation (kWh/m $\left.{ }^{2}\right)$

(Percentage Uncertainty Shown in Parenthesis, Asterisks Denote Insufficient Data)

\begin{tabular}{|c|c|c|c|c|c|c|c|c|c|c|c|c|c|}
\hline Year & Jan & Feb & Mar & Apr & May & June & July & Aug & Sept & Oct & Nov & Dec & Ann \\
\hline 85 & & & & & & & & & $\begin{array}{l}5.14 \\
(3.4)\end{array}$ & $\begin{array}{r}3.26 \\
(6.5)\end{array}$ & $\begin{array}{r}2.78 \\
(4.4)\end{array}$ & $\begin{array}{r}2.93 \\
(4.4)\end{array}$ & \\
\hline 86 & $\begin{array}{c}2.91 \\
(4.5)\end{array}$ & $\begin{array}{l}3.72 \\
(5.2)\end{array}$ & $\begin{array}{l}5.02 \\
(4.7)\end{array}$ & $\begin{array}{l}6.56 \\
(3.6)\end{array}$ & $\begin{array}{l}6.44 \\
(3.8)\end{array}$ & $\begin{array}{l}5.99 \\
(5.7)\end{array}$ & $\begin{array}{r}6.10 \\
(5.9)\end{array}$ & $\begin{array}{l}5.04 \\
(4.3)\end{array}$ & $\begin{array}{c}4.44 \\
(3.4)\end{array}$ & $\begin{array}{r}3.60 \\
(4.5)\end{array}$ & $\begin{array}{r}2.33 \\
(5.9)\end{array}$ & $\begin{array}{r}2.06 \\
(4.9)\end{array}$ & $\begin{array}{r}4.52 \\
(3.5)\end{array}$ \\
\hline 87 & $\begin{array}{c}2.58 \\
(5.3)\end{array}$ & $\begin{array}{c}2.97 \\
(7.5)\end{array}$ & $\begin{array}{r}4.48 \\
(5.1)\end{array}$ & $\begin{array}{r}6.24 \\
(3.6)\end{array}$ & $\begin{array}{l}6.36 \\
(3.8)\end{array}$ & $\begin{array}{l}5.94 \\
(4.5)\end{array}$ & $\begin{array}{c}6.55 \\
(3.8)\end{array}$ & $\begin{array}{l}5.62 \\
(3.7)\end{array}$ & $\begin{array}{r}4.25 \\
(3.9)\end{array}$ & $\begin{array}{r}4.55 \\
(3.9)\end{array}$ & $\begin{array}{l}3.14 \\
(4.3)\end{array}$ & $\begin{array}{r}2.72 \\
(3.9)\end{array}$ & $\begin{array}{r}4.63 \\
(3.5)\end{array}$ \\
\hline 88 & $\begin{array}{c}2.58 \\
(4.9)\end{array}$ & $\begin{array}{r}3.85 \\
(5.2)\end{array}$ & $\begin{array}{c}5.24 \\
(5.3)\end{array}$ & $\begin{array}{r}5.53 \\
(4.5)\end{array}$ & $\begin{array}{l}6.76 \\
(4.4)\end{array}$ & $\begin{array}{l}6.65 \\
(3.9)\end{array}$ & $\begin{array}{r}6.16 \\
(3.6)\end{array}$ & $\begin{array}{r}5.29 \\
(3.5)\end{array}$ & $\begin{array}{r}4.19 \\
(3.9)\end{array}$ & $\begin{array}{r}4.39 \\
(3.6)\end{array}$ & $\begin{array}{l}3.14 \\
(4.0)\end{array}$ & $\begin{array}{r}2.82 \\
(3.7)\end{array}$ & $\begin{array}{r}4.72 \\
(3.5)\end{array}$ \\
\hline 89 & $\begin{array}{c}2.82 \\
(3.4)\end{array}$ & $\begin{array}{r}3.42 \\
(4.1)\end{array}$ & $\begin{array}{r}4.53 \\
(5.2)\end{array}$ & $\begin{array}{r}5.96 \\
(4.6)\end{array}$ & $\begin{array}{l}6.48 \\
(4.0)\end{array}$ & $\begin{array}{r}5.89 \\
(4.5)\end{array}$ & $\begin{array}{r}6.17 \\
(6.1)\end{array}$ & $\begin{array}{r}4.65 \\
(4.3)\end{array}$ & $\begin{array}{r}3.63 \\
(5.3)\end{array}$ & $\begin{array}{c}3.81 \\
(6.6)\end{array}$ & $\begin{array}{c}3.07 \\
(4.2)\end{array}$ & $\begin{array}{r}2.55 \\
(5.2)\end{array}$ & $\begin{array}{r}4.42 \\
(3.6)\end{array}$ \\
\hline 90 & $\begin{array}{c}3.11 \\
(5.9)\end{array}$ & $\begin{array}{r}3.54 \\
(6.5)\end{array}$ & $\begin{array}{c}4.51 \\
(4.2)\end{array}$ & $\begin{array}{l}6.01 \\
(4.0)\end{array}$ & $\begin{array}{r}6.46 \\
(3.6)\end{array}$ & $\begin{array}{l}6.42 \\
(3.7)\end{array}$ & $\begin{array}{r}5.90 \\
(3.7)\end{array}$ & $\begin{array}{l}5.42 \\
(4.0)\end{array}$ & $\begin{array}{c}5.11 \\
(3.7)\end{array}$ & $\begin{array}{r}4.07 \\
(4.4)\end{array}$ & $\begin{array}{r}3.36 \\
(4.3)\end{array}$ & $\begin{array}{r}2.14 \\
(3.7)\end{array}$ & $\begin{array}{r}4.68 \\
(3.5)\end{array}$ \\
\hline 91 & $\begin{array}{r}2.09 \\
(5.9)\end{array}$ & $\begin{array}{r}3.60 \\
(3.9)\end{array}$ & $\begin{array}{r}4.55 \\
(4.6)\end{array}$ & $\begin{array}{r}4.79 \\
(4.6)\end{array}$ & $\begin{array}{l}5.12 \\
(4.2)\end{array}$ & $\begin{array}{r}5.66 \\
(4.5)\end{array}$ & $\begin{array}{r}5.51 \\
(4.3)\end{array}$ & $\begin{array}{r}5.06 \\
(4.0)\end{array}$ & $\begin{array}{r}4.48 \\
(3.4)\end{array}$ & $\begin{array}{c}3.91 \\
(3.6)\end{array}$ & $\begin{array}{r}2.82 \\
(4.2)\end{array}$ & $\begin{array}{r}2.48 \\
(4.1)\end{array}$ & $\begin{array}{r}4.17 \\
(3.5)\end{array}$ \\
\hline 92 & $\begin{array}{r}2.42 \\
(4.7)\end{array}$ & $\begin{array}{l}3.07 \\
(5.2)\end{array}$ & $\begin{array}{r}4.63 \\
(6.8)\end{array}$ & $\begin{array}{l}5.43 \\
(4.6)\end{array}$ & $\begin{array}{l}5.78 \\
(4.2)\end{array}$ & $\begin{array}{r}5.36 \\
(7.4)\end{array}$ & $* * * *$ & $\begin{array}{r}4.76 \\
(4.1)\end{array}$ & $\begin{array}{r}4.12 \\
(3.9)\end{array}$ & $\begin{array}{l}3.96 \\
(4.2)\end{array}$ & $\begin{array}{r}2.73 \\
(6.2)\end{array}$ & $\begin{array}{c}2.26 \\
(3.6)\end{array}$ & \\
\hline 93 & $\begin{array}{r}2.13 \\
(7.6) \\
\end{array}$ & $\begin{array}{r}3.22 \\
(6.4) \\
\end{array}$ & $\begin{array}{r}4.64 \\
(4.9) \\
\end{array}$ & $\begin{array}{l}6.17 \\
(4.0) \\
\end{array}$ & $\begin{array}{r}6.33 \\
(5.4) \\
\end{array}$ & $\begin{array}{r}6.40 \\
(6.1) \\
\end{array}$ & $\begin{array}{r}6.33 \\
(3.9) \\
\end{array}$ & $\begin{array}{r}5.47 \\
(3.7) \\
\end{array}$ & $\begin{array}{c}5.04 \\
(4.0) \\
\end{array}$ & $\begin{array}{r}3.28 \\
(5.0) \\
\end{array}$ & $\begin{array}{r}3.18 \\
(5.0) \\
\end{array}$ & $* * * *$ & \\
\hline Ave & $\begin{array}{r}2.58 \\
(3.7)\end{array}$ & $\begin{array}{c}3.42 \\
(3.7)\end{array}$ & $\begin{array}{r}4.70 \\
(3.7)\end{array}$ & $\begin{array}{l}5.84 \\
(3.5)\end{array}$ & $\begin{array}{r}6.22 \\
(3.5)\end{array}$ & $\begin{array}{r}6.04 \\
(3.7)\end{array}$ & $\begin{array}{c}6.10 \\
(3.6)\end{array}$ & $\begin{array}{r}5.16 \\
(3.5)\end{array}$ & $\begin{array}{r}4.49 \\
(3.5)\end{array}$ & $\begin{array}{l}3.87 \\
(3.6)\end{array}$ & $\begin{array}{r}2.95 \\
(3.6)\end{array}$ & $\begin{array}{r}2.50 \\
(3.5)\end{array}$ & $\begin{array}{r}4.49 \\
(3.4)\end{array}$ \\
\hline
\end{tabular}


Table 5-14. Savannah State College Direct Normal Solar Radiation $\left(\mathrm{kWh} / \mathrm{m}^{2}\right)$ (Percentage Uncertainty Shown in Parenthesis, Asterisks Denote Insufficient Data)

\begin{tabular}{|c|rrrrrrrrrrrr|r}
\hline Year & Jan & Feb & Mar & Apr & May & June & July & Aug & Sept & Oct & Nov & Dec & Ann \\
\hline \multirow{2}{*}{85} & & & & & & & & $* * * *$ & 5.05 & 3.28 & 3.57 & 5.05 & \\
& & & & & & & & & $(6.0)$ & $(17.4)$ & $(10.7)$ & $(5.7)$ & \\
86 & 4.35 & 4.65 & 5.48 & 7.02 & 5.26 & 3.83 & $* * * *$ & 3.97 & 3.41 & 4.64 & 2.12 & 2.37 & \\
& $(5.1)$ & $(7.5)$ & $(6.1)$ & $(4.0)$ & $(4.9)$ & $(9.1)$ & & $(7.6)$ & $(8.8)$ & $(10.5)$ & $(25.9)$ & $(11.6)$ & \\
87 & 4.10 & $* * * *$ & 5.38 & 6.40 & 5.57 & 4.19 & 5.20 & 3.77 & 3.15 & 5.94 & 3.90 & 3.76 & \\
& $(8.5)$ & & $(9.9)$ & $(4.0)$ & $(5.2)$ & $(6.0)$ & $(4.3)$ & $(5.0)$ & $(5.8)$ & $(4.4)$ & $(5.0)$ & $(4.5)$ & \\
88 & 3.40 & 5.28 & 6.06 & 4.96 & $* * * *$ & $* * * *$ & $* * * *$ & $* * * *$ & $* * * *$ & $* * * *$ & $* * * *$ & 4.62 & \\
& $(10.9)$ & $(8.2)$ & $(6.8)$ & $(7.5)$ & & & & & & & & $(4.0)$ & \\
89 & 3.67 & 4.05 & 4.82 & 5.65 & 5.30 & 4.19 & 4.07 & $* * * *$ & 2.60 & 3.75 & 4.09 & 4.03 & \\
& $(3.6)$ & $(8.2)$ & $(8.7)$ & $(6.0)$ & $(4.6)$ & $(6.3)$ & $(12.9)$ & & $(21.7)$ & $(14.2)$ & $(4.8)$ & $(6.5)$ & \\
90 & 4.91 & 4.63 & 5.57 & 6.47 & 5.74 & 4.61 & 4.16 & 3.50 & 4.43 & 4.88 & 5.42 & 2.62 & 4.74 \\
& $(8.0)$ & $(10.4)$ & $(8.9)$ & $(6.3)$ & $(6.4)$ & $(4.8)$ & $(8.7)$ & $(9.7)$ & $(8.1)$ & $(6.7)$ & $(5.4)$ & $(16.1)$ & $(4.1)$ \\
91 & 2.21 & 4.86 & 4.94 & 3.80 & 3.74 & 4.26 & 3.65 & 3.28 & 3.27 & 4.34 & 3.47 & 3.29 & 3.75 \\
& $(17.7)$ & $(7.7)$ & $(8.9)$ & $(14.8)$ & $(14.0)$ & $(8.2)$ & $(10.4)$ & $(11.6)$ & $(9.3)$ & $(7.6)$ & $(12.0)$ & $(7.1)$ & $(4.6)$ \\
92 & 2.88 & $* * * *$ & $* * * *$ & 4.69 & 4.91 & 3.10 & $* * * *$ & 2.90 & 2.89 & 3.94 & 3.05 & 2.61 & \\
& $(14.8)$ & & & $(10.8)$ & $(9.2)$ & $(17.8)$ & & $(8.6)$ & $(6.3)$ & $(6.2)$ & $(11.7)$ & $(5.1)$ & \\
93 & 1.96 & 3.13 & 4.40 & 5.94 & 4.60 & 4.77 & 4.80 & 3.87 & 4.81 & 2.78 & 4.40 & $* * * *$ & \\
& $(22.3)$ & $(14.4)$ & $(7.7)$ & $(4.8)$ & $(8.2)$ & $(7.7)$ & $(4.9)$ & $(4.5)$ & $(4.4)$ & $(11.2)$ & $(5.7)$ & & \\
\hline \multirow{4}{*}{ Ave } & 3.43 & 4.43 & 5.24 & 5.62 & 5.02 & 4.14 & 4.38 & 3.55 & 3.70 & 4.19 & 3.75 & 3.54 & 4.25 \\
& $(5.0)$ & $(5.0)$ & $(4.6)$ & $(4.2)$ & $(4.3)$ & $(4.6)$ & $(4.9)$ & $(4.6)$ & $(4.6)$ & $(4.8)$ & $(4.7)$ & $(4.2)$ & $(3.7)$ \\
\hline
\end{tabular}




\subsection{Monthly Variability}

Monthly variability for global horizontal radiation and direct normal radiation is shown in Figures 5-1 through 5-12. The figures show the ratio of radiation for a particular month to the average radiation for that month recorded over the period of record. Values greater than 1 indicate that more radiation than average was received. For months with more than $30 \%$ of the data missing or failing quality assessment, ratios were not calculated and plotted. Direct normal radiation shows the largest monthly variability. The variations were generally within $\pm 40 \%$, compared to $\pm 20 \%$ for global horizontal radiation.

The eruption of Mt. Pinatubo in the Philippines in June 1991 spewed large amounts of dust into the atmosphere that increased the scattering and absorption of radiation and reduced the amount of radiation reaching the earth. It generally requires a few years before all the volcanic dust falls out of the atmosphere and the effects are no longer seen. This trend is observed in the HBCU data, with lower monthly averages for up to about 2 years after the eruption, and then a return to more normal levels. As expected, direct normal radiation was affected more than global horizontal radiation.

A major El Niño event occurred at about the same time that the Mt. Pinatubo effects would be observed, so some of the reduction in the solar resource might be attributed to the El Niño effect. El Niño results from large bodies of warm water in the Pacific that modify weather patterns, causing some regions to have increased cloudiness and other regions to have decreased cloudiness. For regions with an increase in cloudiness, the amount of solar radiation is reduced.

A way to study just the effects of Mt. Pinatubo would be to look primarily at data recorded under clear skies. This approach by Michalsky et al. (1994) showed peak reductions of direct normal radiation of from $15 \%$ to $20 \%$ for three locations in the United States. 


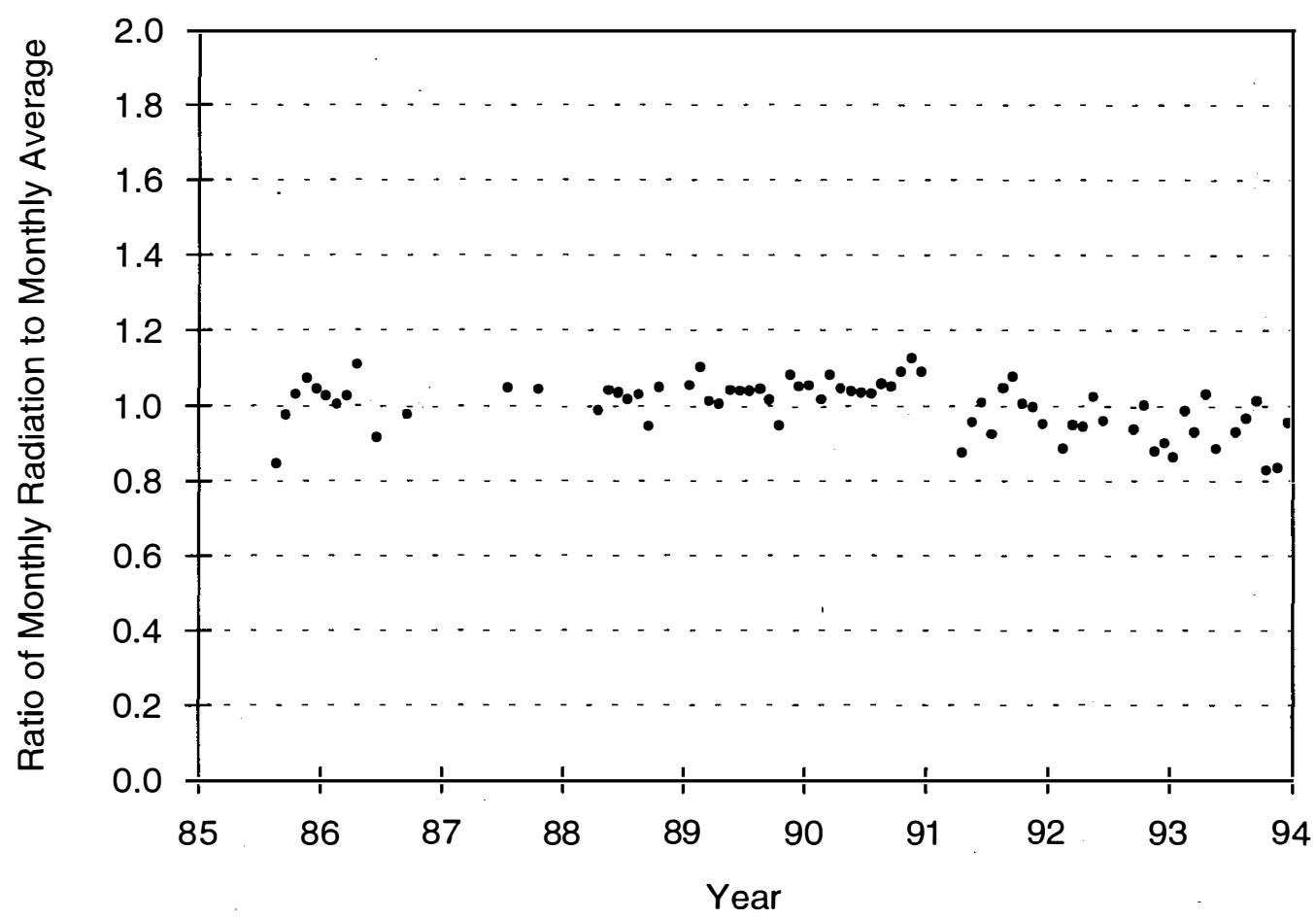

Figure 5-1. Monthly variability of global horizontal radiation for Bethune-Cookman College

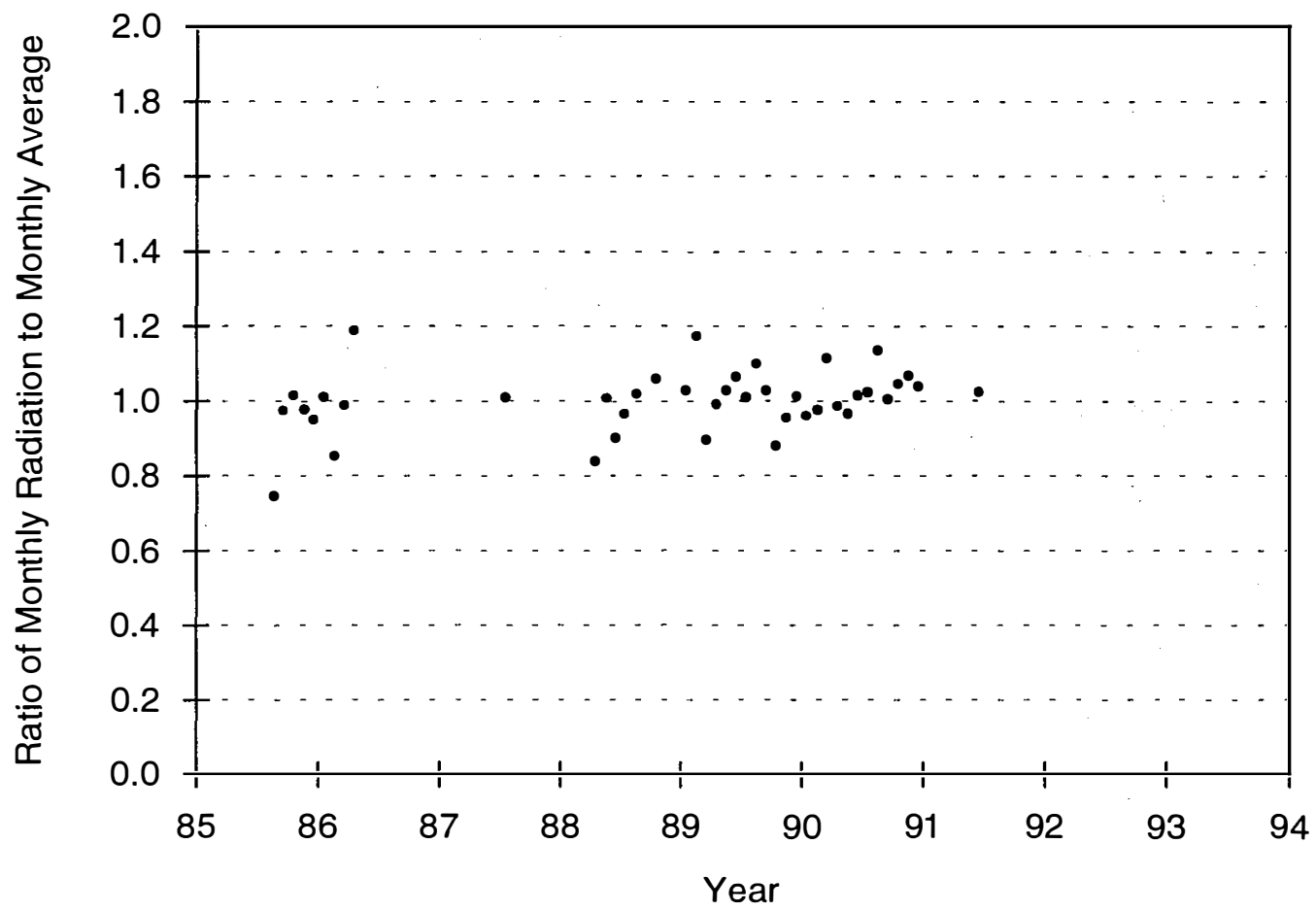

Figure 5-2. Monthly variability of direct normal radiation for Bethune-Cookman College 


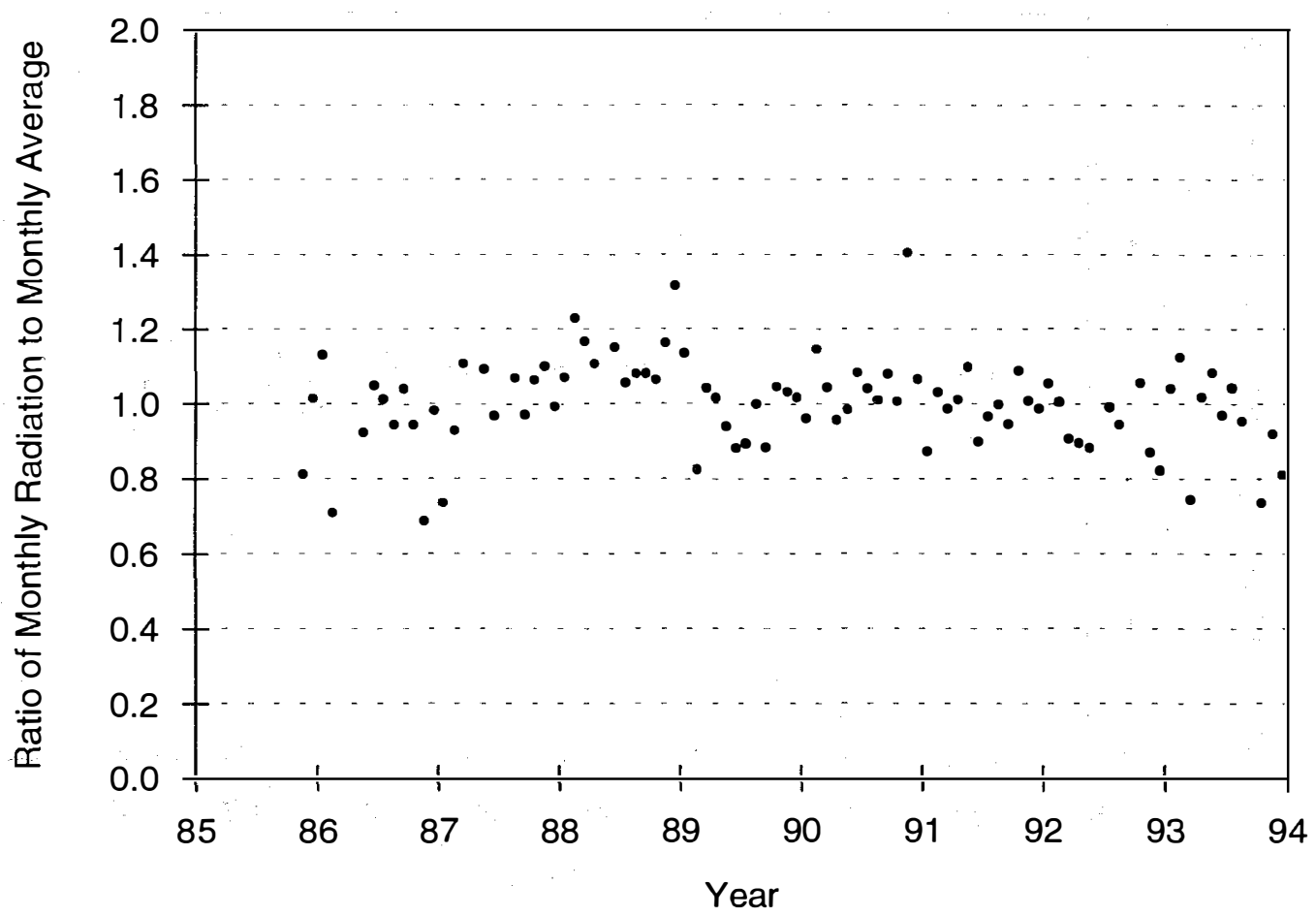

Figure 5-3. Monthly variability of global horizontal radiation for Bluefield State College

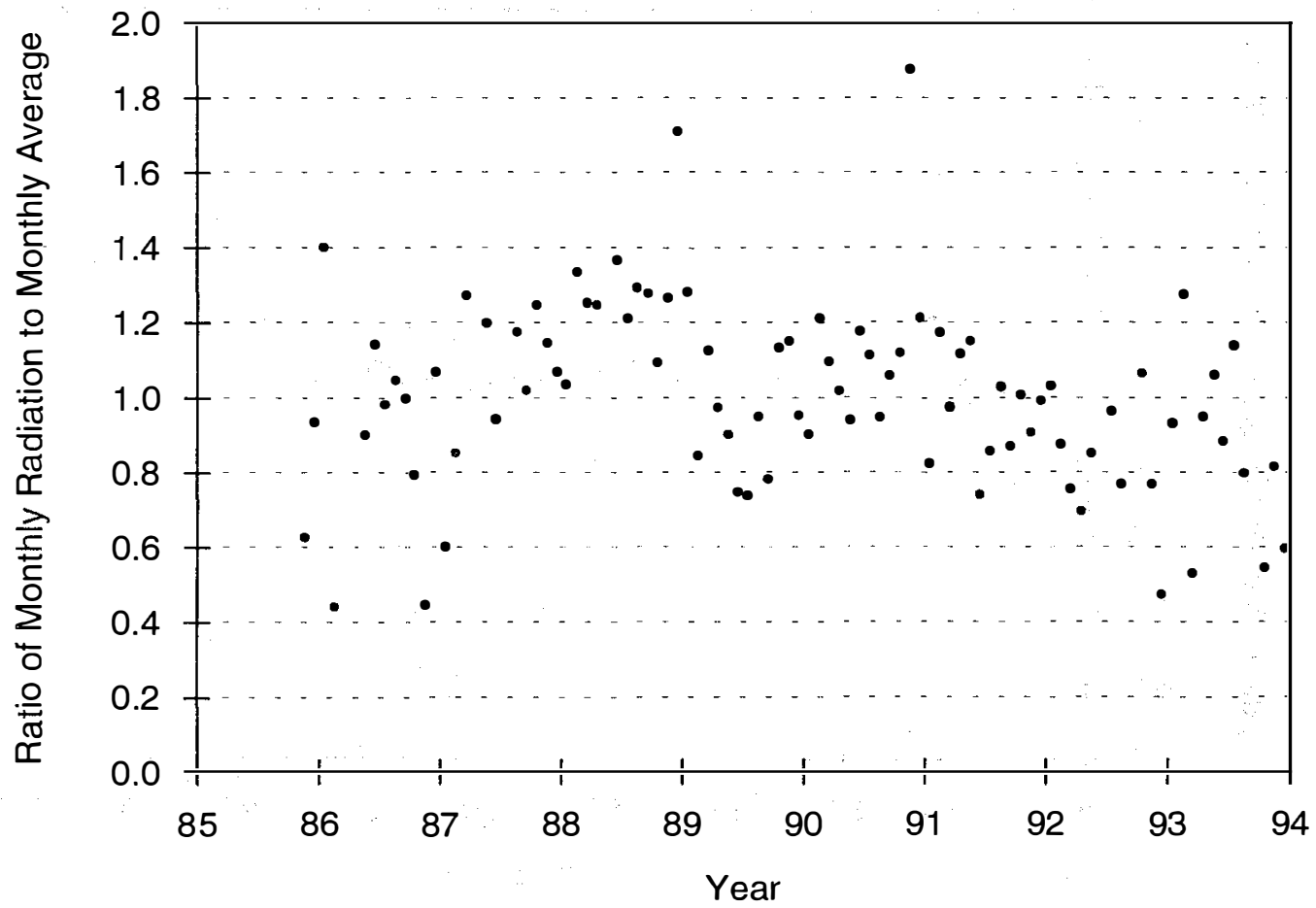

Figure 5-4. Monthly variability of direct normal radiation for Bluefield State College 


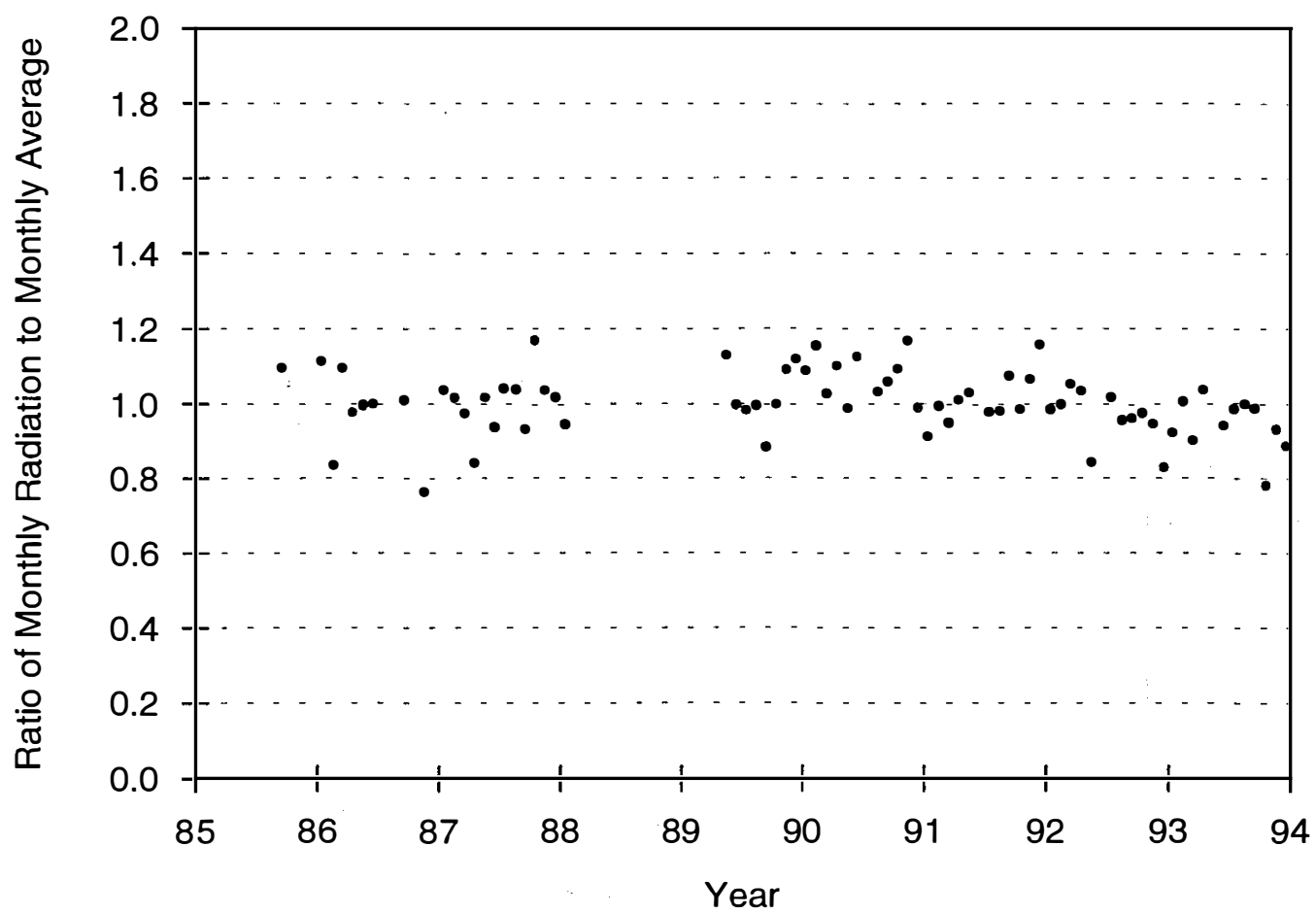

Figure 5-5. Monthly variability of global horizontal radiation for Elizabeth City State College

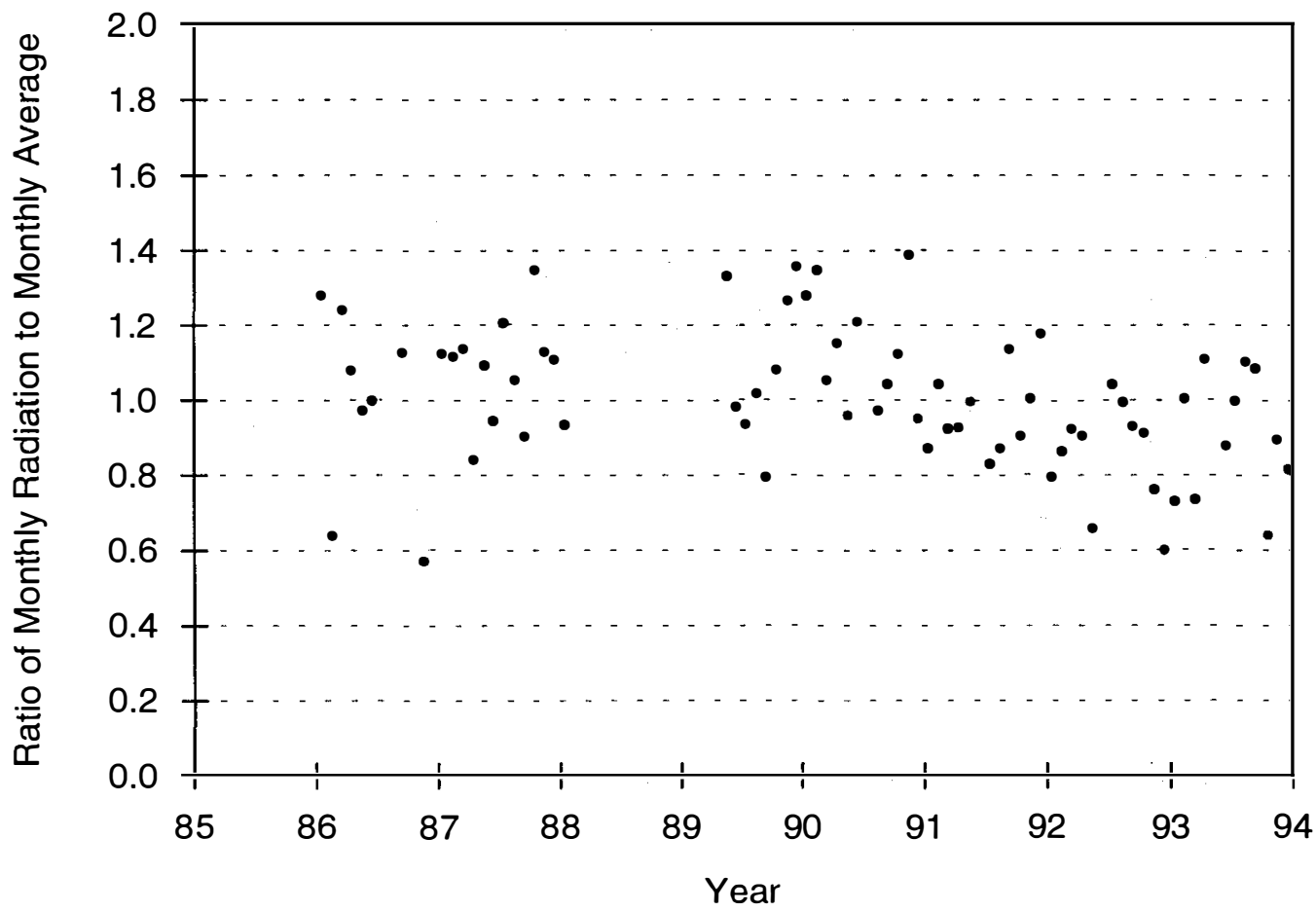

Figure 5-6. Monthly variability of direct normal radiation for Elizabeth City State College 


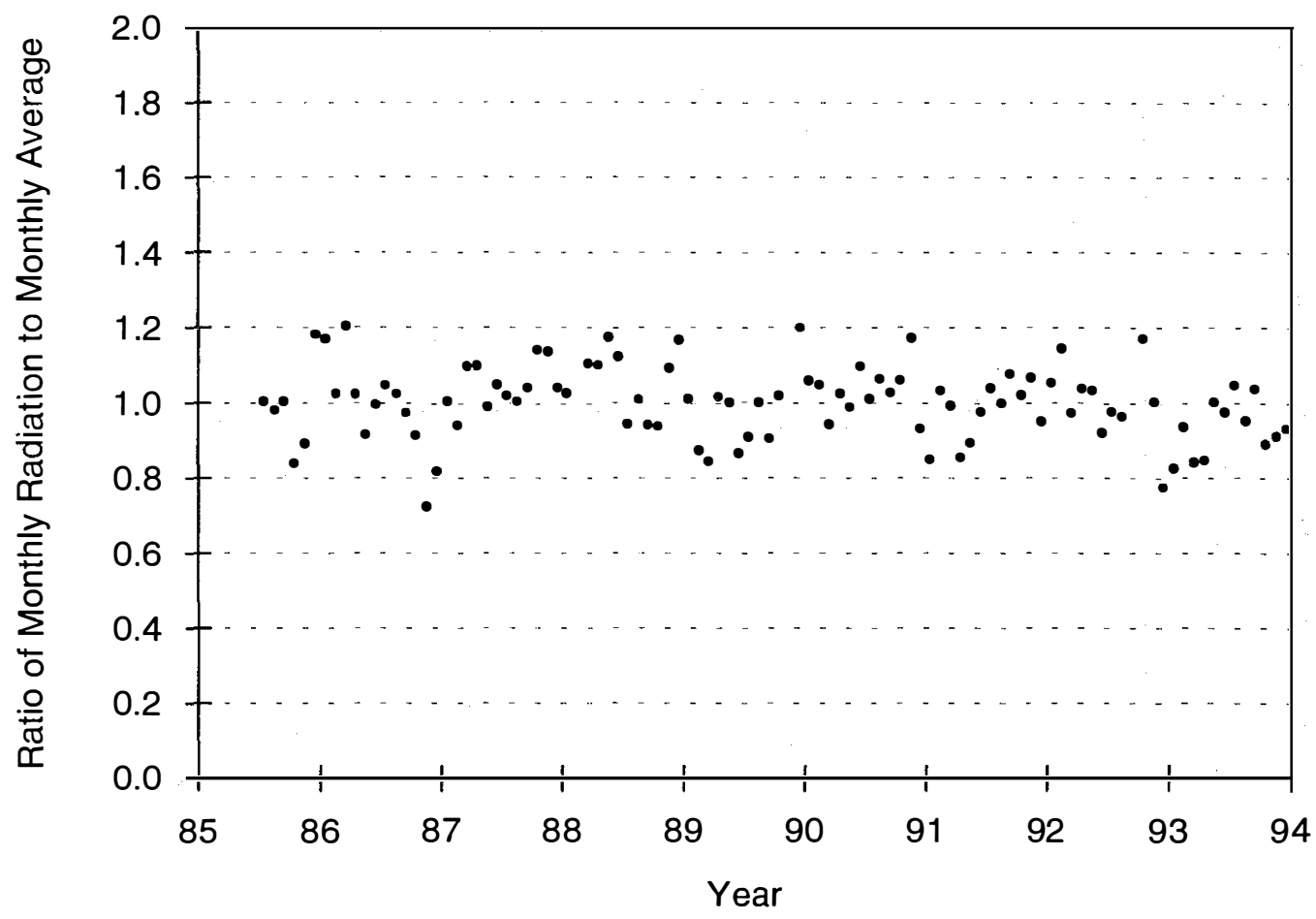

Figure 5-7. Monthly variability of global horizontal radiation for Mississippi Valley State University

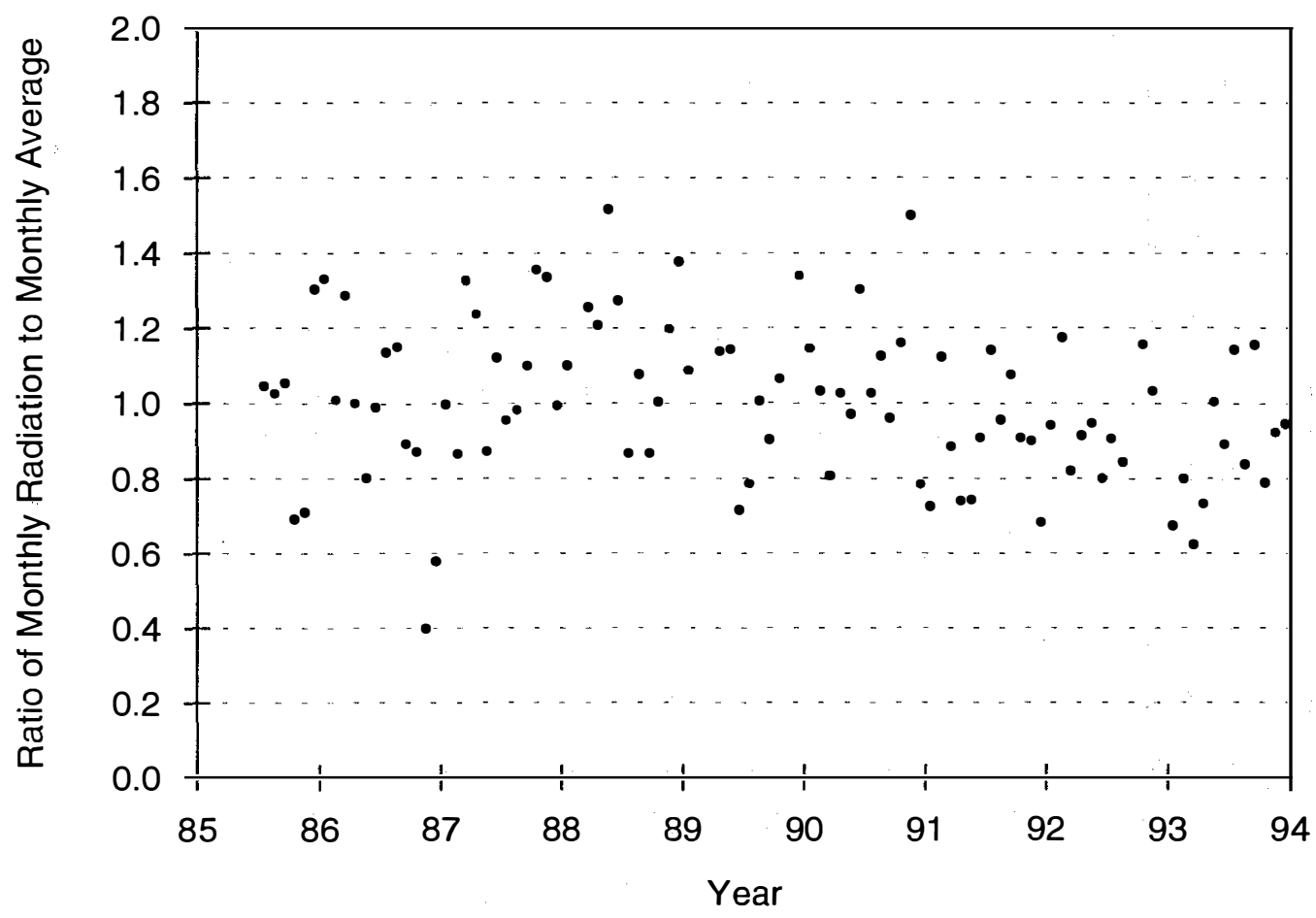

Figure 5-8. Monthly variability of direct normal radiation for Mississippi Valley State University 


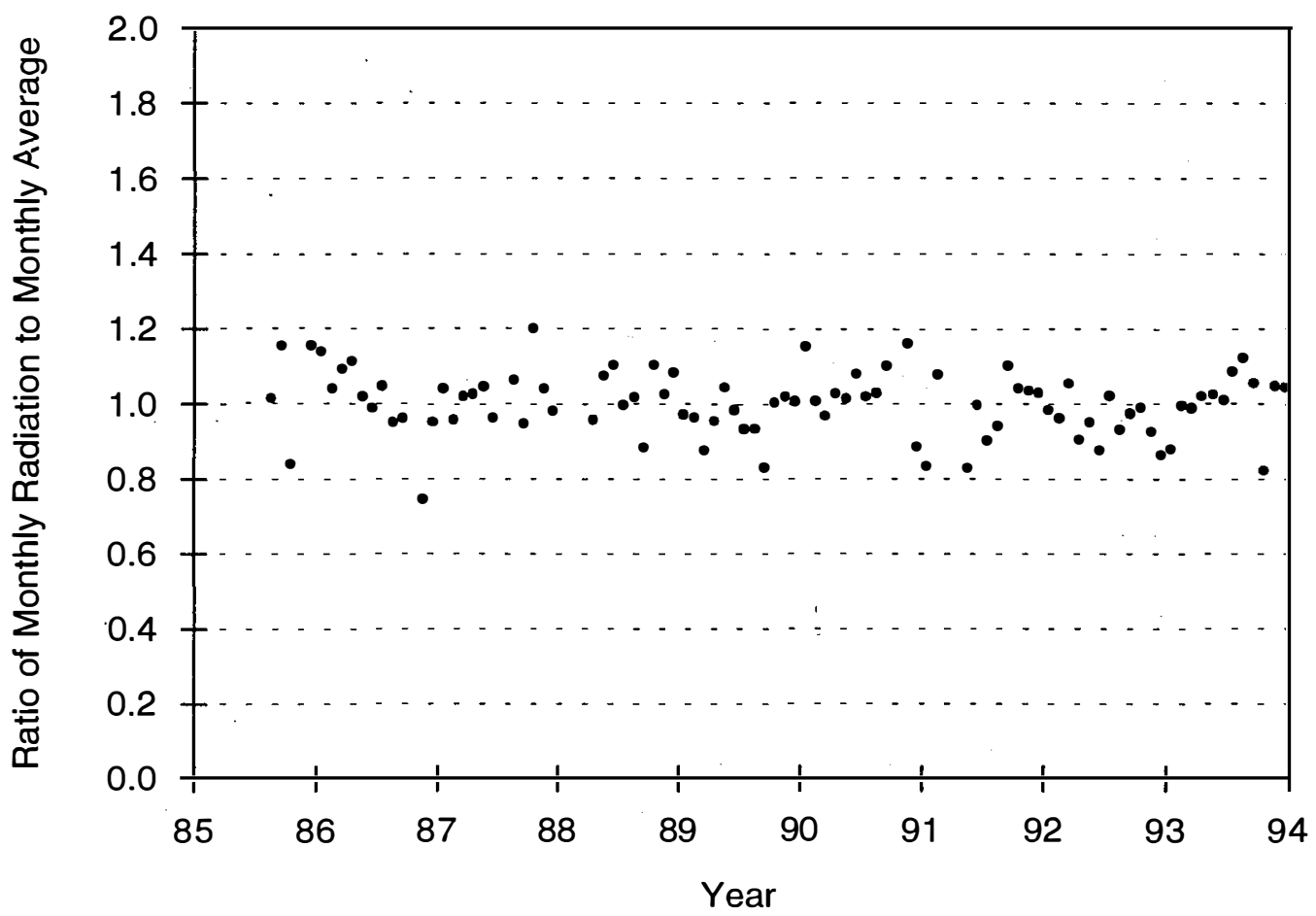

Figure 5-9. Monthly variability of global horizontal radiation for South Carolina State University

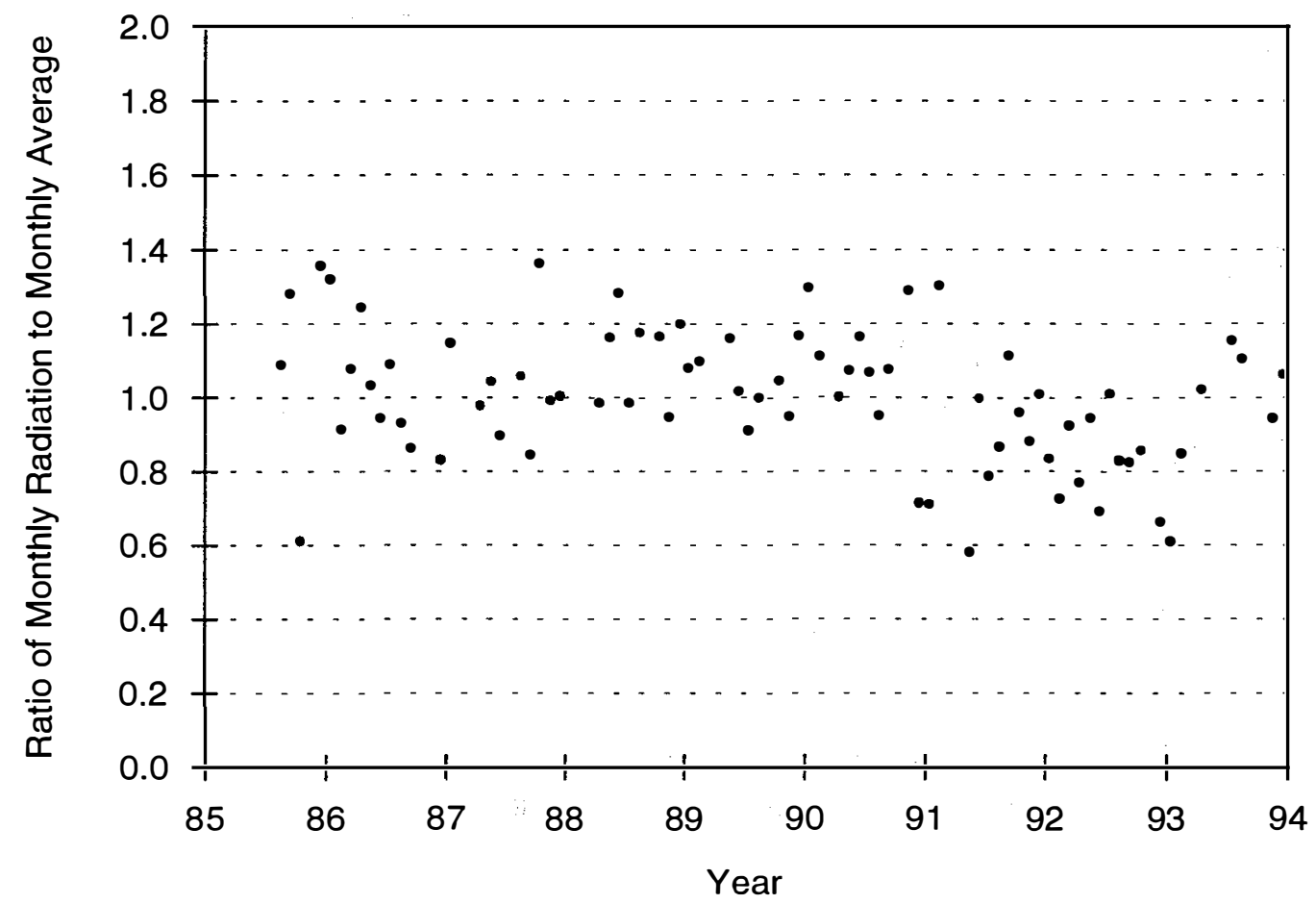

Figure 5-10. Monthly variability of direct normal radiation for South Carolina State University 


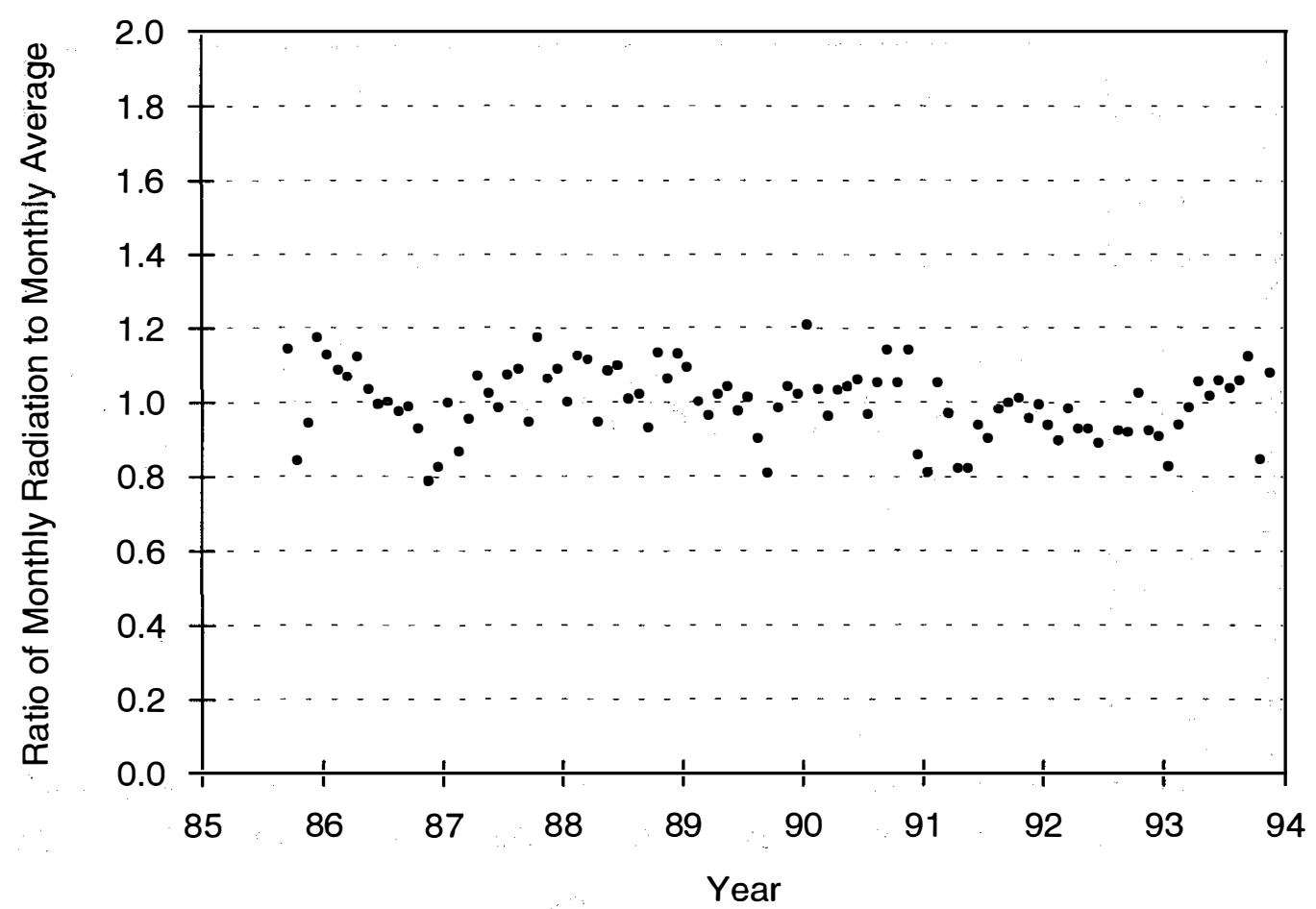

Figure 5-11. Monthly variability of global horizontal radiation for Savannah State College

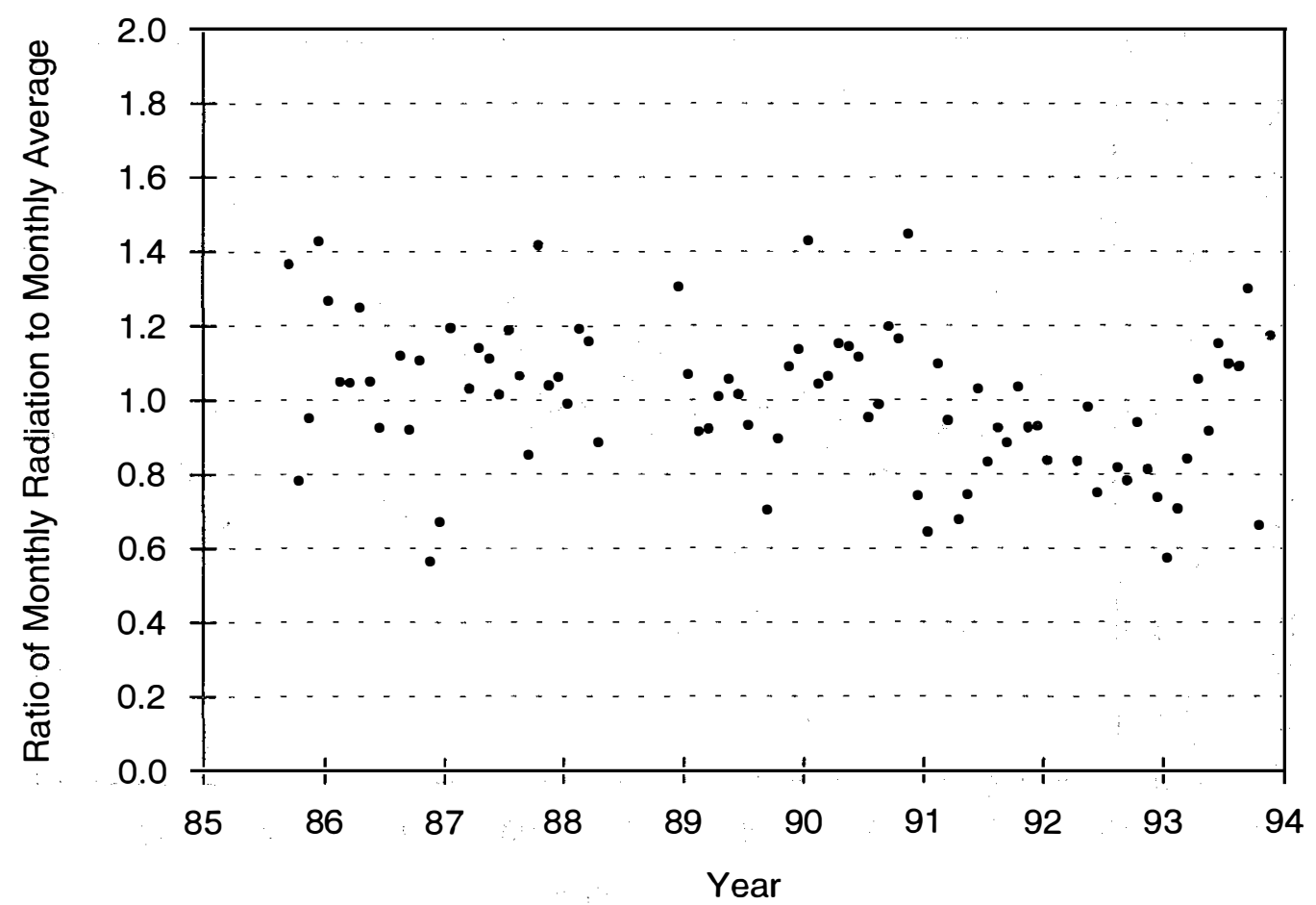

Figure 5-12. Monthly variability of direct normal radiation for Savannah State College 


\subsection{References}

Abernethy, R.; Ringhiser, B. (1985). The History and Statistical Development of the New ASME-SAEAIAA-ISO Measurement and Uncertainty Methodology. 20th AIAA/SAE/ASEE Joint Propulsion Conference; July 1985. AIAA-85-1403. New York: American Institute of Astronautics and Aeronautics.

ASTM. (1986). Standard Method for Calibration of Pyranometers Using the Sum of the Direct and Diffuse Components of Solar Irradiance. Standard E913-R2, Committee E-44, American Society for Testing and Materials.

Holman, J.P. (1971). Experimental Methods for Engineers. 2nd edition. New York: McGraw-Hill.

Hulstrom, R.; Maxwell, G.; Riordan, C.; Stoffel, T. (1988). SERI Resource Assessment Project: FY 1987 Annual Progress Report. SERI/TP-215-338. Golden, CO: Solar Energy Research Institute.

Iqbal, M. (1983). An Introduction to Solar Radiation. New York: Academic Press, Inc.

Marion, W. (1993). Summary Information and Data Sets for NREL's Solar Radiation Research Laboratory, 1981-1991. NREL/TP-463-5217. Golden, CO: National Renewable Energy Laboratory.

Michalsky, J.; Perez, R.; Seals, R.; Ineichen, P. (1994). "Degradation of Solar Concentrator Performance in the Aftermath of Mount Pinatubo." Solar Energy 52(2); pp. 205-213.

NREL. (1993). User's Manual for SERI QC Software-Assessing the Quality of Solar Radiation Data. NREL/TP-463-5608. Golden, CO: National Renewable Energy Laboratory.

Stoffel, T.; Maxwell, E.; Bird, R.; Myers, D. (1987). "Solar Irradiance on Vertical Surfaces." Passive Solar Journal 4(2); pp. 187-210. 


\section{Appendix}

Instrument and Quality Assessment Summaries 


\section{Appendix Table of Contents}

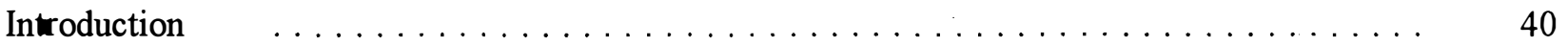

A.1 Global Hórizontal Radiation for Bethune-Cookman College . . . . . . . . . . . . 41

A.2 Diffuse Horizontal Radiation for Bethune-Cookman College . . . . . . . . . . . . 42

A.3 Direct Normal Radiation from Calculations for Bethune-Cookman College . . . . . . 43

A.4 Global Horizontal Radiation for Bluefield State College . . . . . . . . . . . . . . . . . 44

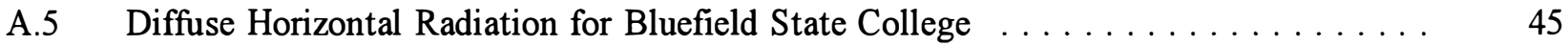

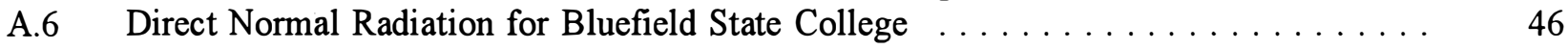

$\begin{aligned} \text { A.7 } & \text { Direct Normal Radiation from Measurements and/or Calculations for } \\ & \text { Bluefield State College } \ldots \ldots \ldots \ldots \ldots \ldots \ldots \ldots \ldots \ldots \ldots \ldots \ldots \ldots\end{aligned}$

A.8 Global Horizontal Radiation for Elizabeth City State University . . . . . . . . . . . . . . 48

A.9 Diffuse Horizontal Radiation for Elizabeth City State University . . . . . . . . . . . . 49

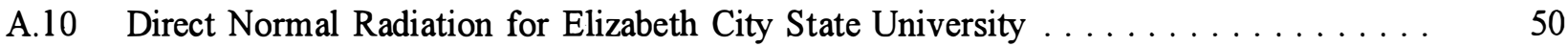

A.11 Direct Normal Radiation from Measurements and/or Calculations for

Elizabeth City State University . . . . . . . . . . . . . . . . . . . . . . . . 51

A.12 Global Horizontal Radiation for Mississippi Valley State University . . . . . . . . 52

A.13 Diffuse Horizontal Radiation for Mississippi Valley State University . . . . . . . . . . 53

A.14 Direct Normal Radiation for Mississippi Valley State University . . . . . . . . . . . . 54

A.15 Direct Normal Radiation from Measurements and/or Calculations for
Mississippi Valley State University $\ldots \ldots \ldots \ldots \ldots \ldots \ldots \ldots \ldots \ldots \ldots$

A.16 Global Horizontal Radiation for South Carolina State University . . . . . . . . . . . 56

A.17 Diffuse Horizontal Radiation for South Carolina State University . . . . . . . . . . . 57

A.18 Direct Normal Radiation from Calculations for South Carolina State University . . . . 58

A.19 Global Horizontal Radiation for Savannah State College . . . . . . . . . . . . . . . . 59

A.20 Diffuse Horizontal Radiation for Savannah State College . . . . . . . . . . . . . . . 60

A.21 Direct Normal Radiation from Calculations for Savannah State College . . . . . . . . . 61 


\section{Introduction}

This appendix contains tables showing when instruments and calibration factors were changed. It also provides quality assessment summaries for daylight hours for each month of the period of record.

All HBCU stations had two pyranometers, one for measuring global horizontal radiation, and one used with a shadowband for measuring diffuse horizontal radiation. For measuring direct normal radiation, three stations are equipped with pyrheliometers: Bluefield State College, Elizabeth City State University, and Mississippi Valley State University. Bluefield State College made direct normal measurements for the complete period of record. The other two stations made direct normal measurements for only the later years indicated in Tables A-20 and A-28.

The quality assessment summary tables address measured values and calculated values. For measured values of global horizontal radiation, diffuse horizontal radiation, and direct normal radiation the tables give two numbers for each month: (1) the percentage of possible data collected and (2) the percentage of data that passes their quality assessment (shown as boldfaced numbers in the tables). The percentages are based on the total daylight hours possible for each month.

For stations that did not make direct normal measurements, or if the data are missing, direct normal radiation may be calculated using global horizontal radiation and diffuse horizontal radiation measurements, if these two measurements pass their quality assessment. Two tables, with different quality assessment criteria, give percentages of total daylight hours for which either the measured direct normal radiation passes its quality assessment or the measured global horizontal radiation and the diffuse horizontal radiation pass their quality assessment and can be used to calculate the direct normal radiation. The first table uses SERI QC as the quality assessment test. The second table also uses SERI QC, but performs an additional test to check diffuse horizontal radiation data for proper alignment of the shadowband. For cloudy skies, diffuse horizontal radiation data may pass SERI QC even though the shadowband is misaligned. Section 5.1 also discusses how these tables were derived. 


\section{A.1 Global Horizontal Radiation for Bethune-Cookman College}

Sensor Instrument: Eppley Laboratory pyranometer, model PSP

Table A-1. Record of Instrument Changes and Calibration Factors

\begin{tabular}{|c|c|c|}
\hline Date & Serial Number & $\begin{array}{c}\text { Calibration Factor } \\
\left(\mu \mathrm{V} / \mathrm{W} / \mathrm{m}^{2}\right)\end{array}$ \\
\hline $8 / 2 / 85$ & $25820 \mathrm{~F} 3$ & 9.850 \\
$2 / 3 / 87$ & $17897 \mathrm{~F} 3$ & 7.843 \\
$12 / 8 / 89$ & $17800 \mathrm{~F} 3$ & 8.881 \\
$4 / 11 / 91$ & $13434 \mathrm{~F} 3$ & 9.107 \\
$6 / 11 / 92$ & $17800 \mathrm{~F} 3$ & 8.805 \\
\hline
\end{tabular}

Table A-2. Percent of Possible Daytime Data Collected and Percent Possibly Passing SERI QC

\begin{tabular}{|r|rrrrrrrrrrrrr|}
\hline Year & Jan & Feb & Mar & Apr & May & June & July & Aug & Sept & Oct & Nov & Déc \\
\hline \multirow{2}{*}{85} & & & & & & & & 85 & 91 & 100 & 93 & 100 \\
& & & & & & & & $\mathbf{8 1}$ & $\mathbf{8 8}$ & $\mathbf{9 7}$ & $\mathbf{9 3}$ & $\mathbf{9 9}$ \\
86 & 100 & 87 & 90 & 100 & 94 & 100 & 37 & 59 & 99 & 76 & 2 & 98 \\
& $\mathbf{9 1}$ & $\mathbf{8 0}$ & $\mathbf{8 9}$ & $\mathbf{9 8}$ & $\mathbf{6 8}$ & $\mathbf{8 0}$ & $\mathbf{2 8}$ & $\mathbf{5 4}$ & $\mathbf{7 9}$ & $\mathbf{4 5}$ & $\mathbf{1}$ & $\mathbf{4 9}$ \\
87 & 6 & 43 & 27 & 25 & 30 & 0 & 95 & 21 & 7 & 100 & 100 & 100 \\
& $\mathbf{6}$ & $\mathbf{3 7}$ & $\mathbf{0}$ & $\mathbf{2}$ & $\mathbf{1 6}$ & $\mathbf{0}$ & $\mathbf{9 4}$ & $\mathbf{2 0}$ & $\mathbf{7}$ & $\mathbf{8 5}$ & $\mathbf{5 9}$ & $\mathbf{3 9}$ \\
88 & 100 & 100 & 100 & 100 & 100 & 100 & 100 & 100 & 100 & 100 & 100 & 98 \\
& $\mathbf{4 6}$ & $\mathbf{4 9}$ & $\mathbf{6 1}$ & $\mathbf{9 4}$ & $\mathbf{9 4}$ & $\mathbf{9 9}$ & $\mathbf{9 0}$ & $\mathbf{9 9}$ & $\mathbf{9 4}$ & $\mathbf{9 0}$ & $\mathbf{6 7}$ & $\mathbf{6 4}$ \\
89 & 100 & 100 & 100 & 100 & 98 & 100 & 100 & 100 & 100 & 100 & 100 & 92 \\
& $\mathbf{9 4}$ & $\mathbf{9 7}$ & $\mathbf{8 9}$ & $\mathbf{9 2}$ & $\mathbf{9 8}$ & $\mathbf{9 7}$ & $\mathbf{9 9}$ & $\mathbf{9 7}$ & $\mathbf{9 1}$ & $\mathbf{9 5}$ & $\mathbf{9 8}$ & $\mathbf{8 5}$ \\
90 & 100 & 89 & 99 & 97 & 98 & 100 & 96 & 96 & 100 & 100 & 100 & 98 \\
& $\mathbf{9 9}$ & $\mathbf{8 5}$ & $\mathbf{9 7}$ & $\mathbf{9 6}$ & $\mathbf{9 7}$ & $\mathbf{9 8}$ & $\mathbf{9 3}$ & $\mathbf{9 2}$ & $\mathbf{9 8}$ & $\mathbf{9 8}$ & $\mathbf{9 7}$ & $\mathbf{7 9}$ \\
91 & 89 & 98 & 99 & 100 & 100 & 97 & 98 & 100 & 100 & 100 & 100 & 100 \\
& $\mathbf{6}$ & $\mathbf{3 5}$ & $\mathbf{6 4}$ & $\mathbf{8 7}$ & $\mathbf{9 2}$ & $\mathbf{9 5}$ & $\mathbf{9 1}$ & $\mathbf{9 5}$ & $\mathbf{9 8}$ & $\mathbf{9 4}$ & $\mathbf{9 7}$ & $\mathbf{9 9}$ \\
92 & 39 & 99 & 100 & 100 & 100 & 100 & 0 & 44 & 97 & 99 & 100 & 94 \\
& $\mathbf{9 6}$ & $\mathbf{9 1}$ & $\mathbf{9 5}$ & $\mathbf{9 4}$ & $\mathbf{9 3}$ & $\mathbf{9 4}$ & $\mathbf{0}$ & $\mathbf{4 1}$ & $\mathbf{9 0}$ & $\mathbf{8 8}$ & $\mathbf{9 3}$ & $\mathbf{9 3}$ \\
93 & 100 & 99 & 98 & 98 & 100 & 70 & 97 & 100 & 100 & 97 & 100 & 100 \\
& $\mathbf{8 9}$ & $\mathbf{9 0}$ & $\mathbf{9 2}$ & $\mathbf{9 2}$ & $\mathbf{7 4}$ & $\mathbf{5 4}$ & $\mathbf{7 3}$ & $\mathbf{8 3}$ & $\mathbf{7 6}$ & $\mathbf{7 3}$ & $\mathbf{8 1}$ & $\mathbf{7 7}$ \\
& & & & & & & & & & & & \\
& & & & & & & & & & &
\end{tabular}

Note: Top number is percentage of possible data collected; boldfaced number is percentage of data passing SERI QC. 


\section{A.2 Diffuse Horizontal Radiation for Bethune-Cookman College}

Sensor Instrument: Eppley Laboratory pyranometer, model PSP with a shadowband

Table A-3. Record of Instrument Changes and Calibration Factors

\begin{tabular}{|c|c|c|}
\hline Date & Serial Number & $\begin{array}{c}\text { Calibration Factor } \\
\left(\mu \mathrm{V} / \mathrm{W} / \mathrm{m}^{2}\right)\end{array}$ \\
\hline $8 / 2 / 85$ & $25782 \mathrm{~F} 3$ & 10.179 \\
$2 / 3 / 87$ & $25824 \mathrm{~F} 3$ & 10.132 \\
$12 / 8 / 89$ & $17879 \mathrm{~F} 3$ & 8.617 \\
$4 / 11 / 91$ & $25951 \mathrm{~F} 3$ & 8.091 \\
$6 / 11 / 92$ & $17879 \mathrm{~F} 3$ & 8.520 \\
\hline
\end{tabular}

Table A-4. Percent of Possible Daytime Data Collected and Percent Possibly Passing SERI QC

\begin{tabular}{|r|rrrrrrrrrrrr|}
\hline Year & Jan & Feb & Mar & \multicolumn{1}{c}{ Apr } & May & June & July & Aug & Sept & Oct & Nov & Dec \\
\hline 85 & & & & & & & & 85 & 91 & 100 & 93 & 100 \\
& & & & & & & & $\mathbf{8 3}$ & $\mathbf{8 9}$ & $\mathbf{9 8}$ & $\mathbf{9 3}$ & $\mathbf{9 9}$ \\
86 & 100 & 87 & 90 & 100 & 94 & 100 & 37 & 59 & 99 & 76 & 2 & 98 \\
& $\mathbf{9 4}$ & $\mathbf{8 1}$ & $\mathbf{8 9}$ & $\mathbf{9 8}$ & $\mathbf{7 8}$ & $\mathbf{8 8}$ & $\mathbf{3 1}$ & $\mathbf{5 2}$ & $\mathbf{8 6}$ & $\mathbf{6 5}$ & $\mathbf{2}$ & $\mathbf{9 6}$ \\
87 & 6 & 43 & 27 & 24 & 30 & 0 & 95 & 21 & 7 & 100 & 100 & 100 \\
& $\mathbf{6}$ & $\mathbf{3 9}$ & $\mathbf{2 7}$ & $\mathbf{2 2}$ & $\mathbf{2 8}$ & $\mathbf{0}$ & $\mathbf{9 4}$ & $\mathbf{2 1}$ & $\mathbf{7}$ & $\mathbf{9 3}$ & $\mathbf{9 7}$ & $\mathbf{9 5}$ \\
88 & 100 & 100 & 100 & 100 & 100 & 100 & 100 & 100 & 100 & 100 & 100 & 98 \\
& $\mathbf{9 4}$ & $\mathbf{9 1}$ & $\mathbf{9 1}$ & $\mathbf{9 7}$ & $\mathbf{9 8}$ & $\mathbf{9 9}$ & $\mathbf{9 7}$ & $\mathbf{9 6}$ & $\mathbf{8 0}$ & $\mathbf{9 7}$ & $\mathbf{8 6}$ & $\mathbf{9 4}$ \\
89 & 100 & 100 & 100 & 100 & 98 & 100 & 100 & 100 & 100 & 100 & 100 & 92 \\
& $\mathbf{9 5}$ & $\mathbf{9 8}$ & $\mathbf{9 1}$ & $\mathbf{8 5}$ & $\mathbf{9 8}$ & $\mathbf{9 9}$ & $\mathbf{9 9}$ & $\mathbf{9 3}$ & $\mathbf{9 6}$ & $\mathbf{9 6}$ & $\mathbf{9 9}$ & $\mathbf{8 7}$ \\
90 & 100 & 89 & 99 & 97 & 98 & 100 & 96 & 96 & 100 & 100 & 100 & 98 \\
& $\mathbf{9 9}$ & $\mathbf{8 6}$ & $\mathbf{9 7}$ & $\mathbf{9 6}$ & $\mathbf{9 7}$ & $\mathbf{9 9}$ & $\mathbf{9 5}$ & $\mathbf{9 3}$ & $\mathbf{9 9}$ & $\mathbf{9 7}$ & $\mathbf{9 3}$ & $\mathbf{9 6}$ \\
91 & 89 & 98 & 99 & 100 & 100 & 97 & 98 & 100 & 100 & 100 & 100 & 100 \\
& $\mathbf{8 2}$ & $\mathbf{4 4}$ & $\mathbf{9}$ & $\mathbf{6 1}$ & $\mathbf{6 6}$ & $\mathbf{9 5}$ & $\mathbf{7 9}$ & $\mathbf{5 1}$ & $\mathbf{7 8}$ & $\mathbf{4 6}$ & $\mathbf{5 6}$ & $\mathbf{6 3}$ \\
92 & 39 & 99 & 100 & 100 & 100 & $\mathbf{1 0}$ & 0 & 44 & 97 & 99 & 100 & 94 \\
& $\mathbf{1 8}$ & $\mathbf{5 9}$ & $\mathbf{6 1}$ & $\mathbf{5 3}$ & $\mathbf{3 1}$ & $\mathbf{8 0}$ & $\mathbf{0}$ & $\mathbf{1 7}$ & $\mathbf{4 8}$ & $\mathbf{4 2}$ & $\mathbf{6 1}$ & $\mathbf{5 6}$ \\
93 & 100 & 99 & 98 & 98 & 100 & $\mathbf{7 0}$ & 97 & 100 & 100 & 97 & 100 & 100 \\
& $\mathbf{5 7}$ & $\mathbf{4 5}$ & $\mathbf{4 8}$ & $\mathbf{3 1}$ & $\mathbf{8 3}$ & $\mathbf{6 0}$ & $\mathbf{8 0}$ & $\mathbf{7 6}$ & $\mathbf{3 4}$ & $\mathbf{4 8}$ & $\mathbf{5 5}$ & $\mathbf{4 3}$ \\
& & & & & & & & & & & & \\
\hline
\end{tabular}

Note: Top number is percentage of possible data collected; boldfaced number is percentage of data passing SERI QC. 


\section{A.3 Direct Normal Radiation from Calculations for Bethune-Cookman College}

Table A-5. Percent of Possible Daytime Data Passing SERI QC

\begin{tabular}{|r|rrrrrrrrrrrrr|}
\hline Year & Jan & Feb & Mar & Apr & May & June & July & Aug & Sept & Oct & Nov & Dec \\
\hline 85 & & & & & & & & 81 & 88 & 97 & 93 & 99 \\
86 & 91 & 80 & 89 & 98 & 68 & 78 & 28 & 51 & 77 & 45 & 1 & 49 \\
87 & 6 & 37 & 0 & 2 & 16 & 0 & 93 & 20 & 7 & 82 & 59 & 39 \\
88 & 46 & 49 & 61 & 94 & 94 & 99 & 90 & 95 & 76 & 90 & 58 & 64 \\
89 & 93 & 97 & 84 & 80 & 98 & 97 & 99 & 92 & 89 & 95 & 98 & 85 \\
90 & 99 & 85 & 97 & 96 & 97 & 98 & 93 & 92 & 98 & 97 & 92 & 79 \\
91 & 6 & 3 & 2 & 60 & 65 & 95 & 78 & 50 & 78 & 45 & 55 & 62 \\
92 & 18 & 59 & 61 & 53 & 31 & 79 & 0 & 17 & 47 & 40 & 61 & 56 \\
93 & 57 & 44 & 47 & 30 & 73 & 52 & 72 & 71 & 33 & 45 & 54 & 43 \\
& & & & & & & & & & & & \\
\hline
\end{tabular}

Table A-6. Percent of Possible Daytime Data Passing SERI QC and Shadowband Alignment Tests

\begin{tabular}{|r|rrrrrrrrrrrrr|}
\hline Year & Jan & Feb & \multicolumn{1}{c}{ Mar } & \multicolumn{1}{c}{ Apr } & May & June & July & Aug & Sept & Oct & Nov & Dec \\
\hline 85 & & & & & & & & 80 & 84 & 97 & 90 & 98 \\
86 & 88 & 80 & 89 & 98 & 54 & 66 & 21 & 44 & 65 & 42 & 1 & 49 \\
87 & 6 & 33 & 0 & 1 & 16 & 0 & 93 & 20 & 7 & 69 & 56 & 39 \\
88 & 41 & 49 & 61 & 94 & 94 & 99 & 89 & 92 & 58 & 90 & 49 & 64 \\
89 & 89 & 91 & 82 & 74 & 98 & 97 & 99 & 89 & 84 & 92 & 98 & 85 \\
90 & 98 & 78 & 96 & 96 & 97 & 98 & 93 & 92 & 98 & 97 & 84 & 79 \\
91 & 5 & 3 & 2 & 60 & 37 & 94 & 59 & 21 & 60 & 0 & 0 & 0 \\
92 & 0 & 0 & 27 & 25 & 0 & 64 & 0 & 1 & 0 & 0 & 0 & 0 \\
93 & 0 & 0 & 0 & 1 & 50 & 35 & 56 & 43 & 0 & 0 & 0 & 4 \\
& & & & & & & & & & & & \\
\hline
\end{tabular}




\section{A.4 Global Horizontal Radiation for Bluefield State College}

Sensor Instrument: Eppley Laboratory pyranometer, model PSP

Table A-7. Record of Instrument Changes and Calibration Factors

\begin{tabular}{|c|c|c|}
\hline Date & Serial Number & $\begin{array}{c}\text { Calibration Factor } \\
\left(\mu \mathrm{V} / \mathrm{W} / \mathrm{m}^{2}\right)\end{array}$ \\
\hline $11 / 6 / 85$ & $25821 \mathrm{~F} 3$ & 9.740 \\
$12 / 3 / 87$ & $25819 \mathrm{~F} 3$ & 9.550 \\
$9 / 19 / 89$ & $17818 \mathrm{~F} 3$ & 7.975 \\
$6 / 2 / 92$ & $25821 \mathrm{~F} 3$ & 9.420 \\
\hline
\end{tabular}

Table A-8. Percent of Possible Daytime Data Collected and Percent Possibly Passing SERI QC

\begin{tabular}{|r|rrrrrrrrrrrr|}
\hline Year & Jan & Feb & Mar & Apr & May & June & July & Aug & Sept & Oct & Nov & Dec \\
\hline 85 & & & & & & & & & & & 79 & 94 \\
& & & & & & & & & & & $\mathbf{7 3}$ & $\mathbf{8 4}$ \\
86 & 99 & 96 & 0 & 0 & 95 & 100 & 100 & 100 & 100 & 96 & 98 & 100 \\
& $\mathbf{9 5}$ & $\mathbf{8 9}$ & $\mathbf{0}$ & $\mathbf{0}$ & $\mathbf{9 3}$ & $\mathbf{9 8}$ & $\mathbf{9 7}$ & $\mathbf{9 8}$ & $\mathbf{9 6}$ & $\mathbf{8 8}$ & $\mathbf{9 2}$ & $\mathbf{9 0}$ \\
87 & 100 & 93 & 100 & 35 & 97 & 100 & 39 & 88 & 100 & 98 & 99 & 97 \\
& $\mathbf{9 3}$ & $\mathbf{8 9}$ & $\mathbf{9 7}$ & $\mathbf{3 2}$ & $\mathbf{9 5}$ & $\mathbf{9 6}$ & $\mathbf{3 7}$ & $\mathbf{8 7}$ & $\mathbf{9 7}$ & $\mathbf{9 2}$ & $\mathbf{8 9}$ & $\mathbf{9 3}$ \\
88 & 100 & 100 & 100 & 100 & 8 & 99 & 100 & 100 & 100 & 100 & 100 & 100 \\
& $\mathbf{9 4}$ & $\mathbf{9 2}$ & $\mathbf{9 8}$ & $\mathbf{9 5}$ & $\mathbf{7}$ & $\mathbf{9 4}$ & $\mathbf{9 8}$ & $\mathbf{9 8}$ & $\mathbf{9 7}$ & $\mathbf{9 5}$ & $\mathbf{9 5}$ & $\mathbf{9 3}$ \\
89 & 100 & 100 & 100 & 100 & 100 & 100 & 100 & 100 & 87 & 100 & 100 & 100 \\
& $\mathbf{9 7}$ & $\mathbf{9 3}$ & $\mathbf{9 4}$ & $\mathbf{9 5}$ & $\mathbf{9 4}$ & $\mathbf{9 9}$ & $\mathbf{9 7}$ & $\mathbf{9 8}$ & $\mathbf{8 5}$ & $\mathbf{8 5}$ & $\mathbf{8 4}$ & $\mathbf{8 5}$ \\
90 & 100 & 100 & 100 & 99 & 100 & 100 & 100 & 100 & 100 & 100 & 100 & 100 \\
& $\mathbf{9 6}$ & $\mathbf{9 6}$ & $\mathbf{9 0}$ & $\mathbf{9 1}$ & $\mathbf{9 7}$ & $\mathbf{9 7}$ & $\mathbf{9 8}$ & $\mathbf{9 9}$ & $\mathbf{9 7}$ & $\mathbf{9 2}$ & $\mathbf{9 7}$ & $\mathbf{9 6}$ \\
91 & 100 & 100 & 100 & 100 & 100 & 100 & 100 & 100 & 100 & 100 & 100 & 100 \\
& $\mathbf{9 5}$ & $\mathbf{9 7}$ & $\mathbf{9 3}$ & $\mathbf{9 7}$ & $\mathbf{9 8}$ & $\mathbf{9 7}$ & $\mathbf{9 9}$ & $\mathbf{9 6}$ & $\mathbf{9 7}$ & $\mathbf{9 8}$ & $\mathbf{9 5}$ & $\mathbf{9 3}$ \\
92 & 100 & 100 & 100 & 100 & 100 & 30 & 93 & 99 & 47 & 96 & 100 & 86 \\
& $\mathbf{9 9}$ & $\mathbf{9 8}$ & $\mathbf{9 5}$ & $\mathbf{9 6}$ & $\mathbf{9 6}$ & $\mathbf{3 0}$ & $\mathbf{9 0}$ & $\mathbf{9 6}$ & $\mathbf{4 3}$ & $\mathbf{9 4}$ & $\mathbf{9 7}$ & $\mathbf{8 2}$ \\
93 & 97 & 96 & 98 & 93 & 89 & 90 & 100 & 100 & 77 & 77 & 100 & 100 \\
& $\mathbf{9 5}$ & $\mathbf{9 5}$ & $\mathbf{9 3}$ & $\mathbf{8 9}$ & $\mathbf{8 7}$ & $\mathbf{7 8}$ & $\mathbf{9 8}$ & $\mathbf{9 1}$ & $\mathbf{6 8}$ & $\mathbf{7 3}$ & $\mathbf{8 3}$ & $\mathbf{8 9}$ \\
& & & & & & & & & & & & \\
& & & & & & & & & & &
\end{tabular}

Note: Top number is percentage of possible data collected; boldfaced number is percentage of data passing SERI QC. 


\section{A.5 Diffuse Horizontal Radiation for Bluefield State College}

Sensor Instrument: Eppley Laboratory pyranometer, model PSP with a shadowband

Table A-9. Record of Instrument Changes and Calibration Factors

\begin{tabular}{|c|c|c|}
\hline Date & Serial Number & $\begin{array}{c}\text { Calibration Factor } \\
\left(\mu \mathrm{V} / \mathrm{W} / \mathrm{m}^{2}\right)\end{array}$ \\
\hline $11 / 6 / 85$ & $25822 \mathrm{~F} 3$ & 9.910 \\
$12 / 3 / 87$ & $25823 \mathrm{~F} 3$ & 9.823 \\
$9 / 19 / 89$ & $17860 \mathrm{~F} 3$ & 7.740 \\
$6 / 2 / 92$ & $17880 \mathrm{~F} 3$ & 8.691 \\
\hline
\end{tabular}

Table A-10. Percent of Possible Daytime Data Collected and Percent Possibly Passing SERI QC

\begin{tabular}{|r|rrrrrrrrrrrr|}
\hline Year & Jan & Feb & Mar & Apr & May & June & July & Aug & Sept & Oct & Nov & Dec \\
\hline \multirow{2}{*}{85} & & & & & & & & & & & 79 & 94 \\
& & & & & & & & & & & $\mathbf{7 4}$ & $\mathbf{8 5}$ \\
86 & 99 & 96 & 0 & 0 & 95 & 100 & 100 & 100 & 100 & 96 & 98 & 100 \\
& $\mathbf{9 7}$ & $\mathbf{9 0}$ & $\mathbf{0}$ & $\mathbf{0}$ & $\mathbf{9 4}$ & $\mathbf{9 8}$ & $\mathbf{9 8}$ & $\mathbf{9 8}$ & $\mathbf{9 7}$ & $\mathbf{8 9}$ & $\mathbf{9 4}$ & $\mathbf{9 1}$ \\
87 & 100 & 93 & 100 & 35 & 97 & 100 & 39 & 88 & 100 & 98 & 99 & 97 \\
& $\mathbf{9 6}$ & $\mathbf{9 0}$ & $\mathbf{9 8}$ & $\mathbf{3 2}$ & $\mathbf{9 5}$ & $\mathbf{9 6}$ & $\mathbf{3 7}$ & $\mathbf{8 7}$ & $\mathbf{9 7}$ & $\mathbf{9 4}$ & $\mathbf{9 0}$ & $\mathbf{9 5}$ \\
88 & 100 & 100 & 100 & 100 & 8 & 99 & 100 & 100 & 100 & 100 & 100 & 100 \\
& $\mathbf{9 6}$ & $\mathbf{9 3}$ & $\mathbf{9 9}$ & $\mathbf{9 6}$ & $\mathbf{7}$ & $\mathbf{9 5}$ & $\mathbf{9 8}$ & $\mathbf{9 8}$ & $\mathbf{9 8}$ & $\mathbf{9 7}$ & $\mathbf{9 7}$ & $\mathbf{9 5}$ \\
89 & 100 & 100 & 100 & 100 & 100 & 100 & 100 & 100 & 87 & 100 & 100 & 100 \\
& $\mathbf{9 9}$ & $\mathbf{9 5}$ & $\mathbf{9 6}$ & $\mathbf{9 7}$ & $\mathbf{9 7}$ & $\mathbf{9 9}$ & $\mathbf{9 8}$ & $\mathbf{9 8}$ & $\mathbf{8 6}$ & $\mathbf{8 6}$ & $\mathbf{8 8}$ & $\mathbf{8 5}$ \\
90 & 100 & 100 & 100 & 99 & 100 & 100 & 100 & 100 & 100 & 100 & 100 & 100 \\
& $\mathbf{9 8}$ & $\mathbf{9 7}$ & $\mathbf{9 1}$ & $\mathbf{9 1}$ & $\mathbf{9 8}$ & $\mathbf{9 8}$ & $\mathbf{9 9}$ & $\mathbf{9 9}$ & $\mathbf{9 8}$ & $\mathbf{9 5}$ & $\mathbf{9 8}$ & $\mathbf{9 8}$ \\
91 & 100 & 100 & 100 & 100 & 100 & 100 & 100 & 100 & 100 & 100 & 100 & 100 \\
& $\mathbf{9 7}$ & $\mathbf{9 8}$ & $\mathbf{9 6}$ & $\mathbf{9 8}$ & $\mathbf{9 8}$ & $\mathbf{9 8}$ & $\mathbf{1 0 0}$ & $\mathbf{9 6}$ & $\mathbf{9 8}$ & $\mathbf{9 8}$ & $\mathbf{9 6}$ & $\mathbf{9 5}$ \\
92 & 100 & 100 & 100 & 100 & 100 & 30 & 93 & 99 & 47 & 96 & 100 & 86 \\
& $\mathbf{9 9}$ & $\mathbf{9 9}$ & $\mathbf{9 7}$ & $\mathbf{9 7}$ & $\mathbf{9 8}$ & $\mathbf{3 0}$ & $\mathbf{9 1}$ & $\mathbf{9 7}$ & $\mathbf{4 3}$ & $\mathbf{9 5}$ & $\mathbf{9 7}$ & $\mathbf{8 4}$ \\
93 & 97 & 96 & 98 & 93 & 89 & 90 & 100 & 100 & 77 & $\mathbf{7 7}$ & 100 & 100 \\
& $\mathbf{9 6}$ & $\mathbf{9 5}$ & $\mathbf{9 5}$ & $\mathbf{9 1}$ & $\mathbf{8 8}$ & $\mathbf{7 8}$ & $\mathbf{9 9}$ & $\mathbf{9 1}$ & $\mathbf{6 8}$ & $\mathbf{7 2}$ & $\mathbf{8 4}$ & $\mathbf{9 0}$ \\
& & & & & & & & & & & & \\
& & & & & & & & & & &
\end{tabular}

Note: Top number is percentage of possible data collected; boldfaced number is percentage of data passing SERI QC. 


\section{A.6 Direct Normal Radiation for Bluefield State College}

Sensor Instrument: Eppley Laboratory pyrheliometer, model NIP

Table A-11. Record of Instrument Changes and Calibration Factors

\begin{tabular}{|c|c|c|}
\hline Date & Serial Number & $\begin{array}{c}\text { Calibration Factor } \\
\left(\mu \mathrm{V} / \mathrm{W} / \mathrm{m}^{2}\right)\end{array}$ \\
\hline $11 / 6 / 85$ & $19791 \mathrm{E} 6$ & 8.280 \\
$12 / 7 / 87$ & $25792 \mathrm{E} 6$ & 7.638 \\
$9 / 19 / 89$ & $17828 \mathrm{E} 6$ & 6.482 \\
$4 / 27 / 90$ & $12559 \mathrm{E} 6$ & 7.336 \\
$6 / 2 / 92$ & $19791 \mathrm{E} 6$ & 8.512 \\
\hline
\end{tabular}

Table A-12. Percent of Possible Daytime Data Collected and Percent Possibly Passing SERI QC

\begin{tabular}{|c|c|c|c|c|c|c|c|c|c|c|c|c|}
\hline Year & Jan & Feb & Mar & Apr & May & June & July & Aug & Sept & Oct & Nov & Dec \\
\hline 85 & & & & & & & & & & & $\begin{array}{l}79 \\
74\end{array}$ & $\begin{array}{l}94 \\
85\end{array}$ \\
\hline 86 & $\begin{array}{l}99 \\
97\end{array}$ & $\begin{array}{l}96 \\
92\end{array}$ & $\begin{array}{l}0 \\
\mathbf{0}\end{array}$ & $\begin{array}{l}0 \\
\text { 0 }\end{array}$ & $\begin{array}{l}95 \\
94\end{array}$ & $\begin{array}{r}100 \\
97\end{array}$ & $\begin{array}{r}100 \\
99\end{array}$ & $\begin{array}{r}100 \\
95\end{array}$ & $\begin{array}{r}100 \\
96\end{array}$ & $\begin{array}{l}96 \\
89\end{array}$ & $\begin{array}{l}98 \\
92\end{array}$ & $\begin{array}{r}100 \\
91\end{array}$ \\
\hline 87 & $\begin{array}{r}100 \\
95\end{array}$ & $\begin{array}{l}93 \\
91\end{array}$ & $\begin{array}{r}100 \\
97\end{array}$ & $\begin{array}{l}35 \\
33\end{array}$ & $\begin{array}{l}97 \\
96\end{array}$ & $\begin{array}{r}100 \\
97\end{array}$ & $\begin{array}{l}39 \\
38\end{array}$ & $\begin{array}{l}88 \\
87\end{array}$ & $\begin{array}{r}100 \\
97\end{array}$ & $\begin{array}{l}98 \\
93\end{array}$ & $\begin{array}{l}99 \\
90\end{array}$ & $\begin{array}{l}97 \\
95\end{array}$ \\
\hline 88 & $\begin{array}{r}100 \\
96\end{array}$ & $\begin{array}{r}100 \\
93\end{array}$ & $\begin{array}{r}100 \\
99\end{array}$ & $\begin{array}{r}100 \\
97\end{array}$ & $\begin{array}{l}8 \\
7\end{array}$ & $\begin{array}{l}99 \\
95\end{array}$ & $\begin{array}{r}100 \\
97\end{array}$ & $\begin{array}{r}100 \\
98\end{array}$ & $\begin{array}{r}100 \\
97\end{array}$ & $\begin{array}{r}100 \\
96\end{array}$ & $\begin{array}{r}100 \\
96\end{array}$ & $\begin{array}{r}100 \\
94\end{array}$ \\
\hline 89 & $\begin{array}{r}100 \\
99\end{array}$ & $\begin{array}{r}100 \\
94\end{array}$ & $\begin{array}{r}100 \\
96\end{array}$ & $\begin{array}{r}100 \\
98\end{array}$ & $\begin{array}{r}100 \\
97\end{array}$ & $\begin{array}{r}100 \\
99\end{array}$ & $\begin{array}{r}100 \\
98\end{array}$ & $\begin{array}{r}100 \\
98\end{array}$ & $\begin{array}{l}87 \\
83\end{array}$ & $\begin{array}{r}100 \\
76\end{array}$ & $\begin{array}{r}100 \\
81\end{array}$ & $\begin{array}{r}100 \\
85\end{array}$ \\
\hline 90 & $\begin{array}{r}100 \\
92\end{array}$ & $\begin{array}{r}100 \\
90\end{array}$ & $\begin{array}{r}100 \\
85\end{array}$ & $\begin{array}{l}99 \\
86\end{array}$ & $\begin{array}{r}100 \\
99\end{array}$ & $\begin{array}{r}100 \\
98\end{array}$ & $\begin{array}{r}100 \\
99\end{array}$ & $\begin{array}{r}100 \\
99\end{array}$ & $\begin{array}{r}100 \\
97\end{array}$ & $\begin{array}{r}100 \\
95\end{array}$ & $\begin{array}{r}100 \\
97\end{array}$ & $\begin{array}{r}100 \\
97\end{array}$ \\
\hline 91 & $\begin{array}{r}100 \\
98\end{array}$ & $\begin{array}{r}100 \\
98\end{array}$ & $\begin{array}{r}100 \\
97\end{array}$ & $\begin{array}{r}100 \\
98\end{array}$ & $\begin{array}{r}100 \\
98\end{array}$ & $\begin{array}{r}100 \\
98\end{array}$ & $\begin{array}{l}100 \\
\mathbf{1 0 0}\end{array}$ & $\begin{array}{r}100 \\
96\end{array}$ & $\begin{array}{r}100 \\
98\end{array}$ & $\begin{array}{r}100 \\
99\end{array}$ & $\begin{array}{r}100 \\
97\end{array}$ & $\begin{array}{r}100 \\
96\end{array}$ \\
\hline 92 & $\begin{array}{r}100 \\
99\end{array}$ & $\begin{array}{r}100 \\
99\end{array}$ & $\begin{array}{r}100 \\
98\end{array}$ & $\begin{array}{r}100 \\
98\end{array}$ & $\begin{array}{r}100 \\
98\end{array}$ & $\begin{array}{l}28 \\
28\end{array}$ & $\begin{array}{l}93 \\
91\end{array}$ & $\begin{array}{l}99 \\
97\end{array}$ & $\begin{array}{l}47 \\
43\end{array}$ & $\begin{array}{l}96 \\
95\end{array}$ & $\begin{array}{r}100 \\
98\end{array}$ & $\begin{array}{l}86 \\
85\end{array}$ \\
\hline 93 & $\begin{array}{l}97 \\
96\end{array}$ & $\begin{array}{l}96 \\
95\end{array}$ & $\begin{array}{l}98 \\
95\end{array}$ & $\begin{array}{l}93 \\
91\end{array}$ & $\begin{array}{l}89 \\
88\end{array}$ & $\begin{array}{l}69 \\
19\end{array}$ & $\begin{array}{l}8 \\
7\end{array}$ & $\begin{array}{r}100 \\
92\end{array}$ & $\begin{array}{l}77 \\
69\end{array}$ & $\begin{array}{l}77 \\
72\end{array}$ & $\begin{array}{r}100 \\
84\end{array}$ & $\begin{array}{r}100 \\
88\end{array}$ \\
\hline
\end{tabular}

Note: Top number is percentage of possible data collected; boldfaced number is percentage of data passing SERI QC. 


\section{A.7 Direct Normal Radiation from Measurements and/or Calculations for Bluefield State College}

Table A-13. Percent of Possible Daytime Data Passing SERI QC

\begin{tabular}{|c|ccccccccccccc|}
\hline Year & Jan & Feb & Mar & Apr & May & June & July & Aug & Sept & Oct & Nov & Dec \\
\hline 85 & & & & & & & & & & & 74 & 85 \\
86 & 97 & 92 & 0 & 0 & 94 & 98 & 99 & 98 & 96 & 89 & 94 & 92 \\
87 & 95 & 91 & 98 & 33 & 96 & 97 & 38 & 87 & 97 & 94 & 91 & 95 \\
88 & 96 & 94 & 99 & 97 & 7 & 95 & 98 & 99 & 98 & 98 & 97 & 95 \\
89 & 99 & 95 & 96 & 98 & 97 & 99 & 98 & 99 & 86 & 87 & 86 & 88 \\
90 & 97 & 96 & 93 & 92 & 99 & 98 & 99 & 99 & 98 & 95 & 98 & 98 \\
91 & 98 & 98 & 97 & 98 & 98 & 98 & 100 & 96 & 98 & 99 & 97 & 96 \\
92 & 99 & 99 & 98 & 98 & 98 & 30 & 91 & 97 & 43 & 95 & 98 & 85 \\
93 & 96 & 95 & 95 & 91 & 88 & 78 & 98 & 92 & 69 & 73 & 85 & 90 \\
& & & & & & & & & & & & \\
\hline
\end{tabular}

Table A-14. Percent of Possible Daytime Data Passing SERI QC and Shadowband Alignment Tests

\begin{tabular}{|c|rrrrrrrrrrrr|}
\hline Year & Jan & Feb & Mar & Apr & May & June & July & Aug & Sept & Oct & Nov & Dec \\
\hline 85 & & & & & & & & & & & 74 & 85 \\
86 & 97 & 92 & 0 & 0 & 94 & 98 & 99 & 98 & 96 & 89 & 94 & 92 \\
87 & 95 & 91 & 98 & 33 & 96 & 97 & 38 & 87 & 97 & 94 & 91 & 95 \\
88 & 96 & 94 & 99 & 97 & 7 & 95 & 98 & 99 & 98 & 98 & 97 & 95 \\
89 & 99 & 95 & 96 & 98 & 97 & 99 & 98 & 99 & 86 & 87 & 86 & 88 \\
90 & 97 & 96 & 93 & 92 & 99 & 98 & 99 & 99 & 98 & 95 & 98 & 98 \\
91 & 98 & 98 & 97 & 98 & 98 & 98 & 100 & 96 & 98 & 99 & 97 & 96 \\
92 & 99 & 99 & 98 & 98 & 98 & 30 & 91 & 97 & 43 & 95 & 98 & 85 \\
93 & 96 & 95 & 95 & 91 & 88 & 75 & 98 & 92 & 69 & 72 & 84 & 88 \\
& & & & & & & & & & & &. \\
\hline
\end{tabular}




\section{A.8 Global Horizontal Radiation for Elizabeth City State University}

Sensor Instrument: Eppley Laboratory pyranometer, model PSP

Table A-15. Record of Instrument Changes and Calibration Factors

\begin{tabular}{|c|c|c|}
\hline Date & Serial Number & $\begin{array}{c}\text { Calibration Factor } \\
\left(\mu \mathrm{V} / \mathrm{W} / \mathrm{m}^{2}\right)\end{array}$ \\
\hline $9 / 3 / 85$ & $25823 \mathrm{~F} 3$ & 10.393 \\
$10 / 1 / 87$ & $18041 \mathrm{~F} 3$ & 7.295 \\
$5 / 2 / 89$ & $24191 \mathrm{~F} 3$ & 8.931 \\
$8 / 9 / 89$ & $25822 \mathrm{~F} 3$ & 9.812 \\
$2 / 15 / 90$ & $25823 / \mathrm{f} 3$ & 9.707 \\
$6 / 4 / 92$ & $17801 \mathrm{~F} 3$ & 8.775 \\
\hline
\end{tabular}

Table A-16. Percent of Possible Daytime Data Collected and Percent Possibly Passing SERI QC

\begin{tabular}{|r|rrrrrrrrrrrr|}
\hline Year & Jan & Feb & Mar & Apr & May & June & July & Aug & Sept & Oct & Nov & Dec \\
\hline \multirow{2}{*}{85} & & & & & & & & & 85 & 15 & 45 & 17 \\
& & & & & & & & & $\mathbf{7 4}$ & $\mathbf{1 4}$ & $\mathbf{3 9}$ & $\mathbf{1 6}$ \\
86 & 86 & 94 & 99 & 100 & 90 & 100 & 1 & 0 & 80 & 57 & 94 & 8 \\
& $\mathbf{8 3}$ & $\mathbf{8 9}$ & $\mathbf{9 6}$ & $\mathbf{9 8}$ & $\mathbf{8 9}$ & $\mathbf{9 7}$ & $\mathbf{1}$ & $\mathbf{0}$ & $\mathbf{8 0}$ & $\mathbf{5 4}$ & $\mathbf{9 2}$ & $\mathbf{8}$ \\
87 & 97 & 100 & 100 & 100 & 100 & 100 & 100 & 98 & 100 & 82 & 96 & 100 \\
& $\mathbf{8 8}$ & $\mathbf{9 7}$ & $\mathbf{9 7}$ & $\mathbf{9 7}$ & $\mathbf{9 7}$ & $\mathbf{9 9}$ & $\mathbf{9 9}$ & $\mathbf{9 5}$ & $\mathbf{9 9}$ & $\mathbf{8 0}$ & $\mathbf{9 2}$ & $\mathbf{9 5}$ \\
88 & 98 & 0 & 0 & 0 & 0 & 0 & 0 & 0 & 0 & 0 & 0 & 0 \\
& $\mathbf{9 4}$ & $\mathbf{0}$ & $\mathbf{0}$ & $\mathbf{0}$ & $\mathbf{0}$ & $\mathbf{0}$ & $\mathbf{0}$ & $\mathbf{0}$ & $\mathbf{0}$ & $\mathbf{0}$ & $\mathbf{0}$ & $\mathbf{0}$ \\
89 & 0 & 0 & 0 & 0 & 93 & 100 & 100 & 100 & 97 & 100 & 100 & 100 \\
& $\mathbf{0}$ & $\mathbf{0}$ & $\mathbf{0}$ & $\mathbf{0}$ & $\mathbf{9 1}$ & $\mathbf{9 8}$ & $\mathbf{9 7}$ & $\mathbf{9 4}$ & $\mathbf{9 5}$ & $\mathbf{9 8}$ & $\mathbf{9 8}$ & $\mathbf{8 9}$ \\
90 & 100 & 97 & 100 & 100 & 100 & 77 & 84 & 99 & 100 & 98 & 97 & 100 \\
& $\mathbf{9 8}$ & $\mathbf{9 5}$ & $\mathbf{9 4}$ & $\mathbf{9 7}$ & $\mathbf{9 5}$ & $\mathbf{7 2}$ & $\mathbf{5 9}$ & $\mathbf{8 9}$ & $\mathbf{9 4}$ & $\mathbf{9 2}$ & $\mathbf{9 2}$ & $\mathbf{9 8}$ \\
91 & 100 & 100 & 100 & 100 & 100 & 0 & 100 & 100 & 100 & 100 & 100 & 100 \\
& $\mathbf{9 5}$ & $\mathbf{9 6}$ & $\mathbf{9 5}$ & $\mathbf{8 7}$ & $\mathbf{8 6}$ & $\mathbf{0}$ & $\mathbf{9 1}$ & $\mathbf{9 1}$ & $\mathbf{9 8}$ & $\mathbf{9 4}$ & $\mathbf{9 8}$ & $\mathbf{9 8}$ \\
92 & 100 & 100 & 100 & 100 & 100 & 74 & 100 & 81 & 100 & 100 & 100 & 100 \\
& $\mathbf{9 4}$ & $\mathbf{9 4}$ & $\mathbf{9 8}$ & $\mathbf{9 9}$ & $\mathbf{9 8}$ & $\mathbf{3 4}$ & $\mathbf{9 9}$ & $\mathbf{7 8}$ & $\mathbf{9 8}$ & $\mathbf{9 8}$ & $\mathbf{9 7}$ & $\mathbf{9 8}$ \\
93 & 100 & 96 & 80 & 100 & $\mathbf{7 2}$ & 99 & 100 & 100 & 100 & 100 & 100 & 100 \\
& $\mathbf{9 7}$ & $\mathbf{8 7}$ & $\mathbf{7 8}$ & $\mathbf{9 8}$ & $\mathbf{6 9}$ & $\mathbf{9 2}$ & $\mathbf{9 2}$ & $\mathbf{9 2}$ & $\mathbf{9 5}$ & $\mathbf{8 9}$ & $\mathbf{8 8}$ & $\mathbf{8 9}$ \\
& & & & & & & & & & & & \\
& & & & & & & & & & &
\end{tabular}

Note: Top number is percentage of possible data collected; boldfaced number is percentage of data passing SERI QC. 


\section{A.9 Diffuse Horizontal Radiation for Elizabeth City State University}

Sensor Instrument: Eppley Laboratory pyranometer, model PSP with a shadowband

Table A-17. Record of Instrument Changes and Calibration Factors

\begin{tabular}{|c|c|c|}
\hline Date & Serial Number & $\begin{array}{c}\text { Calibration Factor } \\
\left(\mu \mathrm{V} / \mathrm{W} / \mathrm{m}^{2}\right)\end{array}$ \\
\hline $9 / 3 / 85$ & $25819 \mathrm{~F} 3$ & 9.982 \\
$10 / 1 / 87$ & $25825 \mathrm{~F} 3$ & 9.804 \\
$5 / 2 / 89$ & $17860 \mathrm{~F} 3$ & 7.553 \\
$8 / 9 / 89$ & $18041 \mathrm{~F} 3$ & 7.312 \\
$2 / 15 / 90$ & $25819 \mathrm{~F} 3$ & 9.339 \\
$6 / 4 / 92$ & $24191 \mathrm{~F} 3$ & 9.223 \\
\hline
\end{tabular}

Table A-18. Percent of Possible Daytime Data Collected and Percent Possibly Passing SERI QC

\begin{tabular}{|r|rrrrrrrrrrrr|}
\hline Year & Jan & Feb & Mar & Apr & May & June & July & Aug & Sept & Oct & Nov & Dec \\
\hline \multirow{2}{*}{85} & & & & & & & & & 85 & 15 & 45 & 17 \\
& & & & & & & & & $\mathbf{8 4}$ & $\mathbf{1 4}$ & $\mathbf{4 1}$ & $\mathbf{1 6}$ \\
86 & 86 & 94 & 99 & 100 & 90 & 100 & 1 & 0 & 80 & 57 & 94 & 8 \\
& $\mathbf{8 4}$ & $\mathbf{9 1}$ & $\mathbf{9 7}$ & $\mathbf{9 8}$ & $\mathbf{8 9}$ & $\mathbf{9 7}$ & $\mathbf{1}$ & $\mathbf{0}$ & $\mathbf{8 0}$ & $\mathbf{5 4}$ & $\mathbf{9 3}$ & $\mathbf{8}$ \\
87 & 97 & 100 & 100 & 100 & 100 & 100 & 100 & 98 & 100 & 82 & 96 & 100 \\
& $\mathbf{9 3}$ & $\mathbf{9 7}$ & $\mathbf{9 7}$ & $\mathbf{9 7}$ & $\mathbf{9 8}$ & $\mathbf{9 8}$ & $\mathbf{9 9}$ & $\mathbf{9 6}$ & $\mathbf{9 9}$ & $\mathbf{8 2}$ & $\mathbf{9 3}$ & $\mathbf{9 8}$ \\
88 & 98 & 0 & 0 & 0 & 0 & 0 & 0 & 0 & 0 & 0 & 0 & 0 \\
& $\mathbf{9 5}$ & $\mathbf{0}$ & $\mathbf{0}$ & $\mathbf{0}$ & $\mathbf{0}$ & $\mathbf{0}$ & $\mathbf{0}$ & $\mathbf{0}$ & $\mathbf{0}$ & $\mathbf{0}$ & $\mathbf{0}$ & $\mathbf{0}$ \\
89 & 0 & 0 & 0 & 0 & 93 & 100 & 100 & 100 & 97 & 100 & 100 & 100 \\
& $\mathbf{0}$ & $\mathbf{0}$ & $\mathbf{0}$ & $\mathbf{0}$ & $\mathbf{9 2}$ & $\mathbf{9 8}$ & $\mathbf{9 7}$ & $\mathbf{9 7}$ & $\mathbf{9 6}$ & $\mathbf{9 8}$ & $\mathbf{9 9}$ & $\mathbf{9 5}$ \\
90 & 100 & 97 & 100 & 100 & 100 & 77 & 84 & 99 & 100 & 98 & 97 & 100 \\
& $\mathbf{9 8}$ & $\mathbf{9 5}$ & $\mathbf{9 4}$ & $\mathbf{9 7}$ & $\mathbf{9 6}$ & $\mathbf{7 2}$ & $\mathbf{5 9}$ & $\mathbf{9 0}$ & $\mathbf{9 4}$ & $\mathbf{9 3}$ & $\mathbf{9 2}$ & $\mathbf{9 9}$ \\
91 & 100 & 100 & 100 & 100 & 100 & 0 & 100 & 100 & 100 & 100 & 100 & 100 \\
& $\mathbf{9 7}$ & $\mathbf{9 6}$ & $\mathbf{9 5}$ & $\mathbf{8 7}$ & $\mathbf{8 6}$ & $\mathbf{0}$ & $\mathbf{9 2}$ & $\mathbf{9 1}$ & $\mathbf{9 8}$ & $\mathbf{9 5}$ & $\mathbf{9 8}$ & $\mathbf{9 8}$ \\
92 & 100 & 100 & 100 & 100 & 100 & 74 & 100 & 81 & 100 & 100 & 100 & 100 \\
& $\mathbf{9 4}$ & $\mathbf{9 4}$ & $\mathbf{9 8}$ & $\mathbf{9 9}$ & $\mathbf{9 8}$ & $\mathbf{3 4}$ & $\mathbf{9 9}$ & $\mathbf{7 8}$ & $\mathbf{9 9}$ & $\mathbf{9 9}$ & $\mathbf{9 8}$ & $\mathbf{9 9}$ \\
93 & 100 & 96 & 80 & 100 & $\mathbf{7 2}$ & 99 & 100 & 100 & 100 & 100 & $\mathbf{7 7}$ & 100 \\
& $\mathbf{9 8}$ & $\mathbf{8 7}$ & $\mathbf{7 8}$ & $\mathbf{9 9}$ & $\mathbf{7 0}$ & $\mathbf{9 1}$ & $\mathbf{9 3}$ & $\mathbf{9 2}$ & $\mathbf{9 4}$ & $\mathbf{9 0}$ & $\mathbf{6 9}$ & $\mathbf{9 1}$ \\
& & & & & & & & & & & & \\
& & & & & & & & & & &
\end{tabular}

Note: Top number is percentage of possible data collected; boldfaced number is percentage of data passing SERI QC. 


\section{A.10 Direct Normal Radiation for Elizabeth City State University}

Sensor Instrument: Eppley Laboratory pyrheliometer, model NIP

Table A-19. Record of Instrument Changes and Calibration Factors

\begin{tabular}{|c|c|c|}
\hline Date & Serial Number & $\begin{array}{c}\text { Calibration Factor } \\
\left(\mu \mathrm{V} / \mathrm{W} / \mathrm{m}^{2}\right)\end{array}$ \\
\hline $3 / 1 / 90$ & $25792 \mathrm{E} 6$ & 7.682 \\
$6 / 4 / 92$ & $19791 \mathrm{E} 6$ & 8.562 \\
\hline
\end{tabular}

Table A-20. Percent of Possible Daytime Data Collected and Percent Possibly Passing SERI QC

\begin{tabular}{|c|c|c|c|c|c|c|c|c|c|c|c|c|}
\hline Year & $\operatorname{Jan}$ & Feb & Mar & Apr & May & June & July & Aug & Sept & Oct & Nov & $\overline{\mathrm{Dec}}$ \\
\hline 90 & & & $\begin{array}{r}100 \\
94\end{array}$ & $\begin{array}{r}100 \\
97\end{array}$ & $\begin{array}{r}100 \\
96\end{array}$ & $\begin{array}{l}77 \\
72\end{array}$ & $\begin{array}{l}84 \\
\mathbf{5 9}\end{array}$ & $\begin{array}{l}99 \\
90\end{array}$ & $\begin{array}{r}100 \\
94\end{array}$ & $\begin{array}{l}98 \\
93\end{array}$ & $\begin{array}{l}97 \\
91\end{array}$ & $\begin{array}{r}100 \\
97\end{array}$ \\
\hline 91 & $\begin{array}{r}100 \\
95\end{array}$ & $\begin{array}{r}100 \\
96\end{array}$ & $\begin{array}{r}100 \\
96\end{array}$ & $\begin{array}{r}100 \\
89\end{array}$ & $\begin{array}{r}100 \\
86\end{array}$ & $\begin{array}{l}0 \\
\mathbf{0}\end{array}$ & $\begin{array}{r}100 \\
94\end{array}$ & $\begin{array}{r}100 \\
93\end{array}$ & $\begin{array}{r}100 \\
\mathbf{9 8}\end{array}$ & $\begin{array}{r}100 \\
95\end{array}$ & $\begin{array}{r}100 \\
98\end{array}$ & $\begin{array}{r}100 \\
98\end{array}$ \\
\hline 92 & $\begin{array}{r}100 \\
94\end{array}$ & $\begin{array}{r}100 \\
94\end{array}$ & $\begin{array}{r}100 \\
\mathbf{9 8}\end{array}$ & $\begin{array}{r}100 \\
99\end{array}$ & $\begin{array}{r}100 \\
99\end{array}$ & $\begin{array}{l}74 \\
34\end{array}$ & $\begin{array}{l}100 \\
100\end{array}$ & $\begin{array}{l}81 \\
79\end{array}$ & $\begin{array}{r}100 \\
99\end{array}$ & $\begin{array}{r}100 \\
99\end{array}$ & $\begin{array}{r}100 \\
99\end{array}$ & $\begin{array}{r}100 \\
99\end{array}$ \\
\hline 93 & $\begin{array}{r}100 \\
99\end{array}$ & $\begin{array}{l}96 \\
87\end{array}$ & $\begin{array}{l}80 \\
78\end{array}$ & $\begin{array}{r}100 \\
99\end{array}$ & $\begin{array}{l}72 \\
70\end{array}$ & $\begin{array}{l}99 \\
92\end{array}$ & $\begin{array}{r}100 \\
92\end{array}$ & $\begin{array}{r}100 \\
92\end{array}$ & $\begin{array}{r}100 \\
95\end{array}$ & $\begin{array}{r}100 \\
91\end{array}$ & $\begin{array}{r}100 \\
\mathbf{9 0}\end{array}$ & $\begin{array}{r}100 \\
91\end{array}$ \\
\hline
\end{tabular}

Note: Top number is percentage of possible data collected; boldfaced number is percentage of data passing SERI QC. 


\section{A.11 Direct Normal Radiation from Measurements and/or Calculations for Elizabeth City State University}

Table A-21. Percent of Possible Daytime Data Passing SERI QC

\begin{tabular}{|r|rrrrrrrrrrrrr|}
\hline Year & Jan & Feb & Mar & Apr & May & June & July & Aug & Sept & Oct & Nov & Dec \\
\hline 85 & & & & & & & & & 74 & 14 & 39 & 16 \\
86 & 83 & 89 & 96 & 98 & 89 & 97 & 1 & 0 & 80 & 54 & 92 & 8 \\
87 & 88 & 97 & 97 & 97 & 97 & 98 & 99 & 95 & 99 & 80 & 92 & 95 \\
88 & 94 & 0 & 0 & 0 & 0 & 0 & 0 & 0 & 0 & 0 & 0 & 0 \\
89 & 0 & 0 & 0 & 0 & 91 & 98 & 97 & 94 & 95 & 98 & 98 & 89 \\
90 & 98 & 95 & 94 & 97 & 96 & 72 & 59 & 91 & 94 & 93 & 92 & 99 \\
91 & 97 & 96 & 96 & 89 & 86 & 0 & 94 & 93 & 98 & 95 & 98 & 98 \\
92 & 94 & 94 & 98 & 99 & 99 & 34 & 100 & 79 & 99 & 99 & 99 & 99 \\
93 & 99 & 87 & 78 & 99 & 70 & 92 & 93 & 92 & 95 & 91 & 90 & 91 \\
& & & & & & & & & & & & \\
\hline
\end{tabular}

Table A-22. Percent of Possible Daytime Data Passing SERI QC and Shadowband Alignment Tests

\begin{tabular}{|r|rrrrrrrrrrrr|}
\hline Year & Jan & Feb & Mar & Apr & May & June & July & Aug & Sept & Oct & Nov & Dec \\
\hline 85 & & & & & & & & & 69 & 14 & 39 & 16 \\
86 & 83 & 82 & 96 & 98 & 89 & 97 & 1 & 0 & 80 & 54 & 92 & 8 \\
87 & 88 & 97 & 97 & 97 & 97 & 98 & 99 & 95 & 99 & 80 & 92 & 95 \\
88 & 94 & 0 & 0 & 0 & 0 & 0 & 0 & 0 & 0 & 0 & 0 & 0 \\
89 & 0 & 0 & 0 & 0 & 90 & 98 & 97 & 94 & 95 & 98 & 98 & 89 \\
90 & 98 & 93 & 94 & 97 & 96 & 72 & 59 & 91 & 94 & 93 & 92 & 99 \\
91 & 97 & 96 & 96 & 89 & 86 & 0 & 94 & 93 & 98 & 95 & 98 & 98 \\
92 & 94 & 94 & 98 & 99 & 99 & 34 & 100 & 79 & 99 & 99 & 99 & 99 \\
93 & 99 & 87 & 78 & 99 & 70 & 92 & 92 & 92 & 95 & 91 & 90 & 91 \\
& & & & & & & & & & & & \\
\hline
\end{tabular}




\section{A.12 Global Horizontal Radiation for Mississippi Valley State University}

Sensor Instrument: Eppley Laboratory pyranometer, model PSP

Table A-23. Record of Instrument Changes and Calibration Factors

\begin{tabular}{|c|c|c|}
\hline Date & Serial Number & $\begin{array}{c}\text { Calibration Factor } \\
\left(\mu \mathrm{V} / \mathrm{W} / \mathrm{m}^{2}\right)\end{array}$ \\
\hline $7 / 1 / 85$ & $18038 \mathrm{~F} 3$ & 9.310 \\
$10 / 1 / 86$ & $25951 \mathrm{~F} 3$ & 8.252 \\
$11 / 20 / 89$ & $18036 \mathrm{~F} 3$ & 7.601 \\
$6 / 15 / 92$ & $18038 \mathrm{~F} 3$ & 8.935 \\
\hline
\end{tabular}

Table A-24. Percent of Possible Daytime Data Collected and Percent Possibly Passing SERI QC

\begin{tabular}{|c|rrrrrrrrrrrr|}
\hline Year & Jan & Feb & Mar & Apr & May & June & July & Aug & Sept & Oct & Nov & Dec \\
\hline 85 & & & & & & & 100 & 100 & 100 & 100 & 100 & 100 \\
& & & & & & & $\mathbf{9 9}$ & $\mathbf{9 8}$ & $\mathbf{9 9}$ & $\mathbf{9 6}$ & $\mathbf{9 8}$ & $\mathbf{9 3}$ \\
86 & 100 & 100 & 100 & 100 & 100 & 100 & 100 & 100 & 100 & 99 & 100 & 100 \\
& $\mathbf{9 9}$ & $\mathbf{9 3}$ & $\mathbf{9 7}$ & $\mathbf{9 9}$ & $\mathbf{9 5}$ & $\mathbf{9 9}$ & $\mathbf{9 9}$ & $\mathbf{9 9}$ & $\mathbf{1 0 0}$ & $\mathbf{9 4}$ & $\mathbf{9 1}$ & $\mathbf{8 9}$ \\
87 & 100 & 100 & 100 & 100 & 100 & 100 & 100 & 100 & 100 & 100 & 100 & 100 \\
& $\mathbf{9 0}$ & $\mathbf{8 5}$ & $\mathbf{9 4}$ & $\mathbf{9 9}$ & $\mathbf{9 4}$ & $\mathbf{9 8}$ & $\mathbf{9 8}$ & $\mathbf{9 8}$ & $\mathbf{9 9}$ & $\mathbf{1 0 0}$ & $\mathbf{9 7}$ & $\mathbf{9 2}$ \\
88 & 100 & 10 & 99 & 100 & 99 & 100 & 100 & 100 & 94 & 100 & 100 & 100 \\
& $\mathbf{9 5}$ & $\mathbf{8}$ & $\mathbf{8 9}$ & $\mathbf{9 6}$ & $\mathbf{9 7}$ & $\mathbf{1 0 0}$ & $\mathbf{9 6}$ & $\mathbf{9 9}$ & $\mathbf{9 4}$ & $\mathbf{9 6}$ & $\mathbf{9 8}$ & $\mathbf{9 4}$ \\
89 & 100 & 100 & 100 & 99 & 100 & 100 & 100 & 100 & 99 & 99 & 99 & 99 \\
& $\mathbf{8 4}$ & $\mathbf{8 6}$ & $\mathbf{8 9}$ & $\mathbf{9 6}$ & $\mathbf{9 2}$ & $\mathbf{9 6}$ & $\mathbf{9 9}$ & $\mathbf{1 0 0}$ & $\mathbf{9 3}$ & $\mathbf{9 7}$ & $\mathbf{5 4}$ & $\mathbf{8 5}$ \\
90 & 100 & 100 & 100 & 100 & 100 & 97 & 100 & 98 & 100 & 100 & 100 & 100 \\
& $\mathbf{8 6}$ & $\mathbf{9 6}$ & $\mathbf{8 8}$ & $\mathbf{9 3}$ & $\mathbf{9 2}$ & $\mathbf{9 6}$ & $\mathbf{9 9}$ & $\mathbf{9 8}$ & $\mathbf{9 9}$ & $\mathbf{9 8}$ & $\mathbf{9 7}$ & $\mathbf{8 5}$ \\
91 & 100 & 100 & 99 & 99 & 100 & 100 & 100 & 100 & 100 & 100 & 100 & 100 \\
& $\mathbf{8 8}$ & $\mathbf{8 8}$ & $\mathbf{9 0}$ & $\mathbf{8 5}$ & $\mathbf{9 4}$ & $\mathbf{9 9}$ & $\mathbf{9 8}$ & $\mathbf{9 8}$ & $\mathbf{9 8}$ & $\mathbf{9 9}$ & $\mathbf{9 8}$ & $\mathbf{8 9}$ \\
92 & 100 & 98 & 100 & 100 & 100 & 88 & 82 & 100 & 0 & 81 & 97 & 94 \\
& $\mathbf{9 1}$ & $\mathbf{9 0}$ & $\mathbf{9 5}$ & $\mathbf{9 9}$ & $\mathbf{9 8}$ & $\mathbf{8 6}$ & $\mathbf{8 2}$ & $\mathbf{9 9}$ & $\mathbf{0}$ & $\mathbf{8 0}$ & $\mathbf{9 3}$ & $\mathbf{7 4}$ \\
93 & 91 & 99 & 92 & 100 & 100 & 100 & 100 & 100 & 100 & 100 & 100 & 93 \\
& $\mathbf{8 9}$ & $\mathbf{9 7}$ & $\mathbf{8 9}$ & $\mathbf{9 5}$ & $\mathbf{9 8}$ & $\mathbf{9 9}$ & $\mathbf{9 9}$ & $\mathbf{9 8}$ & $\mathbf{9 8}$ & $\mathbf{9 7}$ & $\mathbf{9 4}$ & $\mathbf{8 7}$ \\
& & & & & & & & & & & & \\
& & & & & & & & & & &
\end{tabular}

Note: Top number is percentage of possible data collected; boldfaced number is percentage of data passing SERI QC. 


\section{A.13 Diffuse Horizontal Radiation for Mississippi Valley State University}

Sensor Instrument: Eppley Laboratory pyranometer, model PSP with a shadowband

Table A-25. Record of Instrument Changes and Calibration Factors

\begin{tabular}{|c|c|c|}
\hline Date & Serial Number & $\begin{array}{c}\text { Calibration Factor } \\
\left(\mu \mathrm{V} / \mathrm{W} / \mathrm{m}^{2}\right)\end{array}$ \\
\hline $7 / 1 / 85$ & $25765 \mathrm{~F} 3$ & 9.880 \\
$10 / 1 / 86$ & $25818 \mathrm{~F} 3$ & 9.526 \\
$11 / 20 / 89$ & $10839 \mathrm{~F} 3$ & 7.969 \\
$6 / 15 / 92$ & $25818 \mathrm{~F} 3$ & 9.487 \\
\hline
\end{tabular}

Table A-26. Percent of Possible Daytime Data Collected and Percent Possibly Passing SERI QC

\begin{tabular}{|r|rrrrrrrrrrrr|}
\hline Year & Jan & Feb & Mar & Apr & May & June & July & Aug & Sept & Oct & Nov & Dec \\
\hline \multirow{2}{*}{85} & & & & & & & 100 & 100 & 100 & 100 & 100 & 100 \\
& & & & & & & $\mathbf{9 9}$ & $\mathbf{9 8}$ & $\mathbf{9 9}$ & $\mathbf{9 7}$ & $\mathbf{9 8}$ & $\mathbf{9 3}$ \\
86 & 100 & 100 & 100 & 100 & 100 & 100 & 100 & 100 & 100 & 99 & 100 & 100 \\
& $\mathbf{9 9}$ & $\mathbf{9 4}$ & $\mathbf{9 8}$ & $\mathbf{1 0 0}$ & $\mathbf{9 6}$ & $\mathbf{9 9}$ & $\mathbf{1 0 0}$ & $\mathbf{9 9}$ & $\mathbf{1 0 0}$ & $\mathbf{9 5}$ & $\mathbf{9 4}$ & $\mathbf{8 9}$ \\
87 & 100 & 100 & 100 & 100 & 100 & 100 & 100 & 100 & 100 & 100 & 100 & 100 \\
& $\mathbf{9 1}$ & $\mathbf{8 8}$ & $\mathbf{9 5}$ & $\mathbf{9 9}$ & $\mathbf{9 5}$ & $\mathbf{9 9}$ & $\mathbf{9 9}$ & $\mathbf{9 9}$ & $\mathbf{9 9}$ & $\mathbf{1 0 0}$ & $\mathbf{9 8}$ & $\mathbf{9 5}$ \\
88 & 100 & 10 & 99 & 100 & 99 & 100 & 100 & 100 & 94 & 100 & 100 & 100 \\
& $\mathbf{9 6}$ & $\mathbf{8}$ & $\mathbf{9 0}$ & $\mathbf{9 7}$ & $\mathbf{9 7}$ & $\mathbf{1 0 0}$ & $\mathbf{9 8}$ & $\mathbf{9 9}$ & $\mathbf{9 4}$ & $\mathbf{9 8}$ & $\mathbf{9 8}$ & $\mathbf{9 5}$ \\
89 & 100 & 100 & 100 & 99 & 100 & 100 & 100 & 100 & 99 & 99 & 99 & 99 \\
& $\mathbf{8 8}$ & $\mathbf{9 0}$ & $\mathbf{9 1}$ & $\mathbf{9 7}$ & $\mathbf{9 4}$ & $\mathbf{9 7}$ & $\mathbf{9 9}$ & $\mathbf{1 0 0}$ & $\mathbf{9 5}$ & $\mathbf{9 8}$ & $\mathbf{9 5}$ & $\mathbf{8 7}$ \\
90 & 100 & 100 & 100 & 100 & 100 & 97 & 100 & 98 & 100 & 100 & 100 & 100 \\
& $\mathbf{8 8}$ & $\mathbf{9 6}$ & $\mathbf{9 0}$ & $\mathbf{9 5}$ & $\mathbf{9 5}$ & $\mathbf{9 6}$ & $\mathbf{1 0 0}$ & $\mathbf{9 8}$ & $\mathbf{9 9}$ & $\mathbf{9 8}$ & $\mathbf{9 8}$ & $\mathbf{8 9}$ \\
91 & 100 & 100 & 99 & 99 & 100 & 100 & 100 & 100 & 100 & 100 & 100 & 100 \\
& $\mathbf{8 8}$ & $\mathbf{9 3}$ & $\mathbf{9 1}$ & $\mathbf{8 9}$ & $\mathbf{9 4}$ & $\mathbf{9 9}$ & $\mathbf{9 9}$ & $\mathbf{9 9}$ & $\mathbf{9 8}$ & $\mathbf{9 9}$ & $\mathbf{9 9}$ & $\mathbf{9 1}$ \\
92 & 100 & 98 & 100 & 100 & 100 & 88 & 82 & 100 & 0 & 81 & 97 & 94 \\
& $\mathbf{9 2}$ & $\mathbf{9 2}$ & $\mathbf{9 6}$ & $\mathbf{9 9}$ & $\mathbf{9 9}$ & $\mathbf{8 6}$ & $\mathbf{8 2}$ & $\mathbf{1 0 0}$ & $\mathbf{0}$ & $\mathbf{8 0}$ & $\mathbf{9 3}$ & $\mathbf{7 8}$ \\
93 & 91 & 99 & 92 & 100 & 100 & 100 & 100 & 100 & 100 & 100 & 100 & 93 \\
& $\mathbf{8 9}$ & $\mathbf{9 7}$ & $\mathbf{8 9}$ & $\mathbf{9 5}$ & $\mathbf{9 8}$ & $\mathbf{9 9}$ & $\mathbf{9 9}$ & $\mathbf{9 8}$ & $\mathbf{9 8}$ & $\mathbf{9 7}$ & $\mathbf{9 4}$ & $\mathbf{8 7}$ \\
& & & & & & & & & & & & \\
\hline
\end{tabular}

Note: Top number is percentage of possible data collected; boldfaced number is percentage of data passing SERI QC. 


\section{A.14 Direct Normal Radiation for Mississippi Valley State University}

Sensor Instrument: Eppley Laboratory pyrheliometer, model NIP

Table A-27. Record of Instrument Changes and Calibration Factors

\begin{tabular}{|c|c|c|}
\hline Date & Serial Number & $\begin{array}{c}\text { Calibration Factor } \\
\left(\mu \mathrm{V} / \mathrm{W} / \mathrm{m}^{2}\right)\end{array}$ \\
\hline $1 / 1 / 93$ & $17830 \mathrm{E} 6$ & 8.218 \\
\hline
\end{tabular}

Table A-28. Percent of Possible Daytime Data Collected and Percent Possibly Passing SERI QC

\begin{tabular}{|c|rrrrrrrrrrrr|}
\hline Year & Jan & Feb & Mar & Apr & May & June & July & Aug & Sept & Oct & Nov & Dec \\
\hline 93 & 91 & 99 & 92 & 100 & 100 & 100 & 100 & 100 & 100 & 100 & 100 & 93 \\
& $\mathbf{8 9}$ & $\mathbf{9 8}$ & $\mathbf{9 0}$ & $\mathbf{9 8}$ & $\mathbf{9 9}$ & $\mathbf{9 9}$ & $\mathbf{9 9}$ & $\mathbf{9 9}$ & $\mathbf{9 8}$ & $\mathbf{9 7}$ & $\mathbf{9 5}$ & $\mathbf{8 8}$ \\
& & & & & & & & & & & & \\
\hline
\end{tabular}

Note: Top number is percentage of possible data collected; boldfaced number is percentage of data passing SERI QC. 


\section{A.15 Direct Normal Radiation from Measurements and/or Calculations for Mississippi Valley State University}

Table A-29. Percent of Possible Daytime Data Passing SERI QC

\begin{tabular}{|c|rrrrrrrrrrrr|}
\hline Year & Jan & Feb & Mar & Apr & May & June & July & Aug & Sept & Oct & Nov & Dec \\
\hline 85 & & & & & & & 98 & 97 & 99 & 96 & 98 & 93 \\
86 & 99 & 93 & 97 & 99 & 95 & 99 & 99 & 99 & 100 & 94 & 91 & 89 \\
87 & 90 & 85 & 94 & 99 & 94 & 98 & 98 & 98 & 99 & 100 & 97 & 92 \\
88 & 95 & 8 & 89 & 96 & 97 & 100 & 96 & 99 & 94 & 96 & 98 & 94 \\
89 & 84 & 86 & 89 & 96 & 92 & 96 & 98 & 100 & 93 & 97 & 54 & 84 \\
90 & 86 & 96 & 88 & 93 & 92 & 96 & 99 & 98 & 98 & 98 & 97 & 85 \\
91 & 88 & 88 & 90 & 85 & 94 & 99 & 98 & 98 & 98 & 99 & 98 & 89 \\
92 & 91 & 90 & 95 & 99 & 98 & 86 & 82 & 99 & 0 & 80 & 93 & 74 \\
93 & 89 & 98 & 90 & 98 & 99 & 99 & 99 & 99 & 98 & 97 & 95 & 88 \\
& & & & & & & & & & & & \\
\hline
\end{tabular}

Table A-30. Percent of Possible Daytime Data Passing SERI QC and Shadowband Alignment Tests

\begin{tabular}{|c|rrrrrrrrrrrrr|}
\hline Year & Jan & Feb & Mar & Apr & May & June & July & Aug & Sept & Oct & Nov & Dec \\
\hline 85 & & & & & & & 98 & 97 & 99 & 95 & 98 & 93 \\
86 & 99 & 89 & 97 & 99 & 95 & 98 & 99 & 99 & 100 & 93 & 91 & 88 \\
87 & 85 & 78 & 82 & 99 & 94 & 98 & 98 & 97 & 94 & 94 & 94 & 92 \\
88 & 84 & 8 & 82 & 95 & 96 & 99 & 95 & 98 & 89 & 89 & 95 & 89 \\
89 & 77 & 67 & 57 & 84 & 80 & 90 & 96 & 98 & 82 & 92 & 50 & 84 \\
90 & 86 & 96 & 88 & 93 & 92 & 96 & 99 & 98 & 97 & 96 & 94 & 85 \\
91 & 88 & 87 & 87 & 83 & 94 & 99 & 98 & 98 & 98 & 99 & 98 & 89 \\
92 & 91 & 86 & 89 & 98 & 98 & 86 & 81 & 99 & 0 & 80 & 87 & 62 \\
93 & 89 & 98 & 90 & 98 & 99 & 99 & 99 & 99 & 98 & 97 & 95 & 88 \\
& & & & & & & & & & & & \\
\hline
\end{tabular}




\section{A.16 Global Horizontal Radiation for South Carolina State University}

Sensor Instrument: Eppley Laboratory pyranometer, model PSP

Table A-31. Record of Instrument Changes and Calibration Factors

\begin{tabular}{|c|c|c|}
\hline Date & Serial Number & $\begin{array}{c}\text { Calibration Factor } \\
\left(\mu \mathrm{V} / \mathrm{W} / \mathrm{m}^{2}\right)\end{array}$ \\
\hline $8 / 1 / 85$ & $17897 \mathrm{~F} 3$ & 8.250 \\
$11 / 11 / 86$ & $25765 \mathrm{~F} 3$ & 9.346 \\
$12 / 19 / 89$ & $18078 \mathrm{~F} 3$ & 9.618 \\
$6 / 8 / 92$ & $17897 \mathrm{~F} 3$ & 7.627 \\
\hline
\end{tabular}

Table A-32. Percent of Possible Daytime Data Collected and Percent Possibly Passing SERI QC

\begin{tabular}{|r|rrrrrrrrrrrr|}
\hline Year & Jan & Feb & Mar & Apr & May & June & July & Aug & Sept & Oct & Nov & Dec \\
\hline \multirow{2}{*}{85} & & & & & & & & 98 & 100 & 100 & 7 & 78 \\
& & & & & & & & $\mathbf{9 5}$ & $\mathbf{1 0 0}$ & $\mathbf{9 7}$ & $\mathbf{6}$ & $\mathbf{7 4}$ \\
86 & 96 & 100 & 100 & 91 & 93 & 100 & 97 & 100 & 95 & 6 & 79 & 100 \\
& $\mathbf{9 4}$ & $\mathbf{9 5}$ & $\mathbf{9 8}$ & $\mathbf{9 1}$ & $\mathbf{9 3}$ & $\mathbf{9 8}$ & $\mathbf{9 7}$ & $\mathbf{9 9}$ & $\mathbf{9 5}$ & $\mathbf{6}$ & $\mathbf{7 4}$ & $\mathbf{9 6}$ \\
87 & 99 & 100 & 97 & 97 & 91 & 94 & 36 & 86 & 99 & 98 & 100 & 99 \\
& $\mathbf{9 4}$ & $\mathbf{8 8}$ & $\mathbf{9 1}$ & $\mathbf{9 6}$ & $\mathbf{9 0}$ & $\mathbf{9 2}$ & $\mathbf{3 6}$ & $\mathbf{8 3}$ & $\mathbf{9 7}$ & $\mathbf{9 8}$ & $\mathbf{9 7}$ & $\mathbf{9 6}$ \\
88 & 34 & 0 & 24 & 100 & 100 & 100 & 100 & 99 & 100 & 100 & 100 & 100 \\
& $\mathbf{3 0}$ & $\mathbf{0}$ & $\mathbf{2 4}$ & $\mathbf{9 7}$ & $\mathbf{9 9}$ & $\mathbf{9 7}$ & $\mathbf{9 9}$ & $\mathbf{9 7}$ & $\mathbf{9 7}$ & $\mathbf{9 8}$ & $\mathbf{9 9}$ & $\mathbf{9 8}$ \\
89 & 100 & 100 & 100 & 100 & 100 & 100 & 98 & 100 & 97 & 100 & 100 & 99 \\
& $\mathbf{9 6}$ & $\mathbf{9 4}$ & $\mathbf{9 1}$ & $\mathbf{9 4}$ & $\mathbf{1 0 0}$ & $\mathbf{9 8}$ & $\mathbf{9 6}$ & $\mathbf{9 9}$ & $\mathbf{9 6}$ & $\mathbf{9 9}$ & $\mathbf{9 6}$ & $\mathbf{9 1}$ \\
90 & 100 & 90 & 100 & 100 & 100 & 100 & 100 & 100 & 100 & 70 & 99 & 100 \\
& $\mathbf{9 9}$ & $\mathbf{8 8}$ & $\mathbf{9 5}$ & $\mathbf{9 8}$ & $\mathbf{9 8}$ & $\mathbf{9 8}$ & $\mathbf{1 0 0}$ & $\mathbf{9 9}$ & $\mathbf{9 9}$ & $\mathbf{6 4}$ & $\mathbf{9 6}$ & $\mathbf{9 3}$ \\
91 & 100 & 100 & 3 & 39 & 100 & 100 & 92 & 100 & 100 & 100 & 100 & 100 \\
& $\mathbf{9 0}$ & $\mathbf{9 6}$ & $\mathbf{3}$ & $\mathbf{3 8}$ & $\mathbf{9 7}$ & $\mathbf{9 9}$ & $\mathbf{8 9}$ & $\mathbf{9 9}$ & $\mathbf{9 9}$ & $\mathbf{9 9}$ & $\mathbf{9 5}$ & $\mathbf{9 6}$ \\
92 & 100 & 100 & 100 & 100 & 100 & 92 & 100 & 100 & 100 & 100 & 99 & 100 \\
& $\mathbf{9 7}$ & $\mathbf{9 5}$ & $\mathbf{9 5}$ & $\mathbf{9 7}$ & $\mathbf{9 9}$ & $\mathbf{8 9}$ & $\mathbf{9 7}$ & $\mathbf{9 9}$ & $\mathbf{9 9}$ & $\mathbf{9 9}$ & $\mathbf{9 2}$ & $\mathbf{9 4}$ \\
93 & 100 & 100 & 94 & 99 & 93 & 100 & 98 & 100 & 100 & 100 & 100 & 100 \\
& $\mathbf{9 2}$ & $\mathbf{9 6}$ & $\mathbf{8 8}$ & $\mathbf{9 6}$ & $\mathbf{9 0}$ & $\mathbf{9 7}$ & $\mathbf{9 7}$ & $\mathbf{9 7}$ & $\mathbf{9 5}$ & $\mathbf{9 7}$ & $\mathbf{9 6}$ & $\mathbf{9 5}$ \\
& & & & & & & & & & & & \\
& & & & & & & & & & &
\end{tabular}

Note: Top number is percentage of possible data collected; boldfaced number is percentage of data passing SERI QC. 


\section{A.17 Diffuse Horizontal Radiation for South Carolina State University}

Sensor Instrument: Eppley Laboratory pyranometer, model PSP with a shadowband

Table A-33. Record of Instrument Changes and Calibration Factors

\begin{tabular}{|c|c|c|}
\hline Date & Serial Number & $\begin{array}{c}\text { Calibration Factor } \\
\left(\mu \mathrm{V} / \mathrm{W} / \mathrm{m}^{2}\right)\end{array}$ \\
\hline $8 / 1 / 85$ & $25824 \mathrm{~F} 3$ & 10.700 \\
$11 / 11 / 86$ & $18038 \mathrm{~F} 3$ & 8.756 \\
$12 / 19 / 89$ & $17798 \mathrm{~F} 3$. & 7.893 \\
$6 / 8 / 92$ & $25824 \mathrm{~F} 3$ & 10.247 \\
\hline
\end{tabular}

Table A-34. Percent of Possible Daytime Data Collected and Percent Possibly Passing SERI QC

\begin{tabular}{|r|rrrrrrrrrrrr|}
\hline Year & Jan & Feb & Mar & Apr & May & June & July & Aug & Sept & Oct & Nov & Dec \\
\hline \multirow{2}{*}{85} & & & & & & & & 98 & 100 & 100 & 7 & 78 \\
& & & & & & & & $\mathbf{9 6}$ & $\mathbf{1 0 0}$ & $\mathbf{9 7}$ & $\mathbf{6}$ & $\mathbf{7 5}$ \\
86 & 96 & 100 & 100 & 91 & 93 & 100 & 97 & 100 & 95 & 6 & 79 & 100 \\
& $\mathbf{9 5}$ & $\mathbf{9 3}$ & $\mathbf{9 8}$ & $\mathbf{9 1}$ & $\mathbf{9 3}$ & $\mathbf{9 8}$ & $\mathbf{9 7}$ & $\mathbf{9 9}$ & $\mathbf{9 3}$ & $\mathbf{6}$ & $\mathbf{6 0}$ & $\mathbf{9 6}$ \\
87 & 99 & 100 & 97 & 97 & 91 & 94 & 36 & 86 & 99 & 98 & 100 & 99 \\
& $\mathbf{9 6}$ & $\mathbf{8 7}$ & $\mathbf{7 7}$ & $\mathbf{9 1}$ & $\mathbf{9 0}$ & $\mathbf{9 3}$ & $\mathbf{3 6}$ & $\mathbf{8 4}$ & $\mathbf{9 7}$ & $\mathbf{9 8}$ & $\mathbf{9 8}$ & $\mathbf{9 6}$ \\
88 & 34 & 0 & 24 & 100 & 100 & 100 & 100 & 99 & 100 & 100 & 100 & 100 \\
& $\mathbf{3 2}$ & $\mathbf{0}$ & $\mathbf{2 4}$ & $\mathbf{9 7}$ & $\mathbf{1 0 0}$ & $\mathbf{9 8}$ & $\mathbf{1 0 0}$ & $\mathbf{9 7}$ & $\mathbf{9 7}$ & $\mathbf{1 0 0}$ & $\mathbf{9 9}$ & $\mathbf{9 8}$ \\
89 & 100 & 100 & 100 & 100 & 100 & 100 & 98 & 100 & 97 & 100 & 100 & 99 \\
& $\mathbf{9 7}$ & $\mathbf{9 3}$ & $\mathbf{9 7}$ & $\mathbf{8 9}$ & $\mathbf{1 0 0}$ & $\mathbf{9 8}$ & $\mathbf{9 7}$ & $\mathbf{1 0 0}$ & $\mathbf{9 7}$ & $\mathbf{9 9}$ & $\mathbf{9 6}$ & $\mathbf{9 3}$ \\
90 & 100 & 100 & 100 & 100 & 100 & 100 & 100 & 100 & 100 & 70 & 99 & 100 \\
& $\mathbf{9 9}$ & $\mathbf{9 7}$ & $\mathbf{8 6}$ & $\mathbf{8 3}$ & $\mathbf{8 6}$ & $\mathbf{9 9}$ & $\mathbf{1 0 0}$ & $\mathbf{9 9}$ & $\mathbf{9 9}$ & $\mathbf{6 6}$ & $\mathbf{9 6}$ & $\mathbf{9 6}$ \\
91 & 100 & 100 & 3 & 39 & 100 & 100 & 92 & 100 & 100 & 100 & 100 & 100 \\
& $\mathbf{9 5}$ & $\mathbf{9 5}$ & $\mathbf{3}$ & $\mathbf{3 6}$ & $\mathbf{9 4}$ & $\mathbf{1 0 0}$ & $\mathbf{9 1}$ & $\mathbf{9 9}$ & $\mathbf{9 9}$ & $\mathbf{1 0 0}$ & $\mathbf{9 6}$ & $\mathbf{9 7}$ \\
92 & 100 & 100 & 100 & 100 & 100 & 92 & 100 & 100 & 100 & 100 & 99 & 100 \\
& $\mathbf{9 8}$ & $\mathbf{9 1}$ & $\mathbf{9 3}$ & $\mathbf{9 0}$ & $\mathbf{9 8}$ & $\mathbf{8 8}$ & $\mathbf{9 8}$ & $\mathbf{1 0 0}$ & $\mathbf{9 9}$ & $\mathbf{1 0 0}$ & $\mathbf{8 2}$ & $\mathbf{9 5}$ \\
93 & 100 & 100 & 94 & 99 & 93 & 100 & 98 & 100 & 100 & 100 & 100 & 100 \\
& $\mathbf{9 3}$ & $\mathbf{9 1}$ & $\mathbf{7 4}$ & $\mathbf{9 1}$ & $\mathbf{6 6}$ & $\mathbf{7 8}$ & $\mathbf{9 2}$ & $\mathbf{8 7}$ & $\mathbf{6 9}$ & $\mathbf{8 2}$ & $\mathbf{9 2}$ & $\mathbf{9 5}$ \\
& & & & & & & & & & & & \\
& & & & & & & & & & & &
\end{tabular}

Note: Top number is percentage of possible data collected; boldfaced number is percentage of data passing SERI QC. 


\section{A.18 Direct Normal Radiation from Calculations for South Carolina State University}

Table A-35. Percent of Possible Daytime Data Passing SERI QC

\begin{tabular}{|r|rrrrrrrrrrrrr|}
\hline Year & Jan & Feb & Mar & Apr & May & June & July & Aug & Sept & Oct & Nov & Dec \\
\hline 85 & & & & & & & & 95 & 100 & 97 & 6 & 74 \\
86 & 94 & 92 & 98 & 91 & 93 & 98 & 97 & 99 & 93 & 6 & 60 & 96 \\
87 & 94 & 83 & 75 & 90 & 90 & 92 & 36 & 83 & 97 & 98 & 97 & 96 \\
88 & 30 & 0 & 24 & 96 & 99 & 96 & 99 & 97 & 96 & 98 & 99 & 98 \\
89 & 96 & 92 & 91 & 86 & 100 & 98 & 96 & 99 & 96 & 99 & 96 & 91 \\
90 & 99 & 87 & 85 & 83 & 85 & 98 & 100 & 99 & 99 & 64 & 96 & 93 \\
91 & 90 & 95 & 3 & 36 & 93 & 99 & 89 & 99 & 99 & 99 & 95 & 96 \\
92 & 97 & 90 & 92 & 90 & 97 & 87 & 97 & 99 & 99 & 99 & 80 & 94 \\
93 & 90 & 90 & 72 & 90 & 66 & 78 & 92 & 87 & 67 & 82 & 92 & 95 \\
& & & & & & & & & & & & \\
\hline
\end{tabular}

Table A-36. Percent of Possible Daytime Data Passing SERI QC and Shadowband Alignment Tests

\begin{tabular}{|r|rrrrrrrrrrrrr|}
\hline Year & Jan & Feb & Mar & Apr & May & June & July & Aug & Sept & Oct & Nov & Dec \\
\hline 85 & & & & & & & & 95 & 100 & 96 & 6 & 74 \\
86 & 94 & 91 & 98 & 91 & 93 & 98 & 97 & 99 & 85 & 6 & 18 & 96 \\
87 & 92 & 47 & 48 & 87 & 86 & 85 & 36 & 79 & 89 & 95 & 93 & 90 \\
88 & 29 & 0 & 24 & 86 & 95 & 93 & 92 & 83 & 65 & 95 & 93 & 97 \\
89 & 80 & 73 & 64 & 70 & 93 & 93 & 87 & 77 & 61 & 87 & 90 & 82 \\
90 & 99 & 71 & 63 & 72 & 76 & 98 & 100 & 99 & 98 & 64 & 95 & 93 \\
91 & 90 & 82 & 3 & 30 & 91 & 99 & 89 & 99 & 95 & 97 & 95 & 96 \\
92 & 94 & 81 & 85 & 78 & 89 & 86 & 97 & 99 & 99 & 98 & 56 & 94 \\
93 & 89 & 77 & 48 & 80 & 52 & 66 & 88 & 71 & 38 & 39 & 85 & 92 \\
& & & & & & & & & & & & \\
\hline
\end{tabular}




\section{A.19 Global Horizontal Radiation for Savannah State College}

Sensor Instrument: Eppley Laboratory pyranometer, model PSP

Table A-37. Record of Instrument Changes and Calibration Factors

\begin{tabular}{|c|c|c|}
\hline Date & Serial Number & $\begin{array}{c}\text { Calibration Factor } \\
\left(\mu \mathrm{V} / \mathrm{W} / \mathrm{m}^{2}\right)\end{array}$ \\
\hline $8 / 29 / 85$ & $25825 \mathrm{~F} 3$ & 10.160 \\
$6 / 24 / 87$ & $25820 \mathrm{~F} 3$ & 9.353 \\
$2 / 6 / 90$ & $17799 \mathrm{~F} 3$ & 8.503 \\
$6 / 9 / 92$ & $25820 \mathrm{~F} 3$ & 9.209 \\
\hline
\end{tabular}

Table A-38. Percent of Possible Daytime Data Collected and Percent Possibly Passing SERI QC

\begin{tabular}{|r|rrrrrrrrrrrr|}
\hline Year & Jan & Feb & Mar & Apr & May & June & July & Aug & Sept & Oct & Nov & Dec \\
\hline \multirow{2}{*}{85} & & & & & & & & 7 & 100 & 92 & 97 & 98 \\
& & & & & & & & $\mathbf{6}$ & $\mathbf{1 0 0}$ & $\mathbf{8 7}$ & $\mathbf{9 6}$ & $\mathbf{9 6}$ \\
86 & 100 & 100 & 100 & 100 & 100 & 89 & 86 & 100 & 100 & 100 & 100 & 100 \\
& $\mathbf{9 6}$ & $\mathbf{9 2}$ & $\mathbf{9 3}$ & $\mathbf{9 9}$ & $\mathbf{9 8}$ & $\mathbf{8 8}$ & $\mathbf{8 0}$ & $\mathbf{9 2}$ & $\mathbf{1 0 0}$ & $\mathbf{9 4}$ & $\mathbf{9 3}$ & $\mathbf{9 4}$ \\
87 & 100 & 100 & 100 & 100 & 100 & 99 & 100 & 100 & 100 & 100 & 100 & 100 \\
& $\mathbf{9 2}$ & $\mathbf{8 5}$ & $\mathbf{8 9}$ & $\mathbf{9 9}$ & $\mathbf{9 8}$ & $\mathbf{9 5}$ & $\mathbf{9 8}$ & $\mathbf{9 8}$ & $\mathbf{9 8}$ & $\mathbf{9 8}$ & $\mathbf{9 7}$ & $\mathbf{9 8}$ \\
88 & 100 & 100 & 97 & 99 & 96 & 100 & 100 & 100 & 100 & 100 & 99 & 100 \\
& $\mathbf{9 4}$ & $\mathbf{9 2}$ & $\mathbf{8 9}$ & $\mathbf{9 3}$ & $\mathbf{9 5}$ & $\mathbf{9 8}$ & $\mathbf{9 9}$ & $\mathbf{9 9}$ & $\mathbf{9 8}$ & $\mathbf{9 9}$ & $\mathbf{9 8}$ & $\mathbf{9 9}$ \\
89 & 100 & 100 & 100 & 100 & 100 & 99 & 82 & 100 & 97 & 81 & 98 & 99 \\
& $\mathbf{1 0 0}$ & $\mathbf{9 7}$ & $\mathbf{8 8}$ & $\mathbf{9 3}$ & $\mathbf{9 7}$ & $\mathbf{9 5}$ & $\mathbf{7 9}$ & $\mathbf{9 3}$ & $\mathbf{9 3}$ & $\mathbf{7 8}$ & $\mathbf{9 7}$ & $\mathbf{9 1}$ \\
90 & 92 & 90 & 100 & 100 & 100 & 100 & 100 & 98 & 100 & 100 & 100 & 100 \\
& $\mathbf{9 0}$ & $\mathbf{8 3}$ & $\mathbf{9 5}$ & $\mathbf{9 7}$ & $\mathbf{9 9}$ & $\mathbf{9 9}$ & $\mathbf{9 8}$ & $\mathbf{9 6}$ & $\mathbf{9 9}$ & $\mathbf{9 5}$ & $\mathbf{9 7}$ & $\mathbf{9 9}$ \\
91 & 100 & 100 & 100 & 100 & 99 & 100 & 100 & 100 & 100 & 100 & 100 & 100 \\
& $\mathbf{9 4}$ & $\mathbf{9 8}$ & $\mathbf{9 3}$ & $\mathbf{9 4}$ & $\mathbf{9 5}$ & $\mathbf{9 5}$ & $\mathbf{9 3}$ & $\mathbf{9 5}$ & $\mathbf{1 0 0}$ & $\mathbf{9 9}$ & $\mathbf{9 7}$ & $\mathbf{9 7}$ \\
92 & 100 & 100 & $\mathbf{7 8}$ & 96 & 99 & 78 & 55 & 99 & 99 & 100 & 92 & 100 \\
& $\mathbf{9 5}$ & $\mathbf{9 4}$ & $\mathbf{7 4}$ & $\mathbf{9 2}$ & $\mathbf{9 5}$ & $\mathbf{7 4}$ & $\mathbf{5 4}$ & $\mathbf{9 4}$ & $\mathbf{9 8}$ & $\mathbf{9 6}$ & $\mathbf{8 7}$ & $\mathbf{9 9}$ \\
93 & 93 & 93 & 100 & 100 & 88 & 87 & 99 & 100 & 100 & 100 & 100 & 0 \\
& $\mathbf{9 7}$ & $\mathbf{8 6}$ & $\mathbf{9 1}$ & $\mathbf{9 7}$ & $\mathbf{8 7}$ & $\mathbf{8 6}$ & $\mathbf{9 7}$ & $\mathbf{9 8}$ & $\mathbf{9 8}$ & $\mathbf{9 4}$ & $\mathbf{9 4}$ & $\mathbf{0}$ \\
& & & & & & & & & & & & \\
& & & & & & & & & & &
\end{tabular}

Note: Top number is percentage of possible data collected; boldfaced number is percentage of data passing SERI QC. 


\section{A.20 Diffuse Horizontal Radiation for Savannah State College}

Sensor Instrument: Eppley Laboratory pyranometer, model PSP with a shadowband

Table A-39. Record of Instrument Changes and Calibration Factors

\begin{tabular}{|c|c|c|}
\hline Date & Serial Number & $\begin{array}{c}\text { Calibration Factor } \\
\left(\mu \mathrm{V} / \mathrm{W} / \mathrm{m}^{2}\right)\end{array}$ \\
\hline $8 / 29 / 85$ & $18041 \mathrm{~F} 3$ & 7.510 \\
$6 / 24 / 87$ & $25782 \mathrm{~F} 3$ & 9.757 \\
$2 / 6 / 90$ & $17862 \mathrm{~F} 3$ & 8.067 \\
$6 / 9 / 92$ & $25783 \mathrm{~F} 3$ & 9.722 \\
\hline
\end{tabular}

Table A-40. Percent of Possible Daytime Data Collected and Percent Possibly Passing SERI QC

\begin{tabular}{|r|rrrrrrrrrrrrr|}
\hline Year & Jan & Feb & Mar & Apr & May & June & July & Aug & Sept & Oct & Nov & Dec \\
\hline 85 & & & & & & & & 7 & 100 & 92 & 97 & 98 \\
& & & & & & & & $\mathbf{6}$ & $\mathbf{1 0 0}$ & $\mathbf{8 8}$ & $\mathbf{9 6}$ & $\mathbf{9 6}$ \\
86 & 100 & 100 & 100 & 100 & 100 & 89 & 86 & 100 & 100 & 100 & 100 & 100 \\
& $\mathbf{9 7}$ & $\mathbf{9 4}$ & $\mathbf{9 4}$ & $\mathbf{1 0 0}$ & $\mathbf{9 8}$ & $\mathbf{8 8}$ & $\mathbf{7 7}$ & $\mathbf{9 5}$ & $\mathbf{1 0 0}$ & $\mathbf{9 6}$ & $\mathbf{9 3}$ & $\mathbf{9 7}$ \\
87 & 100 & 100 & 100 & 100 & 100 & 99 & 100 & 100 & 100 & 100 & 100 & 100 \\
& $\mathbf{9 6}$ & $\mathbf{8 7}$ & $\mathbf{9 1}$ & $\mathbf{9 9}$ & $\mathbf{9 8}$ & $\mathbf{9 6}$ & $\mathbf{9 8}$ & $\mathbf{9 8}$ & $\mathbf{9 9}$ & $\mathbf{9 8}$ & $\mathbf{9 7}$ & $\mathbf{9 9}$ \\
88 & 100 & 100 & 97 & 99 & 58 & 0 & 0 & 0 & 0 & 0 & 14 & 100 \\
& $\mathbf{9 3}$ & $\mathbf{9 5}$ & $\mathbf{9 0}$ & $\mathbf{9 1}$ & $\mathbf{5 7}$ & $\mathbf{0}$ & $\mathbf{0}$ & $\mathbf{0}$ & $\mathbf{0}$ & $\mathbf{0}$ & $\mathbf{1 4}$ & $\mathbf{9 9}$ \\
89 & 100 & 100 & 100 & 100 & 100 & 99 & 82 & 100 & 97 & 81 & 98 & 99 \\
& $\mathbf{1 0 0}$ & $\mathbf{9 7}$ & $\mathbf{9 1}$ & $\mathbf{9 5}$ & $\mathbf{9 8}$ & $\mathbf{9 5}$ & $\mathbf{7 9}$ & $\mathbf{9 2}$ & $\mathbf{9 6}$ & $\mathbf{7 8}$ & $\mathbf{9 7}$ & $\mathbf{9 3}$ \\
90 & 92 & 90 & 100 & 100 & 100 & 100 & 100 & 98 & 100 & 100 & 100 & 100 \\
& $\mathbf{9 1}$ & $\mathbf{8 6}$ & $\mathbf{9 6}$ & $\mathbf{9 7}$ & $\mathbf{9 9}$ & $\mathbf{9 9}$ & $\mathbf{9 8}$ & $\mathbf{9 3}$ & $\mathbf{9 7}$ & $\mathbf{9 7}$ & $\mathbf{9 9}$ & $\mathbf{9 9}$ \\
91 & 100 & 100 & 100 & 100 & 99 & 100 & 100 & 100 & 100 & 100 & 100 & 100 \\
& $\mathbf{9 6}$ & $\mathbf{9 8}$ & $\mathbf{9 4}$ & $\mathbf{9 4}$ & $\mathbf{9 5}$ & $\mathbf{9 7}$ & $\mathbf{9 5}$ & $\mathbf{9 6}$ & $\mathbf{1 0 0}$ & $\mathbf{9 9}$ & $\mathbf{9 9}$ & $\mathbf{9 8}$ \\
92 & 100 & 100 & $\mathbf{7 8}$ & 96 & 99 & 78 & 55 & 99 & 99 & 100 & 92 & 100 \\
& $\mathbf{9 8}$ & $\mathbf{9 5}$ & $\mathbf{7 5}$ & $\mathbf{9 1}$ & $\mathbf{9 6}$ & $\mathbf{7 6}$ & $\mathbf{5 4}$ & $\mathbf{9 6}$ & $\mathbf{9 8}$ & $\mathbf{9 7}$ & $\mathbf{8 7}$ & $\mathbf{9 9}$ \\
93 & 93 & 93 & 100 & 100 & 88 & 87 & 99 & 100 & 100 & 100 & 100 & 0 \\
& $\mathbf{9 0}$ & $\mathbf{8 7}$ & $\mathbf{9 2}$ & $\mathbf{9 7}$ & $\mathbf{8 7}$ & $\mathbf{8 6}$ & $\mathbf{9 8}$ & $\mathbf{9 8}$ & $\mathbf{9 9}$ & $\mathbf{9 5}$ & $\mathbf{9 5}$ & $\mathbf{0}$ \\
& & & & & & & & & & & & \\
& & & & & & & & & & & & &
\end{tabular}

Note: Top number is percentage of possible data collected; boldfaced number is percentage of data passing SERI QC. 


\section{A.21 Direct Normal Radiation from Calculations for Savannah State College}

Table A-41. Percent of Possible Daytime Data Passing SERI QC

\begin{tabular}{|c|rrrrrrrrrrrr|}
\hline Year & Jan & Feb & Mar & Apr & May & June & July & Aug & Sept & Oct & Nov & Dec \\
\hline 85 & & & & & & & & 6 & 100 & 87 & 96 & 96 \\
86 & 96 & 92 & 93 & 99 & 98 & 88 & 76 & 92 & 100 & 94 & 92 & 94 \\
87 & 92 & 83 & 89 & 99 & 98 & 95 & 98 & 98 & 98 & 98 & 97 & 98 \\
88 & 90 & 91 & 89 & 90 & 57 & 0 & 0 & 0 & 0 & 0 & 14 & 99 \\
89 & 100 & 97 & 88 & 93 & 97 & 95 & 79 & 92 & 93 & 78 & 97 & 91 \\
90 & 90 & 83 & 95 & 97 & 99 & 99 & 98 & 93 & 97 & 95 & 97 & 99 \\
91 & 94 & 98 & 93 & 94 & 95 & 95 & 93 & 95 & 100 & 99 & 97 & 97 \\
92 & 95 & 94 & 74 & 91 & 95 & 74 & 54 & 94 & 98 & 96 & 87 & 99 \\
93 & 87 & 86 & 91 & 97 & 87 & 86 & 97 & 98 & 98 & 94 & 94 & 0 \\
& & & & & & & & & & & 0 & \\
\hline
\end{tabular}

Table A-42. Percent of Possible Daytime Data Passing SERI QC and Shadowband Alignment Tests

\begin{tabular}{|r|rrrrrrrrrrrr|}
\hline Year & Jan & Feb & Mar & Apr & May & June & July & Aug & Sept & Oct & Nov & Dec \\
\hline 85 & & & & & & & & 4 & 93 & 75 & 82 & 94 \\
86 & 96 & 88 & 92 & 99 & 96 & 87 & 67 & 87 & 92 & 72 & 76 & 93 \\
87 & 85 & 62 & 72 & 99 & 95 & 94 & 98 & 97 & 98 & 98 & 97 & 98 \\
88 & 86 & 85 & 89 & 88 & 57 & 0 & 0 & 0 & 0 & 0 & 14 & 99 \\
89 & 100 & 91 & 83 & 93 & 97 & 93 & 77 & 70 & 75 & 75 & 97 & 91 \\
90 & 84 & 74 & 78 & 92 & 90 & 97 & 90 & 85 & 84 & 90 & 95 & 81 \\
91 & 89 & 87 & 80 & 78 & 79 & 85 & 90 & 82 & 92 & 90 & 79 & 94 \\
92 & 84 & 70 & 68 & 77 & 78 & 72 & 54 & 94 & 98 & 96 & 87 & 99 \\
93 & 87 & 86 & 91 & 97 & 87 & 86 & 97 & 98 & 98 & 94 & 94 & 0 \\
& & & & & & & & & & & & \\
\hline
\end{tabular}


Public reporting burden for this collection of information is estimated to average 1 hour per response, including the time for reviewing instructions. searching existing data

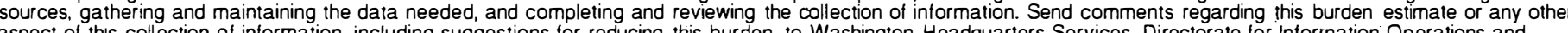
aspect of this collection of information, including suggestions for reducing this burden, to Washington Headquarters Services, Directorate for Inforrnation Operations and Reports, 1215 Jefferson Davis Highway, Suite 1204, Arlington, VA 22202-4302. and to the Office of Management and Budget, Paperwork Reduction Project (0704-0188), Washington, DC 20503
1. AGENCY USE ONLY (Leave blank)
2. REPORT DATE.
August 1994

\section{REPORT TYPE AND DATES COVERED}
technical report

4. TITLE AND SUBTITLE

Summary Information and Data Sets for the HBCU Solar Measurements Network

5. FUNDING NUMBERS

RA411040

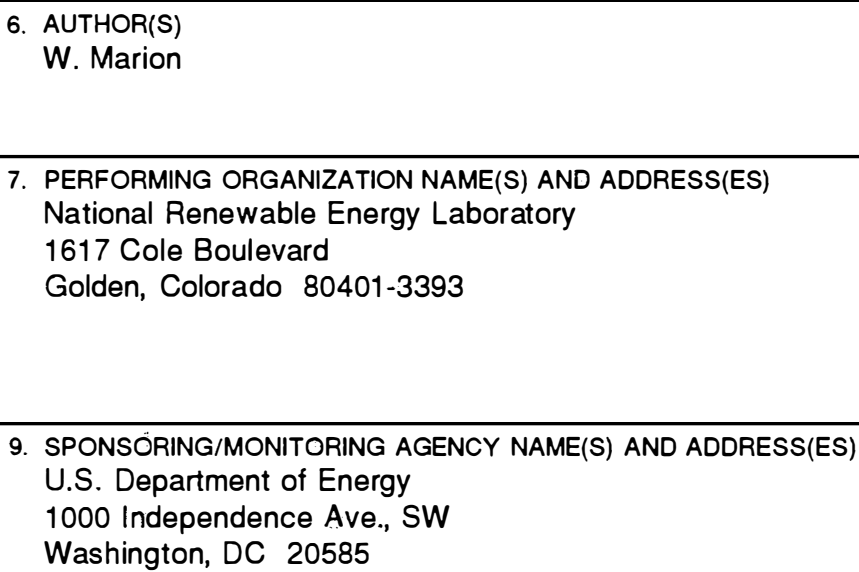

9. SPONSORING/MONITORING AGENCY NAME(S) AND ADDRESS(ES)

U.S. Department of Energy

1000 Independence Ave., SW

Washington, DC 20585

8. PERFORMING ORGANIZATION REPORT NUMBER TP-463-7090

10. SPONSORING/MONITORING

11. SUPPLEMENTARY NOTES

12a. DISTRIBUTION/AVAILABILITY STATEMENT National Technical Information Service U.S. Department of Commerce

5285 Port Royal Road

Springfield, VA 22161

\section{AGENCY REPORT NUMBER}

13. ABSTRACT (Maximum 200 words)

This report provides summary information and describes hourly data sets for solar radiation elements, such as global horizontal and diffuse horizontal radiation, measured from 1985 to 1993 in the southeastern United States. The measurements were made by the Historically Black Colleges and Universities (HBCU) Solar Measurements Network, which is maintained and operated by faculty and students from the six participating colleges and universities and managed by the National Renewable Energy Laboratory for the U.S. Department of Energy. The report describes the HBCU stations, the measurement equipment, and the data collection, processing, and quality assessment procedures. It also explains the positions of the solar radiation elements within the hourly data sets. Longterm averages and monthly variability for the solar radiation elements are presented in tables. The appendix contains information about when instruments and calibration factors were changed and also provides quality assessment summaries for daylight hours for each month of record.

\begin{tabular}{|c|c|c|c|c|}
\hline \multirow[t]{2}{*}{14.} & \multirow{2}{*}{\multicolumn{3}{|c|}{$\begin{array}{l}\text { SUBJECT TERMS } \\
\text { solar measurements; solar resource assessment; solar radiation; global horizontal radiation; } \\
\text { diffuse horizontal radiation; Historically Black Colleges and Universities (HBCUs) }\end{array}$}} & \multirow[t]{2}{*}{$\begin{array}{c}\text { 15. NUMBER OF PAGES } \\
68\end{array}$} \\
\hline & & & & \\
\hline 7. & $\begin{array}{l}\text { SECURITY CL } \\
\text { OF REPORT } \\
\text { unclassified }\end{array}$ & $\begin{array}{l}\text { 18. SECURITY CLASSIFICATION } \\
\text { OF THIS PAGE } \\
\text { unclassified }\end{array}$ & $\begin{array}{l}\text { 19. SECURITY CLASSIFICATION } \\
\text { OF ABSTRACT } \\
\text { unclassified }\end{array}$ & $\begin{array}{l}\text { 20. LIMITATION OF ABSTRACT } \\
\text { UL }\end{array}$ \\
\hline
\end{tabular}

